\title{
Differential Roles of Tri10 and Tri6 in Fusarium graminearum
}

\author{
By: Winnie Wai Yan Leung \\ B.Sc.H (University of Toronto, ON Canada)
}

A dissertation submitted to the Faculty of Graduate Studies and Research of Carleton University, in partial fulfillment of the requirements of the degree of Master of Science in Biology

Carleton University

Ottawa, ON Canada

(C) Winnie Leung, 2009 


\begin{abstract}
Fusarium graminearum is a globally distributed fungal pathogen that causes
\end{abstract} disease in cereal crops such as wheat and barley and it also contaminates host plants with a secreted mycotoxin known as deoxynivalenol. Consumption of infected or contaminated cereal products is hazardous to animals, therefore, fungal infection and contamination cause major economic losses in agriculture industries worldwide. My thesis project focused on characterizing two positive regulators, Tri10 and Tri6, of the trichothecene biosynthesis pathway in F. graminearum. Gene expression profiling of Tril04 and Tri64 mutant strains under DON-inducing conditions provided evidence that the regulatory roles of the two studied genes extend beyond the trichothecene biosynthesis pathway. They are potential regulators of another clustered secondary metabolic pathway, butenolide synthesis pathway, and also genes involved in primary metabolism. In contrast to a prior regulatory model of the trichothecene biosynthesis pathway, RNA transcript analysis demonstrated that regulation of Tril0 and Tri6 gene expressions are independent of each other. The distinct gene sets regulated by Tril0 and Tri6 indicate different, albeit overlapping, roles in regulating gene expression. 


\section{Acknowledgements}

First of all, I would like to sincerely thank my supervisor, mentor and friend Dr. Gopal Subramaniam for his guidance and support for the past two years. Not only has he trained me to become a better scientist, to always do things the 'right' way, but has also equipped me with tools and methodology to overcome future obstacles, science-related or not.

I would like to express my gratitude to my co-supervisor Dr. Owen Rowland, for his patience, understanding, and for always taking time out of his busy schedule to answer my questions. I would also like to extend my gratitude to Dr. Therese Ouellet, who has provided me with invaluable input and ideas for the past two years as well.

Without a harmonious working environment, I would have never accomplished what I have for my thesis. For this I must thank my lab members, in alphabetical order, Tina Kirkam, Charles Nasmith, Maria Villota, Sean Walkowiak and Li Wang. Thank you for tolerating my weaknesses, and also for cheering me up on a bad day. This experience would not have been the same without any of you.

Last, but most importantly, I would like to thank my parents, Horace and Josephine Leung, for always being there and providing your support for my decisions. I hope I do you proud. Also, I would like to thank my sister Kitty Ka Yan Leung, whose humour always makes me laugh and brighten my day.

"Courage does not always roar. Sometimes courage is the little voice at the end of the day saying I will try again tomorrow." - Maryanne Radanbacher 


\section{Table of Contents}

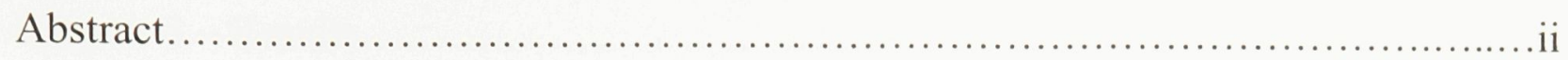

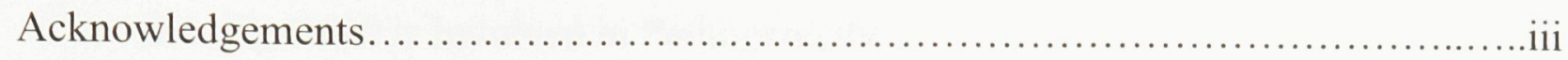

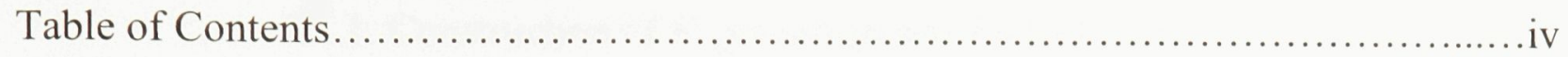

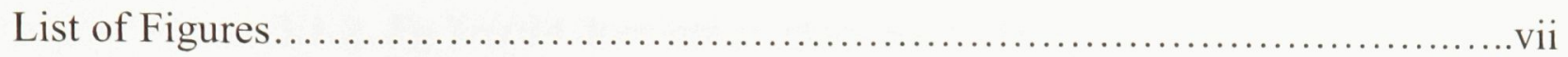

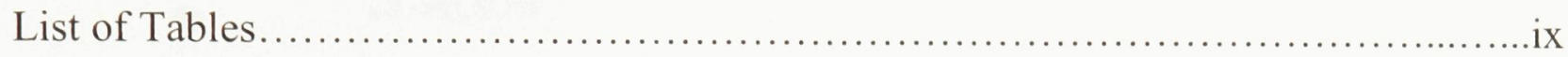

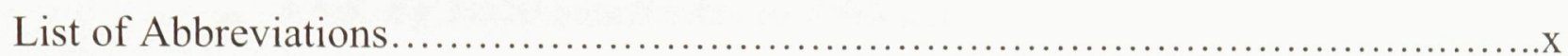

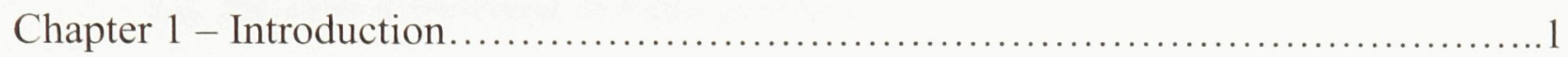

1.1. Fusarium species: An Overview...................................

1.1.1. Diseases caused by Fusarium species..........................1

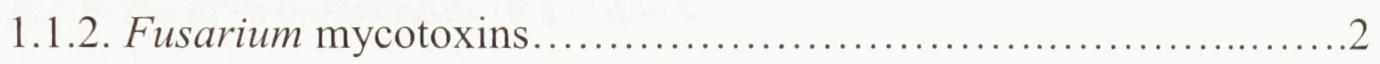

1.1.3. Economic impacts of Fusarium infection...................... 9

1.2. Fusarium graminearum and Host Range............................... 11

1.3. Life Cycle of Fusarium graminearum ................................ 13

1.4. The Role of Trichothecenes in Fusarium Pathogenicity....................... 16

1.5. Trichothecene Biosynthesis Pathway................................. 19

1.6. Regulatory Genes of the Trichothecene Biosynthesis Pathway: Tril0 and

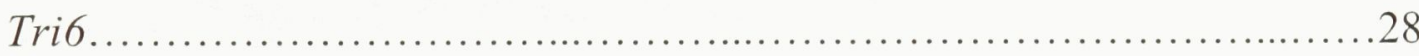

1.7. Thesis Objectives................................................

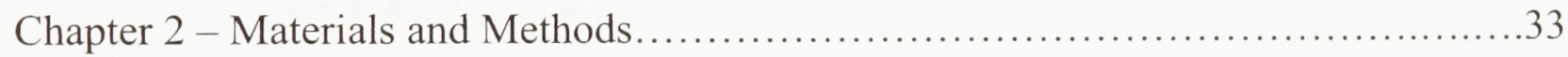

2.1 Fusarium Strains, Media and Culture Conditions.......................... 33

2.2 Gene Disruption Constructs, Protoplast Preparation and Fungal

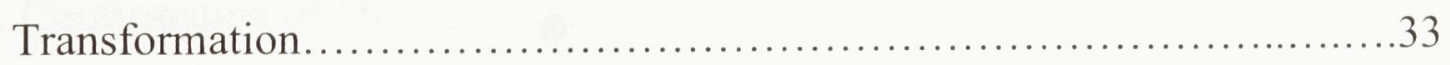

2.3 Complementation of Tri10 and Tri6 Deleted Mutant Strains ....................34

2.4 DNA Isolation and Analysis............................................ 35

2.5 RNA Extraction and Microarray Analysis................................... 36

2.6 Northern Analysis and Hybridization of Radiolabeled Probes.................39

2.7 Wheat Infection Assays........................................ 41 
Chapter 3 - Results

3.1. Fg Tri10 is Involved in Pathogenicity..................................43

3.1.1. Construction of $F$. graminearum Tril04 mutant strain............43

3.1.2. Fg Tril04 does not produce trichothecene derivative

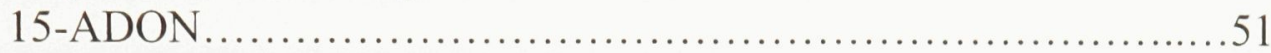

3.1.3. Fg Tril0 contributes to virulence............................56

3.2. Fg Tri6 is Involved in Pathogenicity...................................59

3.2.1. Disruption of Fg Tri6 using Homologous Recombination............59

3.2.2. Fg Tri6 is required for trichothecene production..................63

3.2.3. Fg Tri6 contributes to virulence.............................66

3.3. Establishment of a Relationship between Tri10 and Tri6 in vitro............69

3.3.1. Profiling of genes regulated in Tri104 and Tri64 under

DON-inducing conditions in vitro..............................69

3.3.2. Fg Trilo and Tri6 co-regulate genes involved in secondary metabolism....................................... 73

3.3.2.1. Fg Tri10 and Tri6 regulate the Tri gene cluster...........74

3.3.2.2. Fg TrilO and Fg Tri6 regulate the butenolide pathway in vitro....

3.3.3. Fg Trilo and Fg Tri6 regulate cytochrome $\mathrm{P} 450$ oxygenases in vitro....

3.3.4. Differential regulation of genes by Trilo and Tri6 .84

3.4. Confirmation of Microarray Data by RNA Blot Analysis of Selected Genes. .86 


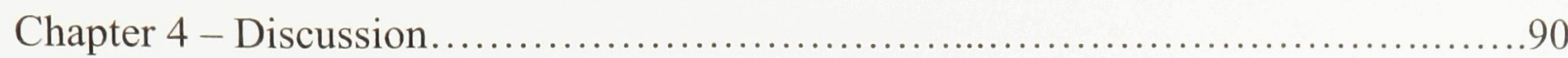

4.1. Coordinated and non-coordinated expression of Tri genes by

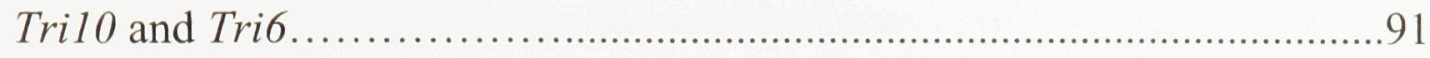

4.2. Comparison of in vitro and in planta gene expression profiles..............93

4.3. Roles of Tri10 and Tri6 in secondary metabolism......................95

4.4. Other candidates of the trichothecene biosynthesis pathway...............97

4.5. Differential regulation of genes by Tril0 and Tri6 ....................98

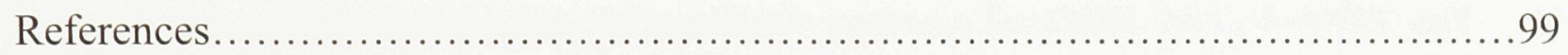

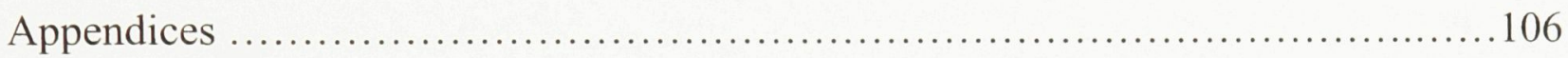




\section{List of Figures}

Figure 1: Chemical structures of major type B trichothecenes......................... 7

Figure 2: Tri gene cluster in T2-toxin-producing F. sporotrichioides strain, NIVproducing $F$. graminearum strain and DON-producing $F$. graminearum strain. .21

Figure 3: Proposed trichothecene biosynthesis pathway for DON, NIV, 4-ANIV and $15-\mathrm{ADON}$ .26

Figure 4: Generation of Tri10 disruption construct 45

Figure 5: Confirmation of Trilo disruption and complementation in $F$. graminearum .48

Figure 6: DNA blot analysis of Tri10 $\Delta$ and Tri6 $\Delta$ mutant strains.

Figure 7: HPLC chromatograms of 15-ADON standard

Figure 8: HPLC chromatograms of $F$. graminearum Tri10 0 and Tril0 $\Delta-A B$ strains cultured under DON-inducing conditions 55

Figure 9: Fg Tri10 contributes to virulence in F.graminearum 58

Figure 10: Confirmation of Tri6 disruption and complementation in $F$. graminearum.

Figure 11: HPLC chromatograms $F$. graminearum Tri6 6 and Tri6 $6-A B$ strains cultured under DON-inducing conditions 
Figure 12: Fg Tri6 contributes to virulence in $F$. graminearum......................68

Figure 13: Venn diagram indicating the number of genes commonly and differentially regulated in WT, Fg Tri64, and Fg Tr104

Figure 14: Potential action of a cytochrome P450 oxygenase in the trichothecene biosynthesis pathway....

Figure 15: Gene expressions of Tri1, fg00007, Tril0 and Tri6 under non DONinducing and DON-inducing conditions..... 


\section{$\underline{\text { List of Tables }}$}

Table 1: Functional classification of fifteen Tri genes in Fusarium graminearum.

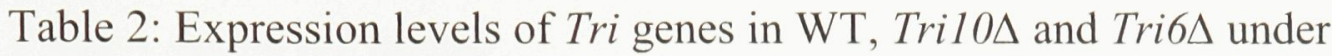
DON-inducing conditions. .76

Table 3: Expression levels of genes involved in the butenolide biosynthetic pathway under DON-inducing conditions. 78

Table 4: Expression levels of genes encoding for cytochrome P450 oxygenases regulated by Tril 10 and Tri6 under DON-inducing conditions....

Table 5: MIPS functional category of genes potentially activated by Tri 10 or Tri6 under DON-inducing conditions 


\section{List of Abbreviations}

$\mathrm{ATA}=$ alimentary toxic aleukia

$\mathrm{bp}=$ base pairs

$\mathrm{CPM}=$ counts per minute

Cy-3 = cyanine 3

Cy-5 = cyanine 5

DMSO = dimethyl sulfoxide

$\mathrm{DON}=$ deoxynivalenol

$\mathrm{EST}=$ expressed sequence tags

$F g=$ Fusarium graminearum

FHB $=$ Fusarium head blight

Fs $=$ Fusarium sporotrichioides

$\mathrm{g}=$ gram

HPLC $=$ high performance liquid chromatography or high pressure liquid chromatography

hrs $=$ hours

Hyg = hygromycin

$\mathrm{Kb}=$ kilo basepair

$\mathrm{mAU}=$ milli-absorbance

$\mathrm{Mb}=$ mega base pairs

$\mathrm{mg}=$ milligram

$\mathrm{mL}=$ millilitre

$\mathrm{ng}=$ nanogram 


$$
\begin{aligned}
& \text { NIV = nivalenol } \\
& \text { RPM = revolutions per minute } \\
& \text { PCR = polymerase chain reaction } \\
& \text { PEG = polyethylene glycol } \\
& \text { WT = wild type } \\
& \mathrm{ZEA}=\text { Zearalenone } \\
& 4-\mathrm{ANIV}=4 \text {-acetylnivalenol } \\
& 15 \text {-ADON = 15-acetyldeoxynivalenol } \\
& \mu \mathrm{g}=\text { microgram } \\
& { }^{\circ} \mathrm{C}=\text { degree Celsius }
\end{aligned}
$$




\section{Chapter 1 - Introduction}

\subsection{Fusarium species: An Overview}

\subsubsection{Diseases Caused by Fusarium species}

Fusarium species are globally distributed fungal pathogens that cause devastating diseases in agriculturally important cereal crops such as wheat and barley (Parry et al., 1995). The most commonly documented disease, Fusarium head blight (FHB), also known as scab, is characterized by bleaching, sometimes browning, of infected spikelets of wheat (Parry et al., 1995). In 1995, the five predominant species responsible for FHB around the world were $F$. culmonrum, $F$. avenaceum, $F$. poae, $F$. nivale, and $F$. graminearum. However, by year 2000, F. graminearum had become the primary causal agent of FHB (Parry, 1990; Windels, 2000). Other Fusarium-associated diseases include Fusarium foot rot, root rot and seedling blight (Parry et al., 1995, Couture et al., 2002; Khan et al., 2006). Symptoms of foot rot are typically described as browning at the stalk or stem base of the infected cereal host plant, and the causal species were identified to be Fusarium nivale, F. culmorum, F. avenaceum and F. graminearum (Parry, 1990).

Although both FHB and foot rot diseases are mainly caused by the same Fusarium species, the biological factors and abiotic conditions that determine the establishment of one disease over another are not well understood. Nonetheless, records show that FHB is more likely to occur in early spring during the anthesis stage of cereal host plants, whereas foot rot disease is more frequently observed in autumn on mature plants (Parry et al., 1995; Parry, 1990). Hence, the developmental stage of host plants might be a factor in determining the conquest of each disease. Unlike the wide range of causal species responsible for FHB and foot rot, root rot in crops is most often caused by 
Fusarium oxysporum (Sutherland, 1990; Michielse and Rep, 2009). Plants with root rot exhibit symptoms such as stunted growth, leaf discolouration from green to light green or yellow, and narrowed reddish-brown shoots (Sutherland, 1990). Finally, seedling blight occurs when Fusarium-infected seeds are sowed, or when seeds are planted in a Fusarium-contaminated field (Glynn et al., 2007). Depending on the severity of the disease, infected seeds may fail to germinate. However, if germination was successful, the plant development can be affected manifested by stunted shoot and root growth (Glynn et al., 2007). Many Fusarium species cause seedling blight, with the most prevalent species corresponding to the ones mentioned for FHB (Khan et al., 2006).

\subsubsection{Fusarium mycotoxins}

The capability of Fusarium spp. to cause disease is often attributed to their ability to produce and secrete mycotoxins. To date, it is known that these secondary metabolites affect plant or animal biological systems through various means such as interfering with DNA replication or protein synthesis, targeting host immune system and blocking of important metabolic pathways (Pitt, 2000, Desjardins and Proctor, 2007). Since mycotoxins are stable even under sterilizing and food-processing conditions, they also pose serious health hazards to humans and livestocks that have cereal-grain food products in their diets (Yazar and Omurtag, 2008). Based on their frequent occurrence in nature and high toxicity to humans and animals, Fusarium mycotoxins have been classified into three major groups: zearalenones, fumonisins, and trichothecenes (De Nijs et al., 1996, Desjardins, 2006). Of the three toxins, zearalenones appear to be the least toxic as they have not been associated with any fatal illnesses after consumption, whereas 
trichothecenes and fumonisins were reported to have caused deaths in humans and animals (Desjardins and Proctor, 2007, Yazar and Omurtag, 2008).

The biggest health concern regarding zearalenone is that it functionally mimics the hormone estrogen, and is able to bind and activate human and mammalian $\alpha$ - and $\beta$ estrogen receptors (Kuiper-Goodman et al., 1987). Even though this myco-estrogen is not currently linked to any human diseases, numerous swine disease outbreaks revealed that zearalenone is responsible for causing estrogenic syndrome in animals. General symptoms of the estrogenic syndrome in females include enlargement of the mammary gland and reproductive organs such as the uterus and fallopian tubes, decreased number of viable offspring, decreased weight and size of offspring and in more serious cases, ovary deterioration that leads to infertility (Kordic et al., 1992, Desjardins, 2006). Affected males exhibit similar symptoms, including the enlargement of their mammary gland, as well as deterioration of their version of primary reproductive organ, the testes (Kordic et al., 1992, Desjardins, 2006). At present, there are no indications of zearalenones being toxic to plants. Zearalenones (ZEA), even at high concentrations $(310 \mu \mathrm{M})$, did not affect growth of wheat seedlings (Desjardins, 2006). It has been detected in corn and other crops of Australia, Asia, Europe, Africa, South America and North America (Kuiper-Goodman et al., 1987). Several studies have established that ZEA is a heat stable metabolite, as incubation of zearalenone or ZEA-contaminated corn samples at a temperate range of $120^{\circ} \mathrm{C}$ to $150^{\circ} \mathrm{C}$ for four to forty-four hours did not reduce the levels of the mycotoxin (Bennett et al., 1980, Kuiper-Goodman et al., 1987). Due to its thermal stability, natural accumulation on crops and harmful effects on farm 
animals, 16 countries now limit the amount of zearalenone to less than $1000 \mu \mathrm{g}$ per $\mathrm{kg}$ in corn and other crops (ARO, 2003). Strains of Fusarium that consistently produce zearalenone in maize or other crops belong to the species $F$. graminearum, $F$. crookwellense, F. culmorum, F. equiseti, and F. semitectum (Desjardins, 2006).

All fumonisins share a backbone of 20 -carbon aliphatic straight chain, with two ester-linked side-chains at carbon 14 and carbon 15 (Pitt, 2000). The principal fumonisins found naturally in crops are the three homologues in series B, designated $\mathrm{FB}_{1}, \mathrm{FB}_{2}$ and $\mathrm{FB}_{3}$ (Yazar and Omurtag, 2008). These three homologues differ by the presence of a hydroxyl group on carbon 5 or carbon $10 . F$. verticillioides, F. proliferatum and one strain of F. oxysporum have been found to produce fumonisins, with production of each fumonisin homologue being strain-specific as opposed to species-specific (Desjardins, 2006). This group of mycotoxin has been classified by the International Agency for Research on Cancer (IARC) as group 2B, which represents the "probably carcinogenic" group (IARC, 1993). This decision was supported by the unusually high incidence of esophageal cancer in South Africa from 1955 to 1990, which corresponds to the time when collected maize samples, their major food source, were heavily contaminated with $\mathrm{FB}_{1}$ and $\mathrm{FB}_{2}$ (Desjardins, 2006). A positive correlation between high levels of fumonisins and esophageal cancer was also observed in rural parts of China. Fumonisins at a concentration ranging from 1 to $50 \mu \mathrm{M}$ can inhibit the root and shoot growth of maize and tomato seedlings (Desjardins, 2006). Spraying $\mathrm{FB}_{1}$ at a concentration of up to $200 \mu \mathrm{g}$ per $\mathrm{mL}$ on adult weed and crop plants such as cucumber and soybean plants induced necrosis and wilting, yet there are no visible effects when 
$\mathrm{FB}_{1}$ was sprayed on monocots such as wheat and barley (Abbas and Boyette, 1992). The molecular effects of fumonisins on plants remain obscure. $\mathrm{FB}_{1}$ was first identified in the 1980s and by 2003 six countries had limited the amount of fumonisins on crops to less than 3000 ug per $\mathrm{kg}$ (ARO, 2003). Like zearalenones, fumonisins can be found in all major continents and are stable at temperatures as high as $150^{\circ} \mathrm{C}$ (Desjardins, 2006, Yazar and Omurtag, 2008).

With over 200 trichothecenes discovered, this group of mycotoxin is classified into four types, A to D, according to their chemical structure (Desjardins, 2006).

Trichothecenes are a group of sesquiterpenoid compounds with a general backbone of 12, 13-epoxytrichothec-9-enes, with a double bond between carbon 9 and carbon 10 , and an epoxide ring linking carbon 12 and carbon 13 (Desjardins, 2006). All Fusariumproduced trichothecenes belong to either type A or type B, with T-2 toxin being the most prominent type A trichothecene, and the most frequently encountered type B trichothecenes in crops are nivalenol (NIV) and deoxynivalenol (DON) (Desjardins, 2006, Kimura et al., 2007). Type A trichothecenes are defined by the absence of a ketone group on carbon 8 , and within this type, they vary from the side-chains added at carbon 3, 4, 8 and 15 (Kimura et al., 2007). Alternatively, type B trichothecenes are distinguished by the presence of a ketone at carbon 8 , allowing for side-chain variation at carbon 3, 4, 7 and 15, see Figure 1 (Kimura et al., 2007). 
Figure 1: Chemical structures of type B trichothecenes.

A) General backbone structure of type B trichothecenes, described as 12, 13epoxytrichothec-9-en-8-one. (Image obtained from Kimura et al., 2007). Carbons 12 and 13 are linked by an epoxide ring whereas carbons 8 and 9 share a double bond. Red square indicates a ketone at carbon 8 , a distinguishing feature of type B trichothecenes. Chemotypes of type B trichothecenes differ from each other based on the R-groups attached to carbons $3,4,7$ and 15 .

B) Functional groups specific to DON, NIV, 15-ADON and 3-ADON at carbons 3, 4, 7 and 15 . R1 refers to carbon 3, R2 to carbon 4, R3 to carbon 15 and R4 to carbon 7. For example, DON is chemically described as 3, 7, 15-trihydroxy-12,13-epoxytrichothec-9en-8-one, illustrating the hydroxyl groups at carbons 3, 7 and 15 in addition to the general type B trichothecene backbone. (Kimura et al., 2007, Desjardins, 2006) 
A)

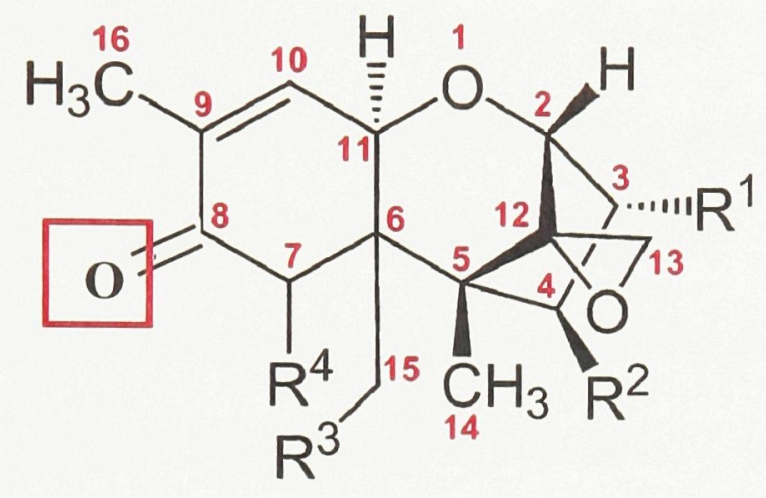

B)

\begin{tabular}{|l|c|c|c|c|}
\hline Type B Trichothecene & R1 & R2 & R3 & R4 \\
\hline Deoxynivalenol (DON) & $-\mathrm{OH}$ & $-\mathrm{H}$ & $-\mathrm{OH}$ & $-\mathrm{OH}$ \\
\hline Nivalenol (NIV) & $-\mathrm{OH}$ & $-\mathrm{OH}$ & $-\mathrm{OH}$ & $-\mathrm{OH}$ \\
\hline 15-acetyl-deoxynivalenol (15-ADON) & $-\mathrm{OH}$ & $-\mathrm{H}$ & $-\mathrm{OAc}$ & $-\mathrm{OH}$ \\
\hline 3-Acetyldeoxynivalenol (3-ADON) & $-\mathrm{OAc}$ & $-\mathrm{H}$ & $-\mathrm{OH}$ & $-\mathrm{OH}$ \\
\hline
\end{tabular}


Type $\mathrm{C}$ trichothecenes consist of an extra epoxide ring linking carbons 7 and 8 , whereas type D trichothecenes are differentiated by the 4, 15-macrocyclic ring (Kimura et al., 2007). Trichothecenes are not only produced by Fusarium species, they are also produced by other fungal genera such as Trichoderma, Trichothecium, Stachybotrys and Myrothecium (Desjardins, 2006). Nivalenol harbours hydroxyl groups at all four carbons available for side-chains whereas deoxynivalenol only harbours three hydroxyl groups, with carbon 4 unattached (Figure 1). T-2 toxin is produced by $F$. sporotrichioides, $F$. poae, F. equiseti and F. acuminatum (Yazar and Omurtag, 2008). DON is primarily produced by $F$. graminearum and $F$. culmorum, which are two of the five major species responsible for causing FHB (Yazar and Omurtag, 2008, Windels, 2000). Some isolates of these two Fusarium species can also produce NIV, but the main producers of this toxin are F. cerealis and F. poae (Yazar and Omurtag, 2008). International Agency for Research on Cancer (IARC) classified this group of Fusarium mycotoxins as group 3, indicating their carcinogenicity in humans was not classifiable due to insufficient and limited evidence (IARC, 1993). The three representative trichothecenes have demonstrated their high toxicity in humans and animals through previous outbreaks and clinical studies. In humans, T-2 toxin causes a condition known as "alimentary toxic aleukia" (ATA), where affected individuals mainly suffer from a reduction in white blood cells due to damaged bone marrow, which leads to immunosuppression, sometimes resulting in death. DON is sometimes referred to as vomitoxin, because it induces vomiting in animals, including humans, upon consumption of contaminated grain. In 1933, an outbreak of red mold disease, termed akakabi-byo, was associated with DON and nivalenol contamination. Affected patients experienced vomiting, nausea, trembling 
and visual hallucinations. There were no reports of deaths. Although the fungus $F$. graminearum, which produces DON and NIV, was consistently isolated from the moldy grains associated with the outbreak, traces of DON and NIV were never reported (Desjardins, 2006). This might be due to the fact that DON was not isolated and characterized until the 1970s. The symptoms of DON were confirmed by feeding experiments of swine, where pigs suffered from vomiting after they were fed with grains contaminated with $0.15 \mathrm{mg}$ per kg of DON (Pestka and Smolinski, 2005). At concentrations as low as $1 \mu \mathrm{M}$ to $10 \mu \mathrm{M}$, trichothecenes can inhibit shoot and root growth in pea and lettuce seedlings. In contrast to zearalenones and fumonisins, trichothecenes have inhibitory effects on monocots such as wheat. T-2 toxin appears to be the most toxic trichothecene, inhibiting $50 \%$ of wheat seedling shoot growth at $2 \mu \mathrm{M}$, with DON at $3 \mu \mathrm{M}$ and NIV at $21 \mu \mathrm{M}$ (Shimada and Otani, 1990, Desjardins, 2006).

\subsubsection{Economic Impacts of Fusarium Infection}

In spite of the slightly higher phytotoxicity of T-2 toxin, DON is considered the most important trichothecene of the three because of its prevalence in cereal crops and its association with FHB. Incidence of DON was reported worldwide, whereas NIV was hardly detected in Africa, North America and South America and T-2 toxin was never reported in Africa and East Asia (Desjardins, 2006). A study performed in 2003 indicated the occurrence of DON is more frequent than that of NIV and T-2 toxin in Southern Ontario. For instance, $58 \%$ of harvested barley samples tested positive for DON, and the average concentration of contaminated samples was $260 \mathrm{ng}$ per g but none tested positive for NIV and T-2 toxin (Lombaert et al., 2003). 
Both Fusarium-associated disease and Fusarium mycotoxin contamination has the potential to reduce crop yield and grain quality. In the 1990s, FHB epidemics in North America cost farmers in Quebec and Ontario up to US \$220 million in crops while Manitoba farmers suffered a loss of approximately US $\$ 300$ million (Windels, 2000). During the same period, the United States lost up to US $\$ 2.7$ billion in wheat and barley due to Fusarium infection (Goswami et al., 2004). According to the Government of Alberta, Canada continues to experience scab epidemics, and suffers from a loss of about $\$ 100$ million annually in Manitoba alone. 


\subsection{Fusarium graminearum and Host Range}

F. graminearum consists of nine phylogenetically distinct subspecies: $F$. austroamericanum, F. meridionale, F. boothii, F. mesoamericanum, F. acaciaemearnsii, F. asiaticum, F. graminearum, F. cortaderiae, and F. brasilicum (O'Donnell et al., 2004). In addition to the molecular markers, morphological features such as conidial width and length, presence or absence of an apical conidial beak, morphological symmetry and the widest position of conidia are also used to distinguish these species (O’Donnell et al., 2004). Global sampling indicated that some of these species are geographically limited, while others are found in more than one continent. Since geography and climate dictate the type of crops grown in the area, these species have evolved and adapted the ability to cause disease in different hosts accordingly. Four of the nine species, F. cortaderiae, F. brasilicum, F. austroamericanum, and $F$. meridionale, belong to the South American clade, which corresponds to their geographic distribution (O'Donnell et al., 2000, O'Donnell et al., 2004). Each of these four species inhabit different hosts, with $F$. cortaderiae isolated from Cortaderia spp., a type of grass native to South America, F. brasilicum extracted from oats, and F. meridionale from corn (O'Donnell et al., 2000, O'Donnell et al., 2004). Strains of F. austroamericanum were recovered from three different hosts, including polypore mushrooms, corn and herbaceous vine, suggesting a diverse host range within the species (O'Donnell et al., 2000, O’Donnell et al., 2004). The Central American-distributed species $F$. mesoamericanum infects fruit crops, as strains of it were isolated from bananas and others from grape ivy. Strains belonging to the species F. acaciae-mearnsii were discovered in Australia inhabiting a native legumous tree known as Acacia mearnsii 
(O’Donnell et al., 2000, O’Donnell et al., 2004). F. asiaticum causes disease in barley, wheat and corn, and is restricted to Asia (O'Donnell et al., 2000, O'Donnell et al., 2004). The two species $F$. boothi and $F$. graminearum are perhaps the two most widespread species of all, with F. boothi found in Africa, Mexico and Central America, and $F$. graminearum found in all of the major continents (O'Donnell et al., 2000, O'Donnell et al., 2004). However, their hosts remain limited to cereal crops, with $F$. boothi mainly infecting corn and F. graminearum infecting wheat, barley and corn (O'Donnell et al., 2000, O’Donnell et al., 2004). 


\subsection{Life Cycle of Fusarium graminearum}

The homothallic ascomycetes, $F$. graminearum, can alternate between its anamorphic state (F. graminearum Schwabe) and teleomorphic state (Gibberella zeae Schwein) to produce asexual conidia and sexually derived ascospores respectively (Stack, 1989, Trail et al., 2002). While conidia are equally effective in causing disease, ascospores are considered the primary inoculum for FHB because perithecia, fruiting bodies from which ascospores are discharged, are more frequently found on infected cereal crops (Stack, 1989, Guenther and Trail, 2005). In winter, the fungus survives in the form of perithecia on crop debris, even though chlamydospores, a form of conidia, and hyphal fragments can also be found in the soil (Goswami and Kistler, 2004, Champeil et al., 2004). Both perithecia and chlamydospores have thick, multi-layered outer shells, allowing them to endure through the harsh winter months (Mehrotra and Aneja, 1990). Although undamaged, the low temperature, lack of sunlight and drought inhibited the development of these propagules, inducing a dormant phase (Champeil et al., 2004). In spring, an increase in daylight, temperature and rainfall offset previous effects and allowed for maturation of perithecia and chlamydospores to continue (Champeil et al., 2004). Since F. graminearum does not produce specialized, penetrating structures such as an appresorium, spore maturation and dispersal must be coordinated with anthesis, when the florets are most susceptible and provide easy access into the host plant. Inside a mature perithecium, ascospores are suspended in sac-like spore-producing cells known as asci, which will individually extend and protrude through the ostiole, a small hole at the top of the perithecium, to discharge ascospores (Mehrotra and Aneja, 1990). This release of ascospores is triggered by a period of drought followed by high 
humidity, and the optimal temperature ranges from $13^{\circ} \mathrm{C}$ to $22^{\circ} \mathrm{C}$ (Champeil et al., 2004). The mechanism behind ascospore discharge has not been determined, but it is speculated that the presence of water increases osmotic pressure built up by an accumulation of mannitol sugar and an influx of potassium ions inside the vacuole of an ascus, hence leading to a forceful ejaculation (Trail et al., 2002, Trail et al., 2005). Ascospores do not always land on a susceptible part of the host plant, and rely on wind and splash created by rain for aerial dispersal (Goswami and Kistler, 2004, Champeil et al., 2004). Following spore germination, the fungal hyphae enter into the flower, colonizing the anthers and ovaries, and move towards the glumes and the rachis. Colonization of the anthers stimulates hyphae growth, since these male reproductive organs contain two virulence enhancers, choline and betaine. Both compounds contain an amide group and are transported into the fungus, promoting hyphal extension (Robson et al., 1995). As the hyphae progress towards the rachis, they penetrate and degrade hindering cells, until they have successfully colonized the entire wheat grain. In a more detailed study, Guenther and Trail used a F. graminearum GFP-tagged strain to examine the process of fungal invasion (Guenther and Trail, 2005). It was discovered that this pathogen takes the form of haploid mycelia when moving down the rachis, but it converts into dikaryotic hyphae when colonizing chlorenchyma cells and stomates (Guenther and Trail, 2005). Dikaryotic hyphae refer to fungal cells that harbour two nuclei, which occurs during sexual reproduction. This change in reproductive state is consistent with the emergence of sexually-developed perithecia within chlorenchyma tissue and stomates, where dikaryotic hyphae are found (Guenther and Trail, 2005). These perithecia will overwinter in crop debris and become the primary inoculum for FHB the subsequent year. 
Microarray analysis revealed that ion transporters play an important role in perithecium development (Hallen et al., 2007). In particular, potassium, chloride and calcium ion channels are of interest, because they were activated 72 to 96 hours after the onset of perithecium maturation, corresponding to the developmental stage where asci are formed (Hallen et al., 2007). These ion channels might play a role in ascospore discharge by allowing an influx of ions into the vacuoles of asci, ultimately leading to ascospore discharge. 


\subsection{The Role of Trichothecenes in Fusarium Pathogenicity}

Trichothecenes are typically described as virulence factors that contribute to pathogenicity of Fusarium spp. on cereal host plants. This is supported by the observation that a non DON-producing $F$. graminearum mutant strain, designated $F g$ Tri64 was able to infect the wheat cultivar Norm, but failed to spread beyond the site of infection (Seong et al., 2009). Independent of the pathogen, pure trichothecenes are also toxic to plants, as discussed in section 1.1.2. Studies have shown that the three trichothecenes, DON, NIV and T-2 toxin, inhibit protein synthesis by targeting the $23 \mathrm{~S}$ rRNA component of the ribosome that has peptidyl transferase activity (Nissen et. al., 2000). This transferase is responsible for forming peptide links between amino acids and tRNA in the process of translation (Nissen et. al., 2000). Inhibition of an important cellular process such as this generates a wide range of effects on the host plant and among them is the failure to translate genes involved in disease resistance, enabling the fungus to prevail in this host-pathogen battle. Some plants have evolved resistance to Fusarium infection, particularly against the effects of trichothecenes. The degree of resistance to FHB varies from cultivar to cultivar in cereal crops, but in general, there are five types of resistance. Type I resistance counteracts initial Fusarium infection (Schroeder and Christensen, 1963). Since infection requires the dispersal of ascospores and conidia onto susceptible plant organs, a cultivar with type I resistance possesses physical characteristics such as longer peduncle or stem that prevents spores from landing onto the flowers (Champeil et al., 2004). Also, cultivars with type I resistance decrease susceptibility to Fusarium infection by secreting anti-fungal compounds such as chitinases, which are fungal cell-wall degrading enzymes (Champeil et al., 2004). Type 
II resistance prevents the spread of the pathogen inside the host plant (Schroeder and Christensen, 1963). Resistant host plants do so by depositing callose and lignin into the periplasmic space to create a defense wall between fungal-colonized tissue and uninfected tissue, blocking further entry of the invading fungus and trichothecenes (Schroeder and Christensen, 1963, Champeil et al., 2004). In order for F. graminearum to overcome type II resistance, diffusion of DON into the host plant must precede the establishment of a defense wall, because inhibitory effects of this metabolite on protein synthesis can stop the production of callose and lignin (Champeil et al., 2004). Type III resistance refers to the resistance specifically against kernel infection utilizing similar morphological structures described for type I resistance (Foroud and Eudes, 2009). Host plants with type IV resistance have a modified peptidyl transferase that is not recognized by trichothecenes; therefore, mycotoxins have no affect on the plant's ability to synthesize callose and lignin, thus allowing the host to suppress fungal advancement through type II resistance (Champeil et al., 2004, Foroud and Eudes, 2009). Finally, type $\mathrm{V}$ resistance is the resistance against trichothecene accumulation (Champeil et al., 2004, Foroud and Eudes, 2009). Host plants demonstrating this type of resistance are divided into two classes: class 1 refers to plants that can chemically modify trichothecenes such that they render it non-toxic (Boutigny et al., 2008). For instance, certain FHB resistant host plants such as wheat cultivar Frontana have the ability to add a glucose molecule to the hydroxyl group at carbon-3 of type B trichothecenes, which greatly reduces their ability to inhibit protein synthesis (Miller and Arnison, 1986). Using an in vitro translation assay with firefly luciferase as a reporter marker, it was determined that $1 \mu \mathrm{M}$ of unglycosylaed DON inhibited translation by $36.8 \%$, while $20 \mu \mathrm{M}$ of DON-3-O- 
glucoside, glycosylated DON, inhibited luciferase translation by only $8 \%$ (Poppenberger et al., 2003). In Arabidopsis thaliana, glycosylation of DON is catalyzed by the enzyme deoxynivalenol-glucosyl-transferase (DOGT1), which codes for an UDPglucoysyltransferase (Poppenberger et al., 2003). However, the gene encoding DONglucosyltransferase in wheat has not been identified. Alternatively, class 2 host plants reduce trichothecene accumulation by inhibiting trichothecene biosynthesis in the fungal pathogen (Boutigny et al., 2008). It had been reported that flavonoids and furanocoumarins could inhibit $\mathrm{T} 2$-toxin production in $F$. sporotrichioides by more than $85 \%$ (Desjardins et al., 1988). Flavone was the only flavonoid tested that could inhibit trichothecene production, whereas examples of furanocoumarins that act as inhibitors include pimpinellin, xanthotoxin and xanthotoxol (Desjardins et al., 1988). The first compound formed in the trichothecene biosynthesis pathway, trichodiene, was detected in cultures, indicating these inhibiting compounds block the pathway in subsequent steps. 


\subsection{Trichothecene Biosynthesis Pathway}

The genome of Fusarium graminearum isolate PH-1 has been sequenced to 99.7\% completion and was released to the public by the Broad Institute in 2003 (http://www.broadinstitute.org/annotation/genome/fusarium_graminearum/MultiHome.ht $\mathrm{ml}) . F$. graminearum contains four chromosomes, with a genome size of $36.1 \mathrm{Mb}$ and encodes for approximately 13,937 predicted open reading frames (Goswami and Kistler, 2004, Trail, 2009). Most trichothecene genes involved in the biosynthesis pathway have been identified, with a majority of them found within the $25 \mathrm{~kb}$ core Tri cluster on chromosome 2 (Kimura et al., 2003). Comparison of the Tri cluster revealed allelic polymorphisms between chemotype-specific $F$. graminearum strains, and also between type A and type B trichothecene-producing Fusarium species (Brown et al., 2002, Kimura et al., 2003, Goswami and Kistler, 2004). Figure 2 illustrates that a functional Tri7 and Tril3 are present in the Tri cluster of both T-2 toxin-producing F. sporotrichioides strains and NIV-producing F. graminearum strains, but encodes for non-functional gene products in DON-producing $F$. graminearum strains. 
Figure 2: Tri gene cluster in T2-toxin-producing F. sporotrichioides strain, NIVproducing $F$. graminearum strain and DON-producing $F$. graminearum strain. (Brown et al., 2002)

The twelve Tri genes from the Tri gene cluster are represented by arrows, which indicate the direction of transcription. The number underneath each arrow identifies the Tri gene. F. sporotrichioides and NIV-producing $F$. graminearum strains encode functional Tri7 and Tri13 proteins, but DON-producing $F$. graminearum strain does not, as illustrated by the elimination of the corresponding genes. 
F. sporotrichioides

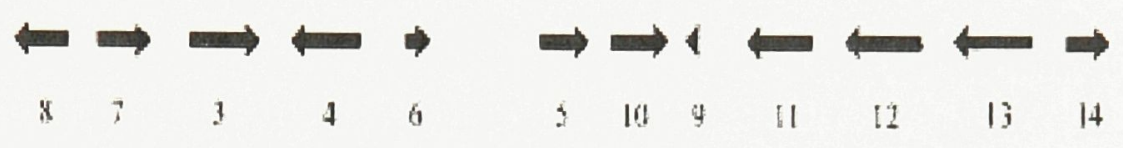

F. graminearum (NIV)

$\Leftrightarrow \Rightarrow \Rightarrow$

$\Rightarrow m 1 \Longleftrightarrow$

F. graminearum (DON)

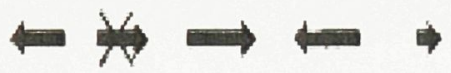

$\Rightarrow=1+\frac{1}{2} \Rightarrow$ 
The presence of Tri7 and Tril3 correlates specifically with the production of T2-toxin and NIV. Tri13 encodes a monooxygenase which hydroxylates carbon 4 of NIV and T-2 toxin while the protein product of Tri7 acetylates the oxygen atom attached to C4 of T-2 toxin, resulting in an acetyl-ester group in the final product (Lee et al., 2002). The hydroxyl group on $\mathrm{C} 4$ of nivalenol remains unaltered in the NIV-producing $F$. graminearum strain, Tri7 participates later on in the pathway to produce the NIV-related trichothecene, 4-acetylnivalenol, in 4-ANIV-producing $F$. graminearum strains (Lee et al., 2002, Brown et al., 2002). Factors regulating the functionality of Tri7 protein in NIV-producing and 4-ANIV-producing strains are not known.

To date, fifteen functional Tri genes have been identified in $F$. graminearum. Besides the twelve Tri genes in the core Tri cluster as indicated in Figure 1, there are also Tril on chromosome 1 (McCormick et al., 2004), Tri101 on chromosome 4 (Kimura et al., 1998) and Tril5 on chromosome 3 (Desjardins, 2006). Not all of these genes are involved in catalyzation of substrates; some are involved in regulation of the pathway while others are involved in transport (Kimura et al., 1998, Desjardins, 2006). These genes are functionally categorized into five groups, as shown in Table 1. 
Table 1: Functional classification of fifteen Tri genes in Fusarium graminearum

\begin{tabular}{|c|c|c|c|}
\hline Type of Function & Gene & $\begin{array}{c}\text { Specific Role in } \\
\text { Trichothecene Biosynthesis } \\
\text { Pathway }\end{array}$ & $\begin{array}{l}\text { Strains of } F \text {. } \\
\text { graminearum }\end{array}$ \\
\hline Sesquiterpene cyclase & Tri5 & $\begin{array}{c}\text { Cyclize farnesyl } \\
\text { pyrophosphate (initial } \\
\text { substrate) into trichodiene }\end{array}$ & DON, NIV \\
\hline \multirow[t]{4}{*}{ Cytochrome P450 } & Tri4 & $\begin{array}{l}\text { Hydroxylase (multiple } \\
\text { carbons) }\end{array}$ & DON, NIV \\
\hline & Trill & C15 hydroxylase & DON, NIV \\
\hline & Tril & C8 hydroxylase & DON, NIV \\
\hline & Tril3 & C4 hydroxylase & NIV \\
\hline \multirow{4}{*}{$\begin{array}{l}\text { Acylation/deacylation } \\
\text { (adding or removing } \\
\text { an acyl group) }\end{array}$} & Tri3 & C15-acetyltransferase & DON, NIV \\
\hline & Tril01 & C3-acetyltransferase & DON, NIV \\
\hline & Tri8 & C3 esterase & DON, NIV \\
\hline & Tri7 & C4-acetyltransferase & NIV \\
\hline \multirow[t]{3}{*}{ Regulation } & Tri6 & $\begin{array}{l}\text { Positive regulator } \\
\text { (transcription) }\end{array}$ & DON, NIV \\
\hline & Trilo & Positive regulator & DON, NIV \\
\hline & Tril5 & Negative regulator & DON, NIV \\
\hline Transport & Tril2 & $\begin{array}{l}\text { Transporter involved in } \\
\text { trichothecene efflux }\end{array}$ & DON, NIV \\
\hline \multirow[t]{2}{*}{ Unknown } & Tri9 & N/A & DON, NIV \\
\hline & Tril4 & N/A & DON, NIV \\
\hline
\end{tabular}

(Ref: Brown et al., 2004, Desjardins, 2006) 
Based on the functions described in Table 1, Foroud and Eudes predicted the trichothecene biosynthesis pathway as illustrated in Figure 3 (Foroud and Eudes, 2009). Some steps in the trichothecene biosynthesis pathway are not catalyzed by any of the known Tri genes, and genes encoding these proteins have yet to be identified. 
Figure 3: Proposed trichothecene biosynthesis pathway for DON, NIV, 4-ANIV and 15ADON. Modified from Foroud and Eudes (2009) with information added from Brown et al., 2001 and Brown et al., 2004.

The trichothecene biosynthesis pathway begins with farnesyl pyrophosphate, an intermediate substrate from the mevalonate pathway, being transformed into trichodiene by Tri5. Following a series of cyclization, hydroxylation, acetylation and esterification driven by different Tri gene products, the appropriate trichothecene is formed. 


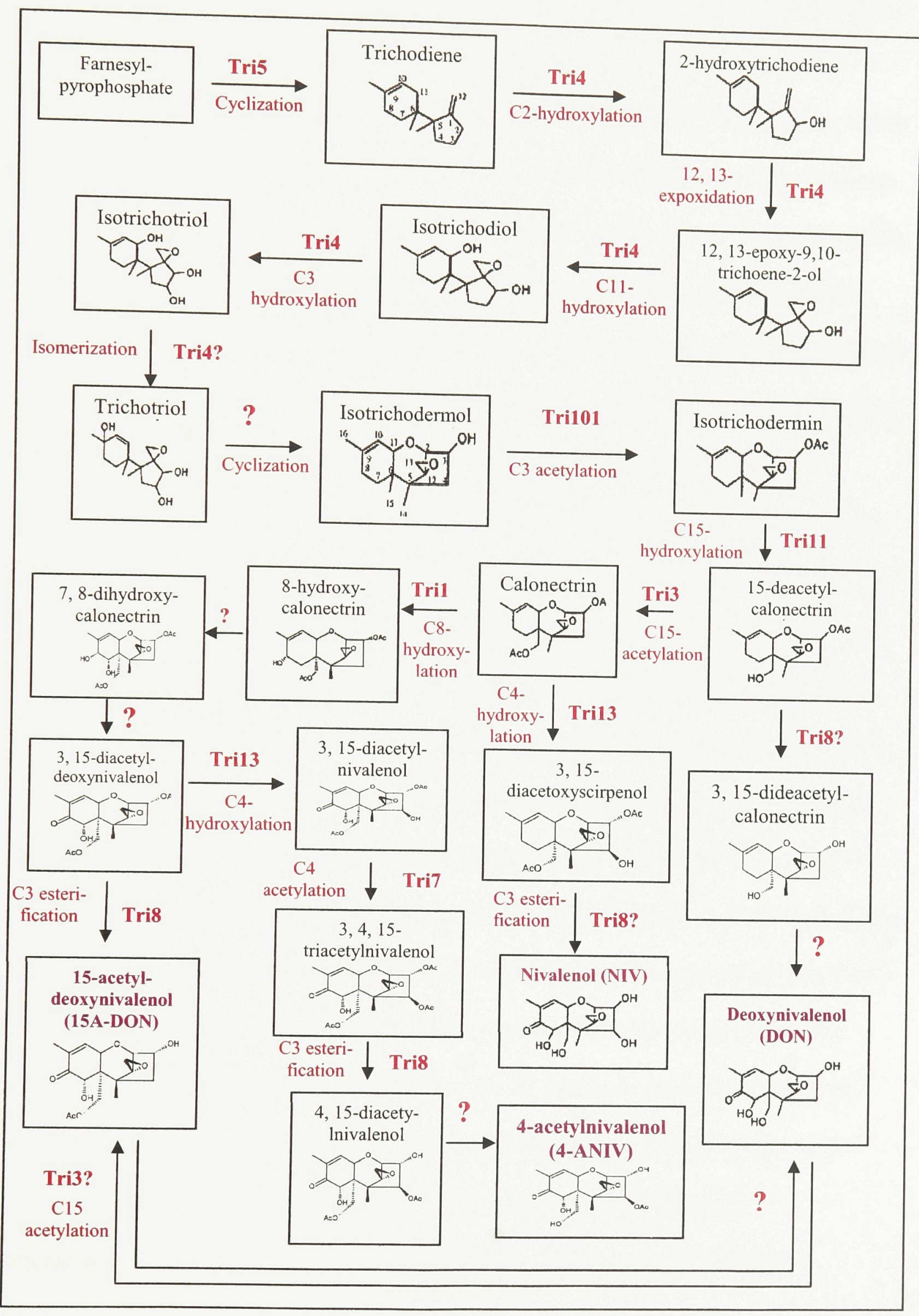


The trichothecene biosynthesis pathway in F. graminearum begins with the cyclization of the initial substrate, farnesyl pyrophosphate, into trichodiene by Tri5. Farnesyl pyrophosphate is an intermediate substrate from the acetate-mevalonate pathway, which produces isoprenoid organic compounds required for a wide range of cellular functions such as cell membrane maintenance and steroid synthesis (Suzuki and Muranaka, 2007). Following a series of hydroxylations, isomerizations, acetylations, and cyclizations, different type B trichothecenes are produced. Four of the known Tri genes are identified as cytochrome $\mathrm{P} 450$ oxygenases, a superfamily of heme-containing enzymes (Urlacher and Eiben, 2006). They generally function by introducing oxygen into substrate molecules and can catalyze a wide range of reactions, including hydroxylation of fatty acids and aromatic compounds, epoxidation of alkenes $(\mathrm{C}=\mathrm{C})$, and cleavage of an acylcarbon bond (Desjardins, 2006, Urlacher and Eiben, 2006). This explains the reoccurrence of Tri4 to drive several reactions in the early stages of the biosynthesis pathway such as hydroxylation of carbon 2, carbon 11, carbon 3 and also catalyzing the 12, 13-epoxidation reaction. Acetylation, addition of an acetyl group $\left(-\mathrm{COCH}_{3}\right)$, is the most common form of acylation observed in the pathway. While Tri3, Tri101 and Tri7 catalyze acetylation, Tri8 is the only one in this group that catalyze an esterification. The gene products of Tri101 and Tri8 both act on carbon 3, but with opposite effects: Tri101 adds an acetyl group to carbon 3 while Tri8 removes it. There have been numerous studies in the past few years to ascertain the role of regulatory genes Tril0 and Tri6, section 1.6 will summarize these studies. The other regulatory gene Tril 5 has been speculated to act as a negative regulator of the trichothecene pathway in $F$. sporotrichioides, but evidence to support this in F. graminearum has not been 
forthcoming (Alexander et al., 2004). Fg Tri12 product and its homologous counterpart in F. sporotrichioides is a trichothecene efflux pump involved in self-protection against trichothecenes (Alexander et al., 1999). The functions of Tri9 and Tril 4 have yet to be elucidated.

\subsection{Regulatory Genes of the Trichothecene Biosynthesis Pathway: Trilo and Tri6}

The trichothecene biosynthesis pathway is not constitutively active in Fusarium spp. It is activated under nutrient-limiting conditions in vitro, especially when carbon and nitrogen sources are insufficient (Pestka et. al., 1985). Possible host plant-derived inducers of DON production have been reported recently. Using a transgenic $F$. graminearum strain that harbours a Tri5 promoter-GFP construct, Gardiner et al. tested the ability of 95 different nitrogen sources to activate the DON biosynthesis pathway (Gardiner et al., 2009). It was discovered that compounds such as agmatine, arginine and guanine can induce DON production to amounts that are equal or greater than the levels of DON detected in infected wheat heads (Gardiner et al., 2009). These compounds are involved in the polyamine biosynthesis pathway of host plants, and they are capable of inducing the T-2 toxin biosynthesis pathway in F. sporotrichioides as well (Gardiner et al., 2009). Initially demonstrated in F. sporotrichioides, Tri5 is the first enzyme committed to the trichothecene biosynthesis (see Figure 3) and its expression is tightly controlled by the regulatory genes Tri10 and Tri6 (Proctor et al., 1995, Tag et al., 2001).

Initial characterization of Tri10 and Tri6 was performed in F. sporotrichioides, which sheds light on the roles of their counterparts in F. graminearum (Goswami and 
Kistler, 2004). Tril0 is located next to Tri5 in the Tri gene cluster, and encodes a protein of 420 amino acids (Tag et al., 2001). Upon deletion of this gene, Fs Tri104 did not produce T-2 toxin. Northern analysis indicated a significant decrease in the expression of all Tri genes, including the 11 that reside in the Tri gene cluster and the three outside of the Tri cluster, Tril, Tri101 and Tri15 (Tag et al., 2001, Peplow et al., 2003). In the same study, a Fs Tri6-deleted mutant also significantly decreased expression of all Tri genes with the exception of Tri10 (Peplow et al., 2003). From this, the authors suggested that while both Tri10 and Tri6 regulate the expression of Tri genes, Trilo functions upstream of Tri6 (Peplow et al., 2003). Sequence analysis of Tril0 at the amino acid level does not reveal any conserved motifs (Peplow et al., 2003).

On the other hand, sequence analysis of the 217 amino acid protein encoded by the Fs Tri6 gene revealed three imperfect repeats of $\mathrm{Cys}_{2} \mathrm{His}_{2}$ zinc finger-like motifs, each slightly differing from the general consensus of a $\mathrm{Cys}_{2} \mathrm{His}_{2}$ zinc finger motif, $\mathrm{CX}_{(2-}$ ${ }_{4)} \mathrm{CX}_{3} \mathrm{FX}_{5} \mathrm{LX}_{2} \mathrm{HX}_{(3-4)} \mathrm{H}$ (Proctor et al., 1995, Krizek et al., 1991). The cysteines (C) and histidines $(\mathrm{H})$ in the first zinc finger-like motif in Fs Tri6 are conserved but unlike the general consensus sequence where there are only three residues in between the second cysteine (C) and phenylalanine (F), there are five residues (Proctor et al., 1995). Also, the conserved leucine is replaced by another phenylalanine (Proctor et al., 1995). In the second zinc finger-like motif, phenylalanine and leucine are absent but three hydrophobic residues, methionine $(\mathrm{M})$, valine $(\mathrm{V})$ and phenylalanine, are found between the two cysteine and histidine fingers (Proctor et al., 1995). The third zinc finger-like motif is also conserved, including the phenylalanine, but leucine is replaced with a methionine (Proctor et al., 1995). Zinc finger motifs refer to a special structural domain on proteins 
which require the binding of a zinc ion for stabilization (Krishna et al., 2003). There are over 20 types of zinc-binding structural domains, for example, zinc ribbon and zinc binding loops, but the most common one is the $\mathrm{Cys}_{2} \mathrm{His}_{2}$ zinc finger motif (Krishna et al., 2003). This type of zinc finger motifs forms two $\beta$-sheets at their $N$-terminus, which arrange themselves into hairpin, finger-like structure, and also one $\alpha$-helix at their Cterminus (Krishna et al., 2003, Brayer and Segal, 2008). The side-chains of the two cysteines and two histidines are responsible for binding to the zinc ion, and in proteins with transcription factor properties, the $\alpha$-helix usually interacts with the major groove of the targeted nucleotide sequence to activate transcription (Iuchi, 2000, Krishna et al., 2003, Brayer and Segal, 2008). Previously, $\mathrm{Cys}_{2} \mathrm{His}_{2}$ zinc finger motifs have been associated with transcription factors because of their interaction with nucleotides, however, there have been increasing reports of this type of motifs interacting with RNA and also other proteins (Brown, 2005, Brayer and Segal, 2008). In the case of Fs Tri6, $\mathrm{Cys}_{2} \mathrm{His}_{2}$ zinc finger motifs mediated binding to the consensus nucleotide sequence TNAGGCCT on the promoters of Tri4 and Tri5 (Hohn et al., 1999). Using gel-shift analysis, the authors demonstrated that $F s$ Tri6 protein was able to bind to all three fragments of the Tri5 promoter that harbour the consensus sequence, as well as two consensus sequences contained in the promoter of Tri4 gene (Hohn et al., 1999). The importance of zinc finger motifs was further supported by mutational analysis. For example, Fs Tri6 protein with a C187A mutation in its third zinc finger motif failed to bind to the same promoter regions of Tri5 (Hohn et al., 1999). In order to test the binding of Fs Tri6 to DNA in vivo, the authors created numerous transgenic $F$. sporotrichioides strains in which the two consensus sequences on the Trit promoter were altered or 
deleted, and the open reading frame of Tri4 was replaced by the reporter gene $\beta$ glucuronidase (Hohn et al., 1999). The F. sporotrichioides mutant strains carrying a Tri4 promoter with the two intact consensus sequences expressed the same level of GUS as wild-type, however, in strains where one or both of the consensus sequences were missing or altered, their levels of GUS expression were at least 15-fold lower (Hohn et al., 1999). The role of Tri6 as a transcriptional regulator was further documented when it auto-activated GAL4 reporter genes when fused to a GAL4 DNA binding domain in yeast (Proctor et. al., 1995). Together with its DNA binding properties in vitro, Fs Tri6 was hypothesized to be a transcriptional regulator of some Tri genes.

Fg Tril0 and Fs Tri10 share $88 \%$ identity at the amino acid level and Fg Tri6 and Fs Tri6 proteins also share $86 \%$ identity including the three $\mathrm{Cys}_{2} \mathrm{His}_{2}$ zinc finger-like motifs (Brown et al., 2001). Despite sharing a high degree of identity, regulatory roles of Tri10 and Tri6 described in F. sporotrichioides remained to be verified in $F$.

graminearum. Recently, Seong et. al. have confirmed that, similar to F. sporotrichioides, both Fg TrilO and Fg Tri6 are required for the production of trichothecene (Seong et. al., 2009). In addition, disruption of $F g$ Tril 10 resulted in a significantly lowered expression in all of the fifteen Tri genes (Seong et. al., 2009). Likewise, disruption of Fg Tri6 significantly decreased expressions of Tri genes, with the exception of Tri10 (Seong et. al., 2009). 


\subsection{Thesis Objectives}

While it has been established that both $\mathrm{Fg}$ TrilO and Fg Tri6 are required for the activation of Tri genes, there is increasing evidence suggesting that the two genes function outside of the trichothecene biosynthesis pathway. For example, in $F$. sporotrichioides, four genes, Ibt 1 - Ibt4, which code for proteolytic enzymes such as aspartyl protease and cellulase, were down-regulated in both Fs Tri104 and Fs Tri64 strains when compared to the wild-type (Peplow et al., 2003). In the same study, expression of the gene $I b s I$, which codes for a NADH-dependent flavin oxidoreductase, was significantly reduced in Fs Tri64 strain but was not affected in Fs Tri104 strain (Peplow et al., 2003), suggesting that the two genes regulate an unique set of genes. This is further supported by a gene expression profiling study on infected wheat heads using both Tri104 and Tri64 mutant strains (Seong et al., 2009). My research was undertaken well in advance of the publication of this study, which set out to address the differential roles of the two regulatory genes, Tri10 and Tri6, of the trichothecene biosynthesis pathway in F. graminearum. To achieve this goal, the following were performed:

1) Construction of $F$. graminearum Tri10 0 and Tri6 $6 \Delta$ mutant strains.

2) Expression profile analysis in both disrupted strains under nutrient-rich and nutrientlimiting conditions were performed to identify unique and co-regulated genes.

3) Assign distinct roles for Tri10 and Tri6 in F. graminearum pathogenicity. 


\section{Chapter 2 - Materials and Methods}

\subsection{Fusarium Strains, Media and Culture Conditions}

Fusarium graminearum wild type NRRL 31084 (strain 233423) was used in this study. Fresh spores were grown in $50 \mathrm{~mL}$ of CMC liquid media (Cappellini and Peterson, $1965)$ at $170 \mathrm{RPM}, 28^{\circ} \mathrm{C}$ for 5 days. Spores were harvested by filtering through four layers of sterile cheesecloth (Fisher Healthcare, Houston, TX, USA), washed twice by centrifugation at $4000 \mathrm{RPM}$, resuspended in sterile water and stored at $4^{\circ} \mathrm{C}$ until use. $F$. graminearum wild-type, mutant and complemented strains were maintained on potato dextrose agar (PDA) with appropriate antibiotics. Mycelia of both wild-type strains were grown by inoculating 10,000 spores in $4 \mathrm{~mL}$ of first stage media (Taylor et al., 2008) for $24 \mathrm{hrs}$, washed and then transferred to grow in $4 \mathrm{~mL}$ of second stage media (Taylor et al., 2008) for 4 hrs or $12 \mathrm{hrs}$. Mycelia were washed with sterile water, harvested by vacuum filtration using MF-Millipore membrane filters (Bedford, MA, USA), and stored at $80^{\circ} \mathrm{C}$ until use.

\subsection{Gene Disruption Constructs, Protoplast Preparation and Fungal Transformation}

Tri10 and Tri6 disruption constructs consisting of the selection marker, hygromycin, flanked by $1000 \mathrm{bp}$ of each 5'- and 3'- region of the respective genes were created using fusion PCR as described in Figure 4. Primers used to make these constructs are listed in Table A1 and Table A2 of the Appendix. PCR master mix consisted of 0.2 $\mu \mathrm{M}$ forward primer, $0.2 \mu \mathrm{M}$ reverse primer, $0.2 \mathrm{mM}$ dNTPs, $1.5 \mathrm{mM} \mathrm{MgCl}_{2}, 50 \mathrm{ng}$ of DNA template and 1.8 units of Expand long template PCR system (Hoffmann-La Roche Limited, Mississauga, ON, Canada). Protoplasts were made by digesting $1-5 \times 10^{8}$ germinated spores using an enzyme solution consisting of $5 \%$ Driselase from 
Basidiomycetes sp. (Sigma-Aldrich Corporation, St. Louis, MO, USA), $1.0 \mathrm{mg}$ of

Chitinase from Streptomyces sp. (Sigma-Aldrich Corporation, St. Louis, MO, USA), and 100 mg Lysing enzymes from Aspergillus sp. (Sigma-Aldrich Corporation, St. Louis, $\mathrm{MO}, \mathrm{USA}$ ) in $20 \mathrm{~mL}$ of $1.0 \mathrm{M}$ of $\mathrm{NH}_{4} \mathrm{Cl}_{2} \mathrm{pH} 5.0$ (Wiebe et al., 1997) at $100 \mathrm{RPM}$ at $30^{\circ} \mathrm{C}$ for $2.5 \mathrm{hrs}$. After washing with 1.0M N $\mathrm{NH}_{4} \mathrm{Cl}_{2} \mathrm{pH} 5.0$, the protoplasts were resuspended in $300 \mu \mathrm{L}$ of STC buffer (1.2M sorbitol, $10 \mathrm{mM}$ Tris $\mathrm{pH} 8.0,50 \mathrm{mM} \mathrm{CaCl}_{2}$ dihydrate) and diluted to $1 \times 10^{8}$ protoplasts per mL with storage buffer $(800 \mu \mathrm{L} \mathrm{STC}, 200 \mu \mathrm{L}$ of $30 \%$ $\mathrm{PEG}, 10 \mu \mathrm{L}$ of DMSO). Protoplasts were aliquoted into $100 \mu \mathrm{L}$ aliquots and stored in $80^{\circ} \mathrm{C}$ until use.

Fungal transformation was carried out by incubating $100 \mu \mathrm{L}$ protoplasts with 500 ng DNA of the disruption construct for 30 mins at room temperature containing STC and $30 \%$ PEG. After incubation, $2 \mathrm{~mL}$ of $30 \%$ PEG and $4 \mathrm{~mL}$ of STC were added and subsequently the entire transformation mixture was added to $200 \mathrm{~mL}$ of Regeneration media $(0.6 \mathrm{~g}$ of yeast extract, $0.6 \mathrm{~g}$ of casein hydrolysate, $9.2 \mathrm{~g}$ of agar, $164.3 \mathrm{~g}$ of sucrose in $600 \mathrm{ml}$ of water, autoclaved), mixed and poured into petri dishes. The plates were incubated at $28^{\circ} \mathrm{C}$ for 24 hours before adding a $10 \mathrm{~mL}$ overlay consisting of $1 \%$ agar and $100 \mu \mathrm{g} / \mathrm{mL}$ of Hygromycin B from Streptomyces sp. (Calbiochem, Gibbstown, NJ, USA) or $100 \mu \mathrm{g} / \mathrm{mL}$ of G418 geneticin (Sigma-Aldrich Corporation, St. Louis, MO, USA).

\subsection{Complementation of Tri10 and Tri6 Deleted Mutant Strains}

To construct the "addback" vector for a disrupted gene mutant, the gene of interest and its native promoter were amplified from $F$. graminearum genomic DNA, digested with EcoRI and EcoRV restriction enzymes (New England Biolabs Inc., 
Ipswich, MA, USA), and ligated to the EcoRI-, EcoRV-digested pII99 vector (Namiki et al., 2001). The $5.3 \mathrm{~Kb}$ pII99 plasmid is a derivative of pSP72 (Promega Corporation, Madison, WI, USA) that expresses the geneticin gene under the control of the trpC promoter (Namiki et al., 2001). The pII99 vector map is available in Figure A1 in the Appendix. Protoplasts were made from Tri10 04 and Tri6 64 mutant spores, and $1 \mu \mathrm{g}$ DNA of each addback construct was transformed into protoplasts. The primers used for amplifying Tri6 promoter-ORF as well as for Tri10 promoter-ORF are listed in Table A3 in the Appendix.

\subsection{DNA Isolation and Analysis}

Frozen mycelia samples were ground to a powder using liquid nitrogen and DNA was extracted by using DNeasy Plant Mini Kit (Qiagen, Valencia, CA, USA). Deleted mutants were confirmed by PCR using hygromycin primers (Hyg P5, Hyg P6), Tri10 ORF primers (Tri10 orf F and Tri10 orf R), and Tri6 ORF primers (T6 Fsp and T6 End R). In addition to the mentioned primers, complemented mutants were also screened for the presence of geneticin, which is the selection marker of the addback vector pII99, using geneticin primers (Geneticin ENTR and Geneticin Down). PCR parameters consisted of a 2-minute $94^{\circ} \mathrm{C}$ denaturing step, followed by 31 cycles of 30 secs at $94^{\circ} \mathrm{C}$, 30 secs at $54^{\circ} \mathrm{C}$, and 2 mins at $68^{\circ} \mathrm{C}$. The ingredients of the PCR master mix were identical to that discussed in Section 2.2., but 0.6 units of Taq polymerase was used instead of Expand long template PCR system. Primer sequences are available in Table A4 in the Appendix. GeneRuler ${ }^{\mathrm{TM}} 1 \mathrm{~Kb}$ Plus DNA ladder (Fermentas Canada Inc., Burlington, ON, Canada) was used for size confirmation of PCR products. 
DNA blot analysis was performed on both Tri104 or Tri64 mutant strains.

Genomic DNA of wild-type and Tri104 mutant strain were digested with SacI enzyme (New England Biolabs Inc., Ipswich, MA, USA), while wild-type and Tri64 genomic DNA were digested with NotI enzyme (New England Biolabs Inc., Ipswich, MA, USA). A total of $4 \mu \mathrm{g}$ of each digested DNA sample was loaded onto the gel and separated at 80 volts for 4 hours. Subsequently, the gel was treated with $0.2 \mathrm{M} \mathrm{HCl}$ for 15 mins followed by incubation with $1.5 \mathrm{M} \mathrm{NaCl}, 0.5 \mathrm{M} \mathrm{NaOH}$ for 45 mins with gentle shaking to ensure the denaturation of double stranded DNA. The gel was neutralized with $1 \mathrm{M}$ Tris $\mathrm{pH}$ 7.4, $1.5 \mathrm{M} \mathrm{NaCl}$ for 30 mins, and DNA was transferred onto a nitrocellulose membrane (Millipore, Bedford, MA, USA) overnight (Maniatis et al.,1989). Following the transfer, the nitrocellulose filter was baked in an $80^{\circ} \mathrm{C}$ oven for $1 \mathrm{hr}$ and soaked in 20X SSC (3M sodium chloride, $300 \mathrm{mM}$ sodium citrate) for hydration prior to storing between Whatman 3MM filter papers (GE Healthcare, Buckinghamshire, UK). For hybridization of hygromycin radioactive probe to membrane, see section 2.6. (Northern analysis and hybridization of radiolabeled probes).

\subsection{RNA Extraction and Microarray Analysis}

RNA was extracted using the RNeasy Plant Mini Kit (Qiagen, Valencia, CA, USA). Integrity of RNA was measured by Agilent 2100 Bioanalyzer (Agilent Technologies Inc., Santa Clara, CA, USA). RNA samples were then transcribed into cDNA using a reverse transcriptase provided in the Quick Amp Labeling Kit (Agilent Technologies Inc., Santa Clara, CA, USA) and subsequently converted into cRNA using T7 RNA polymerase and cyanine 3-labeled CTP or cyanine 5-labeled CTP provided by 
the Agilent two-color RNA spike-in kit (Santa Clara, CA, USA). Purification of cRNA was carried out using RNeasy mini spin columns from Qiagen (Valencia, CA, USA). Hybridization of purified cRNA to $F$. graminearum Agilent microarray slide was performed using the Gene Expression Hybridization Kit (Agilent Technologies Inc., Santa Clara, CA, USA). Each array consists of approximately 14,666 oligos including 1,417 spike-in and negative controls, and the remaining oligos represent a number of 13,918 expressed sequence tags with three 60mer-oligos representing each gene. Finally, the array slide was washed with Wash Buffer provided by the manufacturer (Agilent Technologies Inc., Santa Clara, CA, USA).

Hybridization signals were scanned using a GenePix Professional 4200A Scanner (Molecular Devices, Sunnyvale, CA, USA) and signal intensities were quantified using the software GenePix Pro 6 (Molecular Devices, Sunnyvale, CA, USA). Microarray data were imported into Acuity 4.0 (Molecular Devices, Sunnyvale, CA, USA) for assessment and analysis. Two-dye microarray data are subjected to non-linear dye bias, where dye intensity does not proportionately correlate to the level of gene expression, therefore rendering unreliable data (Oshlack et al., 2007). Lowess Normalization was applied to raw microarray data to eliminate variations generated from dye bias, so that the only variation between gene expressions would be biological-based. Three biological replicates and two technical replicates for each experiment were compared, and only substances with a p-value $<0.001$ were selected. Prior to combining technical and biological replicates, hierarchical clustering was performed on Acuity software to verify that all the same arrays were aligned together. Combined data, expressed as $\log _{2}$, were 
then transferred onto Microsoft Excel to determine genes that were commonly or differentially regulated in Tri104 and Tri64.

Genes with an expression of $\log _{2} \geq 1$ (two-fold or more) in wild type, Tri104 or Tri64 mutants were considered up-regulated genes in each strain. To identify genes that were specifically up-regulated in WT, genes with an expression of $\log _{2} \geq 1$ in wild type but $\log _{2} \leq 1$ in Tri104 and Tri64 mutants were selected. Similar analysis was performed to identify genes that were specifically up-regulated in Tri104 or Tri64. To identify genes that were up-regulated in both WT and Tri104 only, genes with an expression of $\log _{2} \geq 1$ in WT and Tri104 but $\log _{2} \leq 1$ in Tri64 were selected. Similar analysis was performed to identify genes that were up-regulated in WT and Tri64 only, as well as genes that were up-regulated in the two mutants only. Genes with an expression of $\log _{2} \geq$ 1 in WT, Tri10 and Tri64 were regarded as up-regulated genes in all three strains.

Genes with an expression of $\log _{2} \leq-1$ in wild type, Tri104 or Tri64 were considered down-regulated genes in each strain. To identify genes that were specifically down-regulated in WT, genes with an expression of $\log _{2} \leq-1$ in WT but $\log _{2} \geq-1$ in Tri104 and Tri64 were selected. Similar analysis was performed to identify genes that were specifically down-regulated in Tri104 or Tri64. To identify genes that were downregulated in both WT and Tri10 only, genes with an expression of $\log _{2} \leq-1$ in WT and Tri10 but $\log _{2} \geq-1$ in Tri6ム were selected. Similar analysis was performed to identify genes that were down-regulated in WT and Tri64 only, as well as genes that were down- 
regulated in the two mutants only. Genes with an expression of $\log _{2} \leq-1$ in WT, Tril04 and Tri64 were regarded as down-regulated genes in all three strains.

\subsection{Northern Analysis and Hybridization of Radiolabeled Probes}

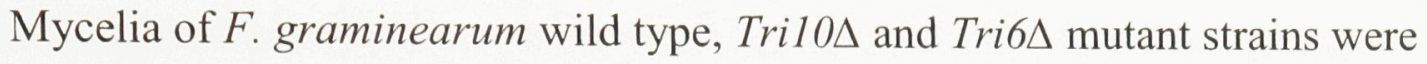
grown in the two-stage media as described in Section 2.1. for microarray and Northern analyses. RNA samples subjected to microarray analysis were extracted from mycelia incubated in $2^{\text {nd }}$ stage media for 4 hours whereas RNA samples subjected to Northern analysis were extracted from mycelia incubated in $2^{\text {nd }}$ stage media for 4 hours and 12 hours. RNA extraction was performed according to section 2.5. Northern analysis was performed on selected genes to confirm expression levels indicated in the microarray data. A total of $10 \mu \mathrm{g}$ RNA of each sample was loaded and separated on a $1 \%$ formaldehyde gel at 65 volts for 2-3 hours (Maniatis et al., 1989). Following electrophoresis, the gel was rinsed in deionized water and incubated in 10X SSC for 45 mins with gentle shaking. RNA was transferred onto an Immobilon-NY+ charged nylon membrane (Millipore, Bedford, MA, USA) by overnight capillary transfer (Maniatis et al., 1989). RNA was immobilized on the nylon membrane by exposure to ultraviolet light in an ultraviolet crosslinker (model CL-1000, Ultra-Violet Products Limited, Science Park, Cambridge, UK). The membrane was stored between two Whatman 3MM filter papers at room temperature until hybridization.

Prior to hybridization of radiolabeled probes, the nitrocellulose filter or nylon membrane was incubated with $10 \mathrm{~mL}$ of ExpressHyb Hybridization Solution (Clontech, 
Mountain View, CA, USA) at $65^{\circ} \mathrm{C}$ for $1 \mathrm{hr}$ to block non-specific hybridization.

Meanwhile, the radioactive probe was prepared in a random priming reaction with $25 \mathrm{ng}$ of DNA template and $\left[\alpha^{32} \mathrm{P}\right]$-CTP (Perkin Elmer, Boston, MA, USA) using the reagents provided by the NEBlot Kit (New England Biolabs Inc., Ipswich, MA, USA) at $37^{\circ} \mathrm{C}$ for $1 \mathrm{hr}$. The hygromycin probe was used in the Southern analysis whereas the probes Tri10, Tri6, Tril and Fg00007 were used in Northern analysis. Primers used to amplify nonradiolabeled probe templates are listed in Table A5 in the Appendix. The probes were purified using Illustra NICK columns (GE healthcare, Buckinghamshire, UK). Specific activity of the probe was measured by Cerenkov counting by spotting $1 \mu \mathrm{L}$ of the probe to a $1.5 \mathrm{~mm} \times 1.5 \mathrm{~mm}$ Whatman $3 \mathrm{MM}$ filter paper and subjected to a liquid scintillation analyzer (model 1900 TR, Packard BioScience Company, Meriden, Connecticut, USA). A total of $2.0 \times 10^{7} \mathrm{CPM}$ of probe was mixed with $200 \mu \mathrm{L}$ salmon sperm DNA, boiled and cooled on ice for 5 mins and then added to the hybridization solution incubated with the membrane at $65^{\circ} \mathrm{C}$ overnight. After hybridization, membranes were washed with $2 \mathrm{X}$ SSC, $0.1 \%$ SDS at room temperature for 30 mins and re-washed with the same buffer for $1 \mathrm{hr}$ at $65^{\circ} \mathrm{C}$. Membranes were wrapped in plastic wrap and exposed to Kodak BioMax MS film (Carestream Health Inc., Rochester, NY, USA) with a BioMax MS intensifying screen (Eastman Kodak Company, Rochester, NY, USA), and stored in $-80^{\circ} \mathrm{C}$ overnight. The film was developed using a medical film processor (model SRX-101A, Konica Minolta Medical and Graphic Inc., Taiwan) and depending on the band intensity, another film was exposed to the membrane for an alternate amount of time. 


\subsection{Wheat Infection Assays}

Wheat seeds, variety Roblin, were germinated on $1 \%$ water agar and transplanted into soil that had been fertilized with $20 \%$ nitrogen, $20 \%$ phosphorous, and $20 \%$ potassium. They were grown in a chamber that provided 16 hours of full spectrum light at a constant temperature of $22^{\circ} \mathrm{C}$ followed by 8 hours of darkness at a temperature of $15^{\circ} \mathrm{C}$ for six weeks. During anthesis, different strains of $F$. graminearum were inoculated into the floret at a concentration of 1000 spores per $10 \mu \mathrm{L}$, as described in Hou et al. (2002). Inoculated wheat heads were marked and labelled, then placed in a growth room with 16 hours of full spectrum light at a day temperature of $20^{\circ} \mathrm{C}$, followed by 8 hours of darkness at a night temperature of $15^{\circ} \mathrm{C}$ for 21 days. For the first 48 hours, the infected wheat plants were exposed to water mist every 15 minutes at 30 second intervals, but for the remaining 19 days, the plants were moved to a dry area where disease symptoms were observed. Pictures of infected wheat heads 21 days post inoculation were taken using a Canon PowerShot S50 camera (Canon Canada Inc., Mississauga, ON, Canada).

\subsection{Secondary Metabolite Analysis}

Mycelia of F. graminearum wild type, Tri10 10 and Tri6 $\Delta$ mutant strains were grown in first stage media for 24 hours at $28^{\circ} \mathrm{C}, 170 \mathrm{RPM}$ prior to incubation in second stage media under the same conditions for 12 hours. $1 \mathrm{~mL}$ of filtrate was filtered through $0.2 \mu \mathrm{m}$ Nylon Syringe filters (Mandel Scientific Company Inc., Guelph, ON, Canada). The sample for analysis was prepared by mixing $450 \mu \mathrm{L}$ of the filtrate with $150 \mu \mathrm{L}$ of methanol and subjected to HPLC analysis using a 5um Hypersil ODS column (Thermo Fisher Scientific Inc., Waltham, MA, USA) connected to an AKTA P-900 purifier (GE 
Healthcare, Buckinghamshire, UK). Trichothecenes were separated by employing a water: methanol gradient of 85: 15 to $60: 40$ over 25 minutes at a flow rate of $1 \mathrm{~mL} / \mathrm{min}$. It is detected by Monitor UV-900 at 220nm (GE Healthcare, Buckinghamshire, UK). The retention time for $15-\mathrm{ADON}$ was 11.3 mins under these conditions as calibrated by 15 ADON standard (provided by Barbara Blackwell, AAFC, Ottawa). 


\section{Chapter 3 - Results}

\subsection{Fg Tri10 is Involved in Pathogenicity}

\subsubsection{Construction of F. graminearum Tri104 mutant strain}

To investigate the effects of Tril0 in F. graminearum, the Tril0 coding region was replaced with hygromycin using homologous recombination. The construct used to replace Tri10 was made as described in Figure 4. Three polymerase chain reactions (PCRs) were performed to amplify three chimeric gene fusions with overlapping sites of each other. Specifically, the first PCR product consisted of $1 \mathrm{~kb}$ of 5 ' flanking region of Tril0 with 18 bp of hygromycin at its 3' end, the second PCR fragment consisted of $1 \mathrm{~kb}$ of 3' flanking region of Tril0 with 18 bp of hygromycin at its 5' end. The third PCR fragment was the hygromycin gene with overhangs homologous to the 3 ' end of the 5 ' flanking region and the 5' end of the 3' flanking region of Tri10. The deletion construct was achieved with the combination of all three PCR products and amplification with flanking primers. 
Figure 4: Generation of Tri10 disruption construct.

Black solid line represents 5' element of Tri10, red solid line represents the hygromycin gene and blue solid line represents 3' element of Tri10. Using primers P1 and P2, the 5' element of Tri10 consisting of $1000 \mathrm{bp}$ upstream of the Tril0 coding region and $18 \mathrm{bp}$ of the 5' end of hygromycin gene was amplified. Using primers P5 and P6, the hygromycin gene consisting of overlapping regions with the 5' and 3' elements of Tril0 was amplified. Using primers P3 and P4, the 3' element of Tri10 consisting of $1000 \mathrm{bp}$ downstream of the Tri10 coding region and $18 \mathrm{bp}$ of the 3' end of the hygromycin gene was amplified. The final disruption construct consisting of 5' element of Tri10 hygromycin - 3' element of Tri10 was amplified using the P1 and P4 primers. Sequences of the primers can be found in Table A1 in the Appendix. 


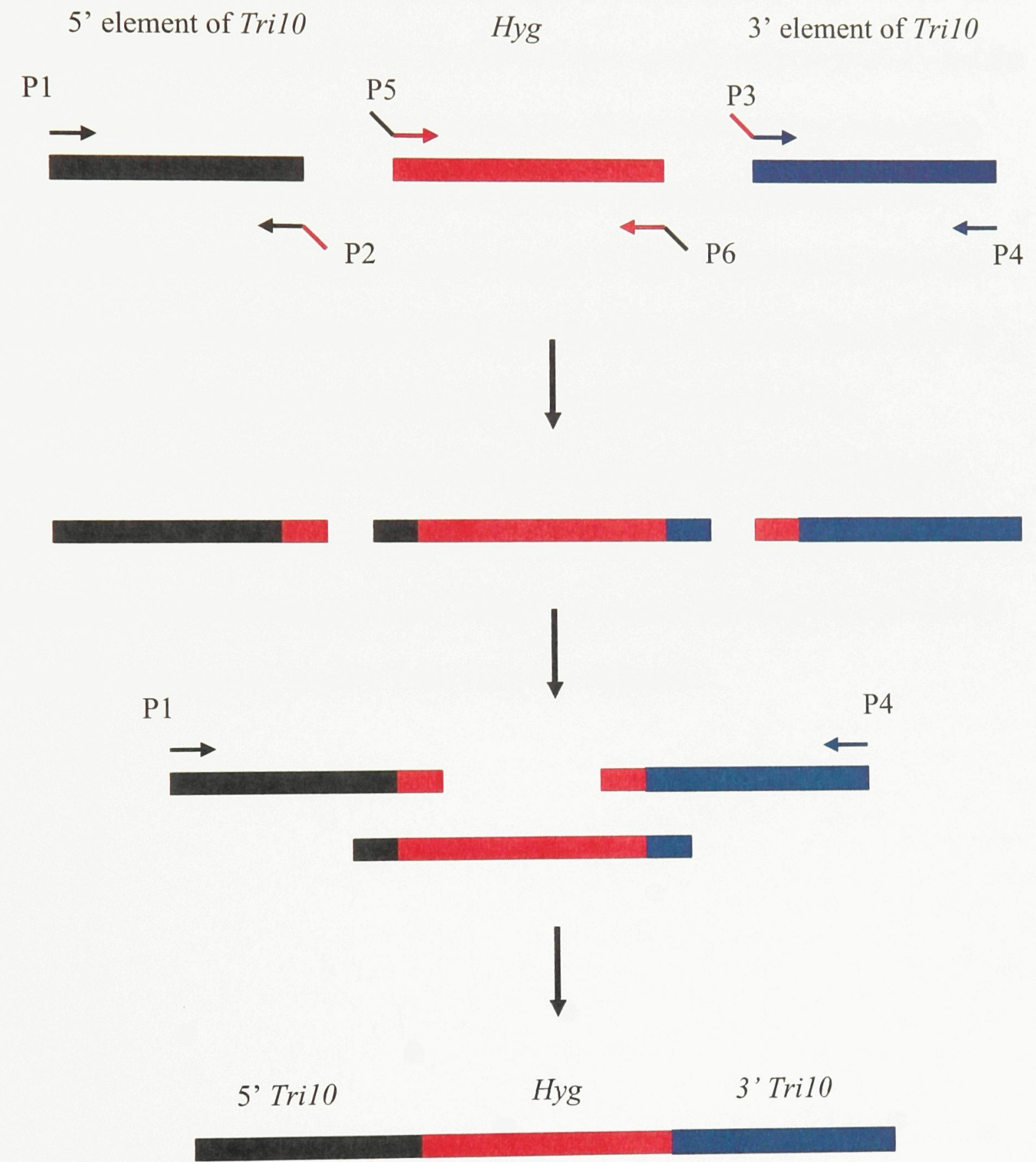


Hygromycin-resistant transformants were screened for the absence of the Tri10 gene, as well as the presence of the hygromycin selection marker, and at least one of them verified to have the targeted gene disrupted. Figure 5 represents the PCR confirmation of Tril04 mutant strain for the absence of Tril 10 using Tril0 gene-specific primers (lane 2), and the presence of hygromycin (lane 6). Southern analysis of the Tri104 mutant strain using hygromycin as probe indicated that only one copy of the disruption construct was incorporated into the genome (lanes 1 and 2, Figure 6). The Tri104 mutant strain was then complemented with plasmid ( $p$ Tri10) consisting of the Tri10 gene and $1 \mathrm{~Kb}$ of its promoter, and a second selection marker, Geneticin, into the genome. This complemented strain, denoted as Tri104-AB, harboured hygromycin from the gene replacement (lane 7, Figure 5), Tri10 gene (lane 3, Figure 5) and geneticin (lane 11, Figure 5) from $p$ Tri10 addback plasmid. The primers used in the verification of Tril0د and Tri10 $\triangle-A B$ strains are listed in Table A4 of the Appendix. 
Figure 5: Confirmation of Tril0 disruption and complementation in F. graminearum. DNA was extracted from wild type, Tri10 10 and Tri10 $-A B$ strains and subsequently used as templates for PCR confirmation of Tril0 disruption and complementation.

Lanes 1-4: WT, Tri104, Tri104-AB transformant \#9, and water control, respectively, screened with Tril0 gene specific primers, Tril0 orf $F$ and Tril0 orf $R$. The PCR product at $\sim 1200 \mathrm{bp}$ in lanes 1 and 3 represents Tri10 gene, with an expected size of $1263 \mathrm{bp}$. Lanes 5-8: WT, Tri10 , Tri10 $-A B$ transformant \#9, and water control, respectively, screened with hygromycin primers, Hyg P5 and Hyg P6. The PCR product at $\sim 1800$ bp in lanes 6 and 7 represents hygromycin, with an expected size of 1822 bp.

Lanes 9-12: WT, Tri10 4 , Tri10 $-A B$ transformant \#9, and water control, respectively, screened with geneticin primers, Geneticin ENTR and Geneticin Down. The PCR product at $\sim 1200 \mathrm{bp}$ in lane 11 represents geneticin, with an expected size of $1283 \mathrm{bp}$. 


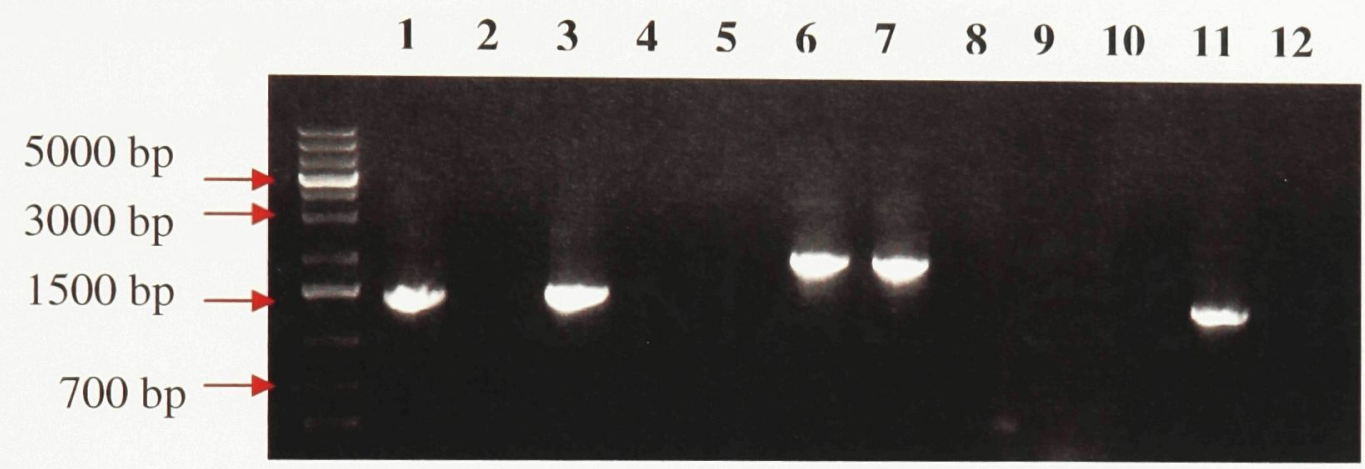


Figure 6: DNA blot analysis of Tri10 10 and Tri6 $\Delta$ mutant strains.

To determine the number of disruption construct integrated into the genome, $4 \mu \mathrm{g}$ of SacI-digested wild type and Tri10 $\mathrm{DNA}$ along with $4 \mu \mathrm{g}$ of Not $\mathrm{I}$-digested wild type and Tri64 DNA were hybridized with a hygromycin probe.

The single band at 10,000 bp present in Tril04 digested with SacI indicates that only one disruption construct was incorporated into the Tril04 genome.

The single band at $\sim 8,000$ bp present in Tri64 digested with NotI indicates that only one disruption construct was incorporated into the Tri64 genome.

Loading control for the hygromycin probe was monitored by $1 \mathrm{ng}, 2 \mathrm{ng}$, and $10 \mathrm{ng}$ of the PCR product hygromycin. 


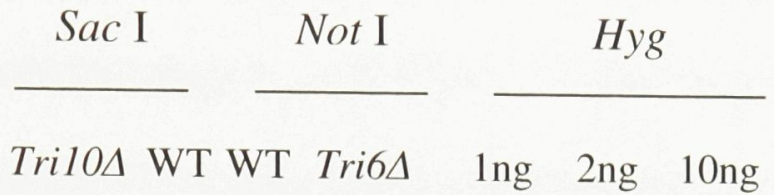
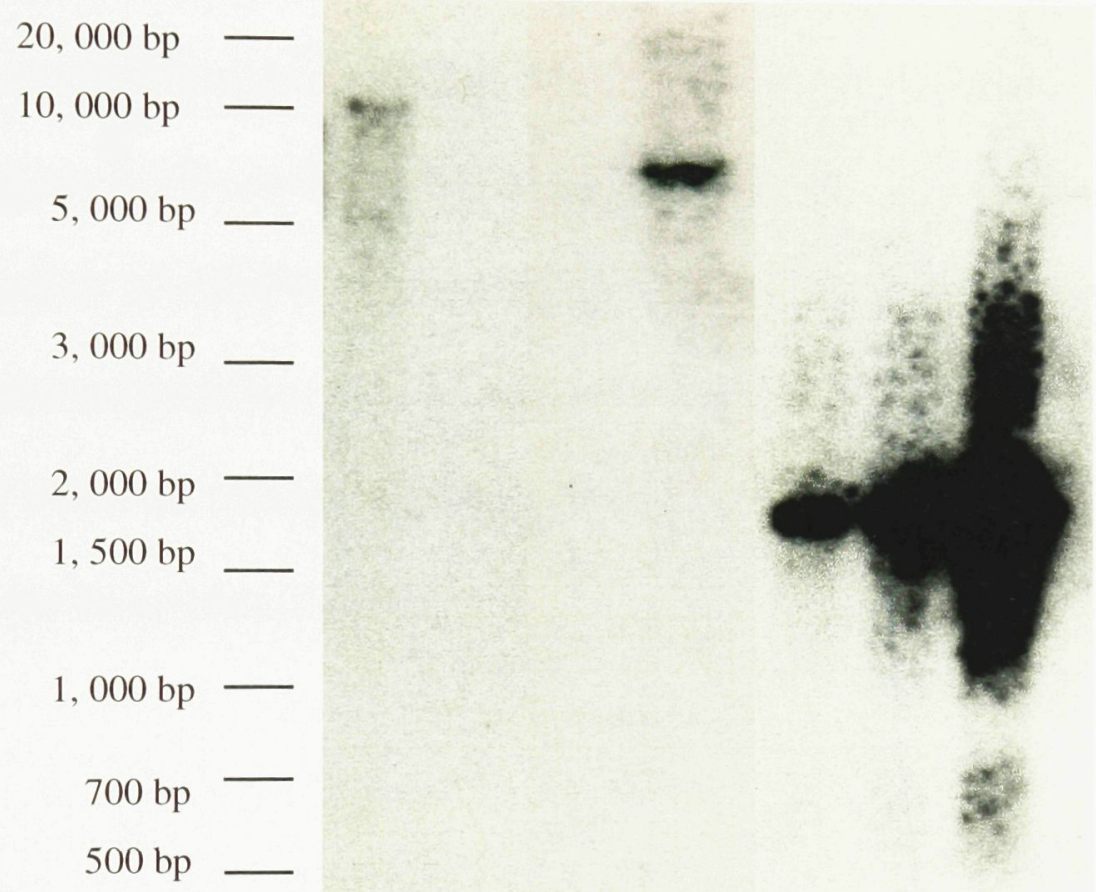


\subsubsection{Fg Tri104 does not produce trichothecene derivative 15-ADON}

A distinctive phenotype of $F$. graminearum strain 233423 is its ability to produce 15ADON when cultured under nutrient-limiting conditions in vitro. To determine the involvement of Tril0 in the production of trichothecene in F. graminearum, the filtrates of Fg Tril04 and Fg Tri104- $A B$ strains cultured under DON-inducing conditions were subjected to secondary metabolite analysis using HPLC. Figure $7 \mathrm{~b}$ shows that 15 -ADON standard elutes at approximately 11.11 mins when added to wild type $F$. graminaerum grown in non DON-inducing media (15-ADON standard provided by Barbara Blackwell, AAFC). Figure 8, 15-ADON was synthesized in the WT strain (panel A), but Fg Tri104 did not produce $15-\mathrm{ADON}$, as indicated by the absence of a peak at the retention time indicated above (panel B). The synthesis of $15-\mathrm{ADON}$ from the Tril0 complemented strain $F g$ Tri10 $-A B$ (panel C) indicated that Trilo contributes to the activation of the trichothecene biosynthesis pathway. 
Figure 7: HPLC chromatograms of 15-ADON standard.

In order to identify the elution time of 15-ADON standard, HPLC chromatogram of wild type $F$. graminearum grown in non DON-inducing media was compared to that of wild type $F$. graminearum grown under the same conditions spiked with 15-ADON (provided by Barbara Blackwell, AAFC). The $\mathrm{x}$-axis indicates retention time whereas the $\mathrm{y}$-axis represents absorbance of light transmitted in mAU.

A) Chromatogram of WT F. graminearum in non DON-inducing media for 35 hours. A solvent peak was observed at around 2 mins.

B) Chromatogram of WT F. graminearum in non DON-inducing media for 35 hours spiked with $150 \mu \mathrm{g}$ of $15-\mathrm{ADON}$. In addition to the solvent peak observed at 2 mins, an additional peak was also observed at 11.1 mins, as indicated by the red arrow. This additional peak represents $15-\mathrm{ADON}$. 


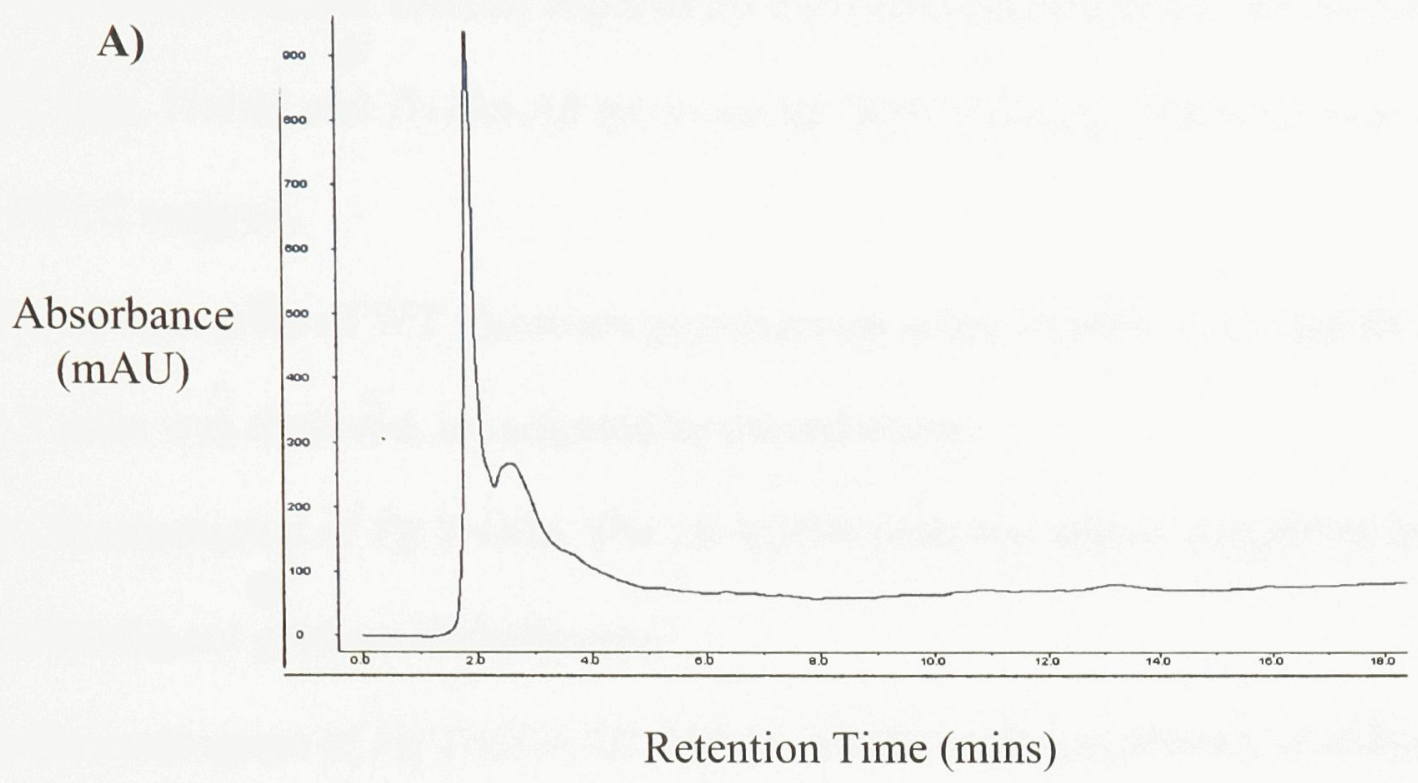

B)

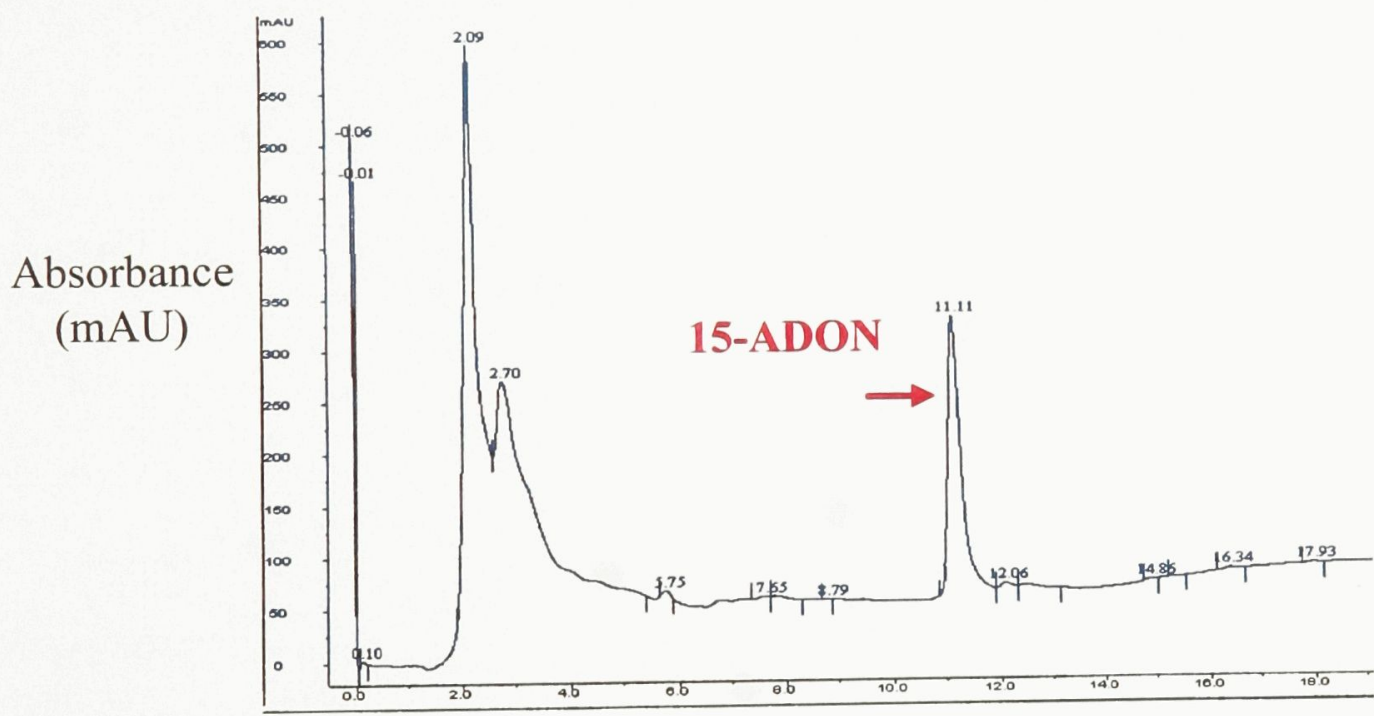

Retention Time (mins) 
Figure 8: HPLC chromatograms of $F$. graminearum Tri10 4 and Tri10 $-A B$ strains cultured under DON-inducing conditions.

To determine whether Tril0 is required for trichothecene production, the media filtrate of wild type, Tri10 4 and Tri10 $\triangle-A B$ grown under DON-inducing conditions were subjected to HPLC analysis.

A) Chromatogram of WT Fusarium graminearum strain 233423. A 15-ADON peak at 11.3 mins was observed, as indicated by the red arrow.

B) Chromatogram of $\mathrm{Fg}$ Tri104. The 15-ADON peak was absent, suggesting that $\mathrm{Fg}$ Tril04 did not produce trichothecene.

C) Chromatogram of Fg Tri104-AB. The 15-ADON peak was present, as indicated by the red arrow, suggesting that the Tri10 complemented strain was able to produce trichothecene. 

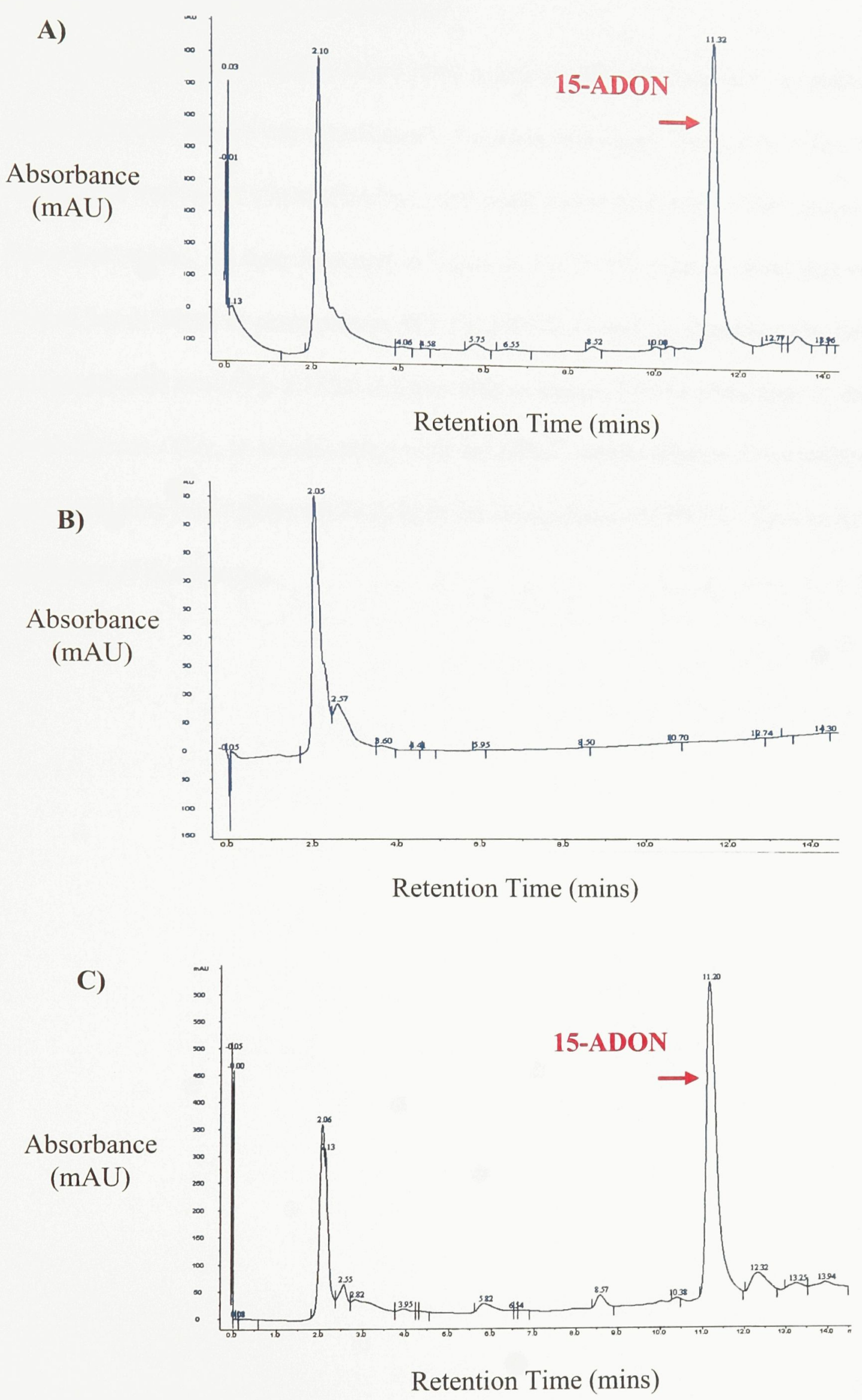


\subsubsection{Fg Tri10 contributes to virulence}

To determine whether Tril0 plays a role in pathogenicity of $F$. graminearum, wheat infection assays were performed. For each pathology assay, five wheat heads of a susceptible variety of wheat (Roblin) were point inoculated with $\sim 1000$ spores of Fusarium strains. As demonstrated in Figure 9, Fg Tri104 mutant strain was non-virulent (wheat heads 3 and 4) compared to WT Fg 233123 (1 and 2). Significantly, the complemented strain Fg Tri10 $\triangle-A B$ was able to rescue Tri10 4 phenotype (5 and 6) and cause disease. This, in combination with the HPLC results (Figure 8) conclusively suggested that Tri10 plays a role in both the biosynthesis of DON, which in turns affects virulence of this fungus. 
Figure 9: Fg Tril0 contributes to virulence in F.graminearum

Representative wheat heads (variety Roblin) after infection with WT, Tri104 and Tri10 $\triangle-A B$ strains.

1, 2: Wheat heads infected with $F$. graminearum WT strain. Twenty-one days subsequent to the initial inoculation, both infected wheat heads were completely bleached.

3, 4: Wheat heads infected with Fg Tri104 strain. The Tril04 strain was able to infect wheat heads but could not spread beyond the site of infection, as indicated by the bleaching of the inoculated spikelets only.

5, 6: Wheat heads infected with Fg Tril0 $-A B$ transformant \#9. The Tril0 complemented strain was able to infect and rescue mutant phenotype to spread beyond the site of infection, as indicated by the bleaching of the entire wheat head.

Picture was taken 21 days post infection. This experiment was repeated three times with similar results. Red arrow indicates site of inoculation. 


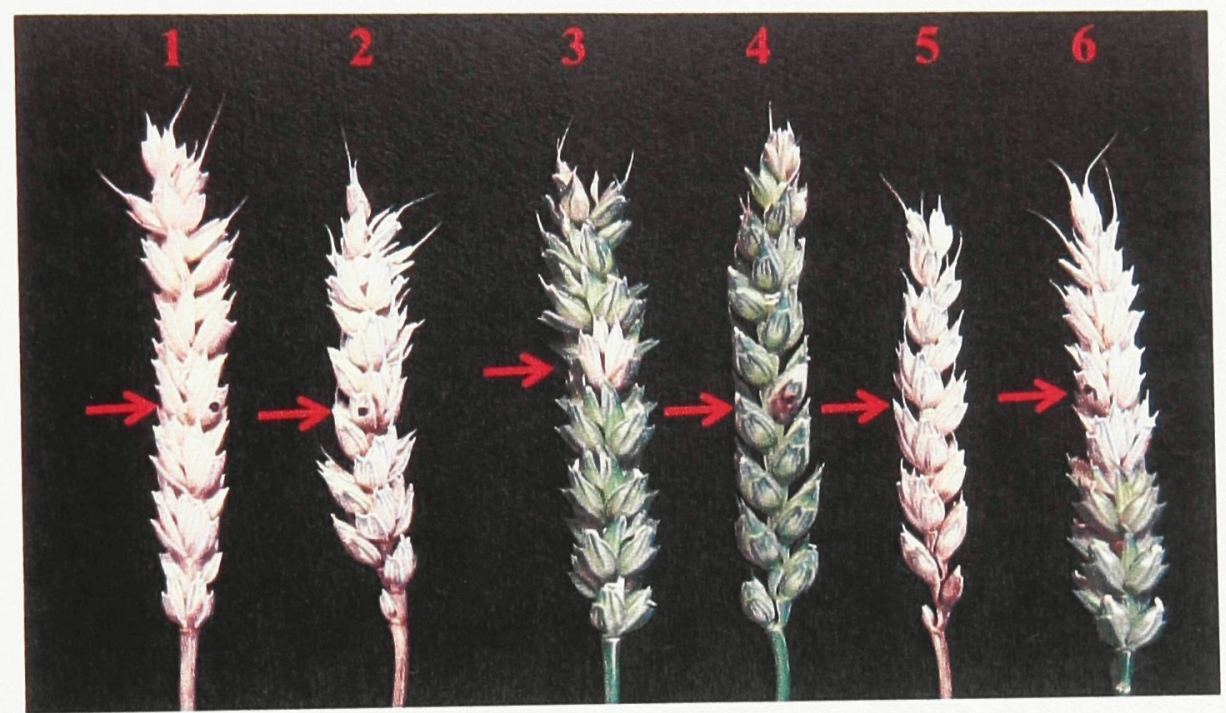




\subsection{Fg Tri6 is Involved in Pathogenicity}

\subsubsection{Disruption of Fg Tri6 using Homologous Recombination}

Fg Tri64 strain was constructed by homologous recombination using a similar fusion PCR method used to generate the Tril0 deletion mutant. However, the structure of the construct was slightly different as shown in Figure 10. Unlike Tri10 1 where the entire Tril0 open reading frame was replaced by hygromycin, recombination occurred at 107 bp inside of the ATG start site of Tri6 and 65 bp from the TGA stop codon; consequently, only 485 bp of the 657 bp Tri6 was replaced by hygromycin, as shown in Figure 10a. Figure $10 \mathrm{~b}$ presents a PCR confirmation of Fg Tri64 mutant strain, where a 1889 bp product was amplified using Tri6-specific primers (lane 2). Since Tri6-specific primers, Tri6 Fsp and Tri6 End R, anneal to regions outside the recombination sites (see Figure 10a), the entire 1822bp hygromycin gene and the $67 \mathrm{bp}$ from Tri6 were amplified as a PCR product with the size of $1889 \mathrm{bp}$. Using hygromycin-specific primers, the 1822bp hygromycin was also amplified (lane 6, Figure 10b). Similar to Tri104, Southern analysis revealed that a single copy of the disruption construct was integrated into the genome, indicating that Tri6 was the only gene disrupted during the fungal transformation and recombination process (Figure 6). Complementation of Fg Tri64 mutant strain was accomplished by re-introducing a $p$ Tri $6 H A$ addback plasmid into the Tri6 deleted strain. The add back construct with Geneticin as a selection marker, consisted of $1 \mathrm{~Kb}$ of the Tri6 promoter, and the Tri6 gene fused to a hemagglutinin (HA) epitope tag at the C-terminus. This complemented Fg Tri64-AB strain was screened for Tri6 (lane 3, Figure 10b), hygromycin (lane 7, Figure 10b) and geneticin (lane 11, Figure 10b) sequences. Notably, two PCR products with a size of 1889 bp and 672 bp were 
observed in lane 3 (Figure 10b), with the former representing the hygromycin-disrupted Tri6 and the latter representing the Tri6 from the addback plasmid. Primers used in the verification of Tri64 and Tri64-AB strains are listed in Table A4 of the Appendix. 
Figure 10: Confirmation of Tri6 disruption and complementation in F. graminearum.

(A) The Tri6 disruption construct consists of $1000 \mathrm{bp}$ of 5' and 3' element of the Tri6 coding region. This construct replaces only $485 \mathrm{bp}$ of the Tri6 coding region with hygromycin, leaving $107 \mathrm{bp}$ of the 5' end and 65 bp of the 3' end of the Tri6 ORF in the genome.

(B) PCR confirmation of Tri64 and Tri64-AB strains.

Lanes 1-4: WT, Tri64, Tri64-AB transformant \#9, water control respectively, screened with Tri6 gene specific primers, Tri6 Fsp and Tri6 End R. The PCR product at $\sim 600 \mathrm{bp}$ in lanes 1 and 3 represents the functional Tri6 gene, with an expected size is $657 \mathrm{bp}$. The PCR product at $\sim 1900$ bp in lanes 2 and 3 represents a combination of intact hygromycin gene and portions of Tri6, together with an expected size of $1889 \mathrm{bp}$.

Lanes 5-8: WT, Tri6 4 , Tri6 $4-A B$ transformant \#9, water control respectively screened with hygromycin primers, Hyg P5 and Hyg P6. The PCR product at $\sim 1800$ bp in lanes 6 and 7 represent hygromycin gene, with an expected size of $1822 \mathrm{bp}$.

Lanes 9-12: WT, Tri64, Tri64-AB transformant \#9, water control respectively screened with geneticin primers, Geneticin ENTR and Geneticin Down. The PCR product at $\sim 1200$ bp in lane 11 represents geneticin, with an expected size of $1283 \mathrm{bp}$. 
A)

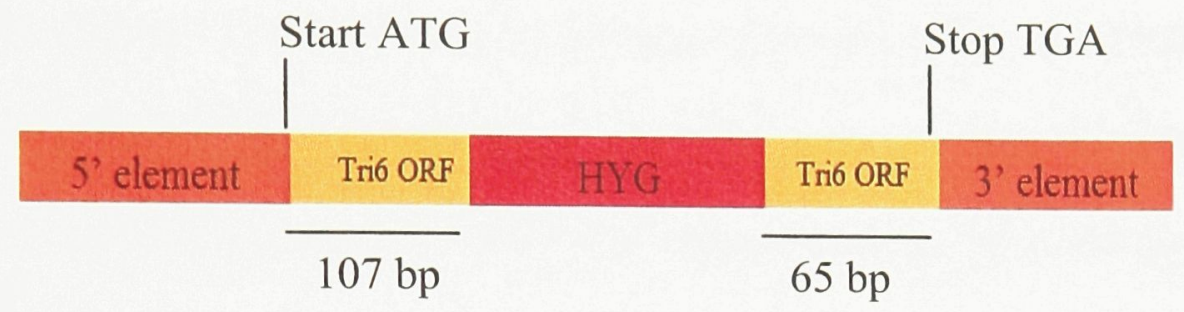

B)

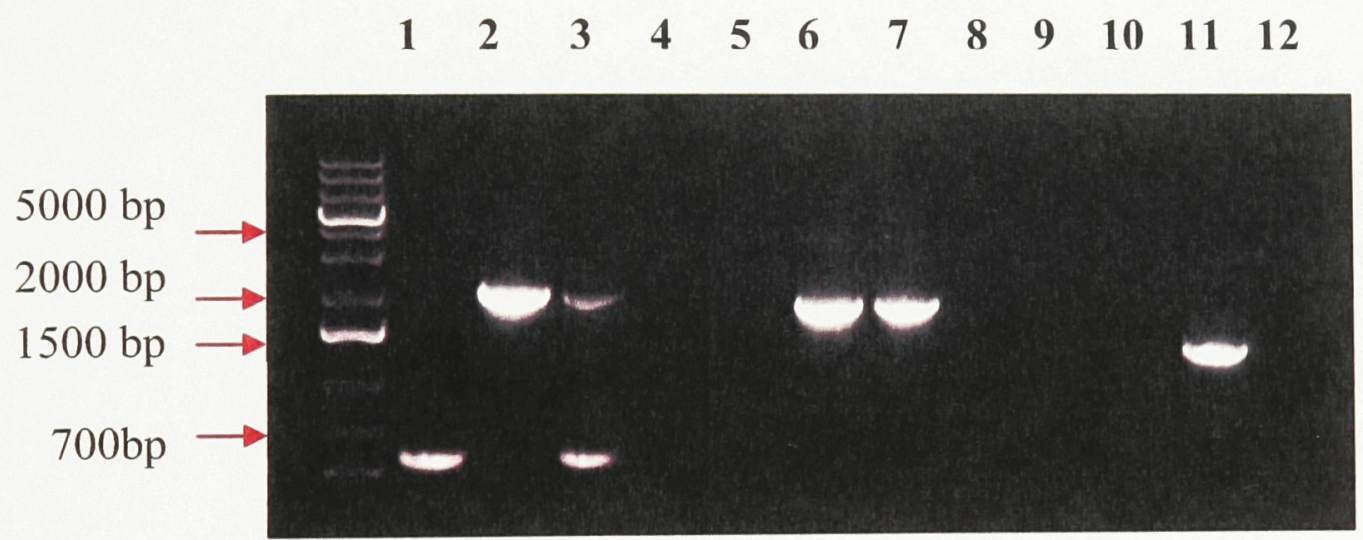




\subsubsection{Fg Tri6 is required for trichothecene production}

To determine the involvement of Tri6 in the trichothecene biosynthesis pathway of $F$. graminearum, Tri64 and Tri64-AB strains were grown under DON-inducing conditions and their filtrates were subjected to secondary metabolite analysis. As in the case of $F g$ Tri104 strain, the WT F. graminearum strain was able to synthesize the mycotoxin 15ADON (panel A, Figure 11) while the Fg Tri64 strain did not produce 15-ADON, as indicated by the absence of a 15-ADON peak (panel B, Figure 11). Complementation of Tri64 rescued its ability to produce $15-\mathrm{ADON}$, as supported by the peak eluting at 11.3 mins (panel C, Figure 11). 
Figure 11: HPLC chromatograms of $F$. graminearum Tri64 and Tri6A-AB strains cultured under DON-inducing conditions.

To determine whether Tri6 is required for trichothecene production, the media filtrate of wild type, Tri64 and Tri64-AB were subjected to HPLC analysis.

A) Chromatogram of WT F. graminearum strain 233423 grown under DON-inducing conditions. A $15-\mathrm{ADON}$ peak at 11.3 mins was observed, as indicated by the red arrow. B) Chromatogram of Fg Tri64 grown under DON-inducing conditions. The 15-ADON peak was absent, suggesting that Fg Tri64 did not produce trichothecene.

C) Chromatogram of Fg Tri64-AB grown under DON-inducing conditions. The 15ADON peak was present, as indicated by the red arrow, suggesting that the Tri6 complemented strain was able to produce trichothecene. 
A)

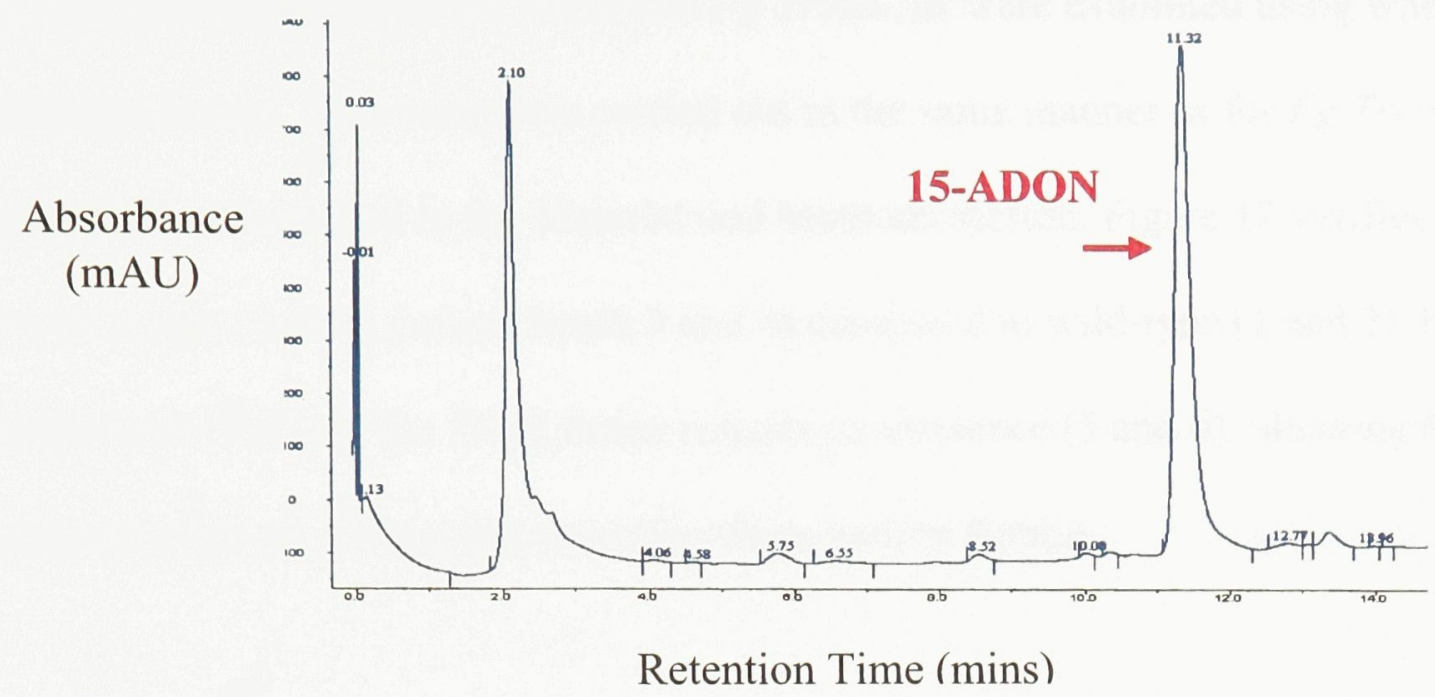

B)
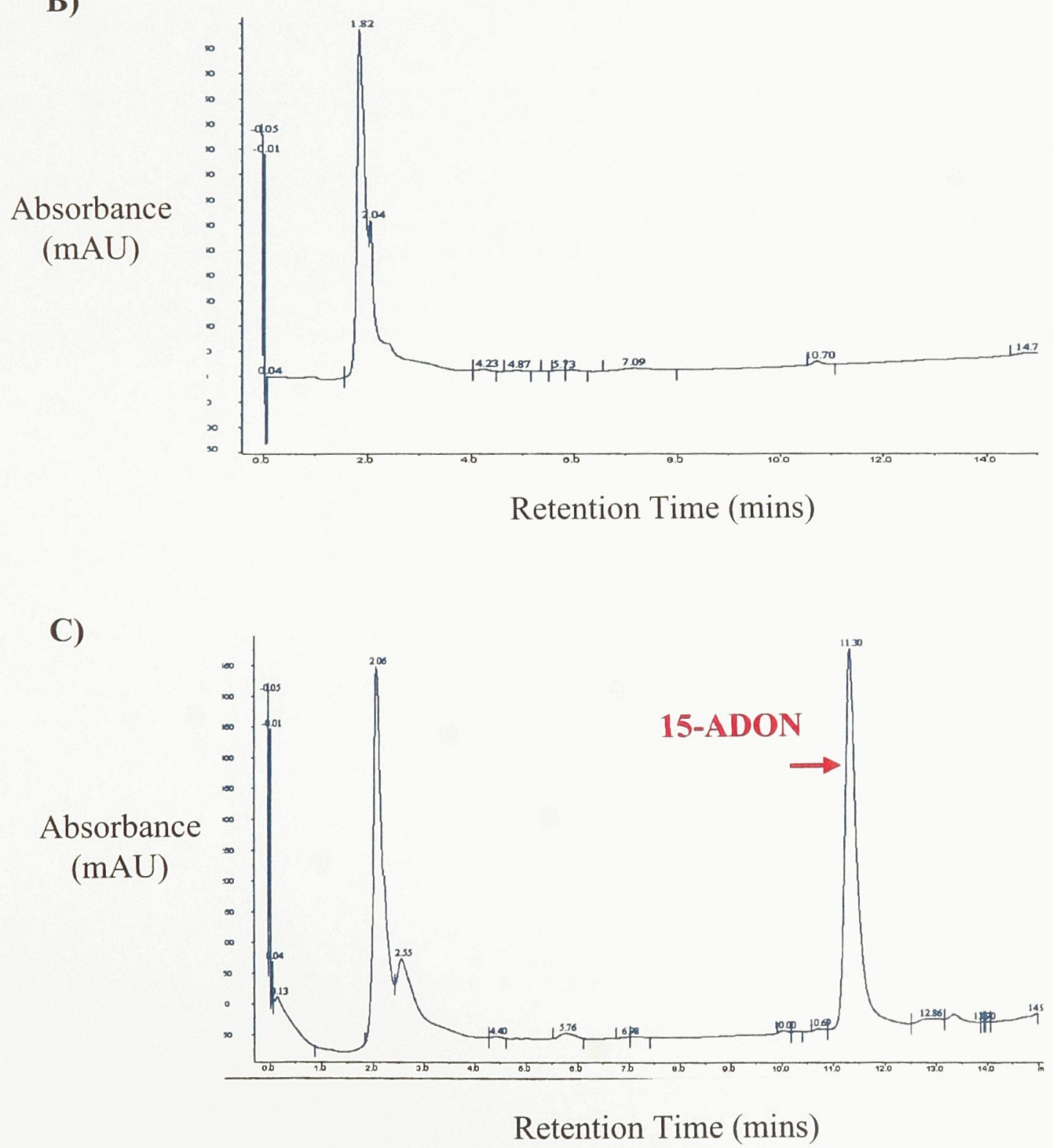


\subsubsection{Fg Tri6 contributes to virulence}

The pathogenicity of $F g$ Tri6 4 and $F g$ Tri6 6 - AB were examined using wheat infection assays. This assay was carried out in the same manner as for Fg Tri104, and the procedure is described in the Material and Methods section. Figure 12 verifies that $\mathrm{Fg}$ Tri64 is non-virulent (wheat heads 3 and 4) compared to wild-type (1 and 2), but the addition of Tri6 into the Tri6 4 strain rescues its virulence (5 and 6), allowing the addback strain to cause disease on the wheat variety Roblin. 
Figure 12: Fg Tri6 contributes to virulence in F. graminearum

Representative wheat heads (variety Roblin) after infection with WT, Tri64 and Tri64$A B$ strains.

1, 2: Wheat heads infected with $F$. graminearum WT strain. Twenty-one days subsequent to the initial inoculation, both infected wheat heads were completely bleached.

3, 4: Wheat heads infected with Fg Tri64 strain. The Tri64 strain was able to infect wheat heads but could not spread beyond the site of infection, as indicated by the browning of inoculated site only.

5, 6: Wheat heads infected with Fg Tri64-AB transformant \#9. The Tri6 complemented strain was able to infect and rescue mutant phenotype to spread beyond the site of infection, as indicated by the bleaching of the entire wheat head.

Picture was taken 21 days post infection. This experiment was repeated three times with similar results. Red arrow indicates site of inoculation. 


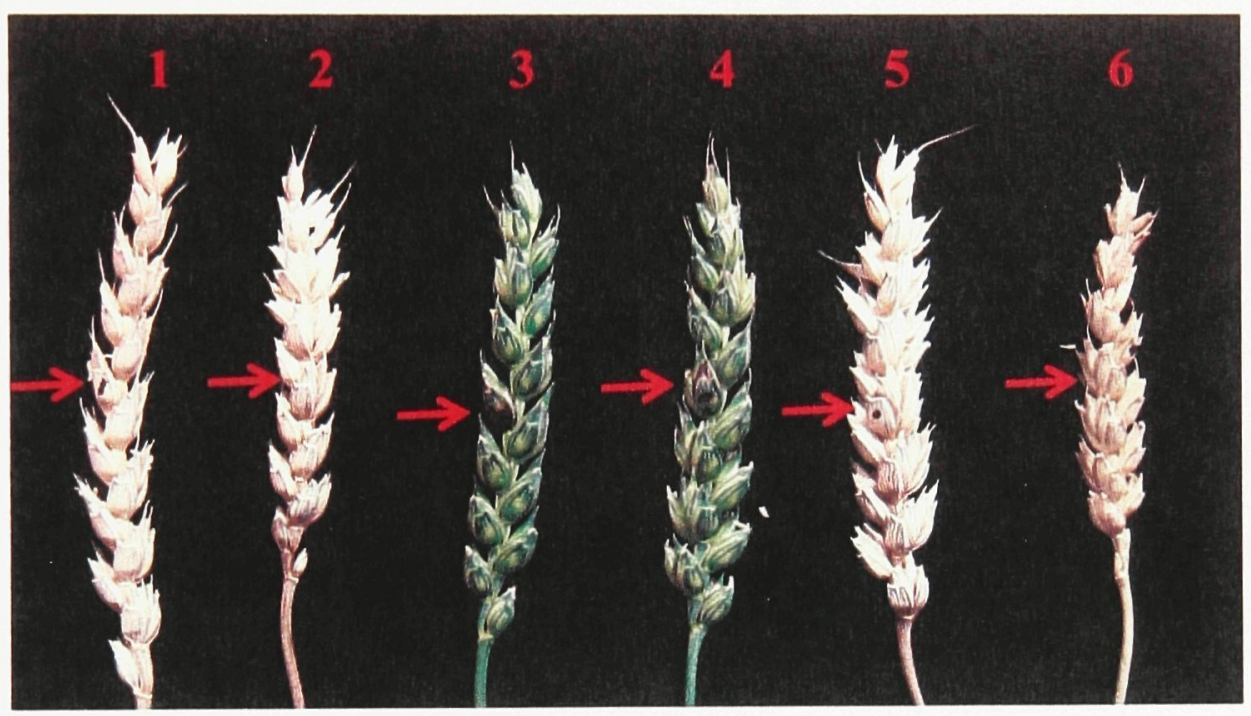




\subsection{Establishment of a Relationship between Tri10 and Tri6 in vitro}

From the previous sections, we established that both Fg Trilo and Fg Tri6 play a significant role in the trichothecene biosynthesis pathway because disruption of either gene resulted in failure to produce $15-\mathrm{ADON}$ (Figure $8 \mathrm{~B}$ and $10 \mathrm{~B}$ ). In order to identify genes that are commonly and differentially regulated by Tril0 and Tri6, we performed whole gene expression profiles on F. graminearum wild type, Tri10 $\Delta$ and Tri6 6 mutant strains grown under DON-inducing conditions. The following section is the analysis of genes that are commonly or uniquely regulated by Fg Trilo and Fg Tri6.

\subsubsection{Profiling of genes regulated by Tri104 and Tri64 under DON-inducing conditions in vitro}

Gene expression profiles were carried out using an Agilent custom made oligonucleotide microarray that consists of a total of 44, 000 oligos, of which 1,417 are controls and the rest represent 13,918 ESTs, with each gene represented by three 60 -mer oligonucleotides (Agilent Technologies Inc., Santa Clara, CA, USA). As a first step, we

were interested to know the total number of genes that are differentially expressed in the DON-inducing media. Genes expressed in WT Fusarium grown under DON noninducing conditions were compared to genes expressed in WT Fusarium grown under DON-inducing conditions. Next, gene expression profiling was performed in both Tri10 and Tri6 mutant strains and compared to the WT Fusarium, respectively, grown under DON-inducing conditions. The numbers of genes that are differentially regulated under DON-inducing conditions by these mutant strains are summarized in the Venn diagram (Figure 13). 
Figure 13: Venn diagram indicating the number of genes commonly and differentially regulated in WT, Fg Tri64, and Fg Tr104.

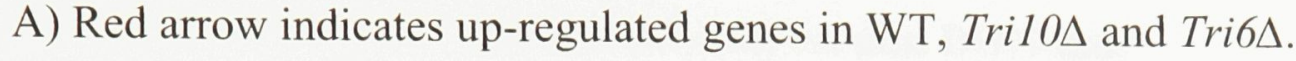

A total number of 628, 698 and 484 genes were up-regulated in wild type, Tri104, Tri64 respectively under DON-inducing conditions. Of those, 472, 417 and 151 genes were unique to the strains. The two mutant strains shared 218 commonly up-regulated genes, with Tri104 and WT sharing 41 genes, and Tri64 and WT sharing 93 genes. Together, 22 genes were up-regulated in all three strains.

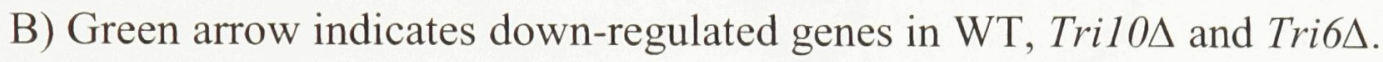

A total number of 960 and 605 genes were down-regulated in Tri10 $\Delta$ and Tri6 strains compared to wild type under DON-inducing conditions respectively, compared to only 155 down-regulated genes in the wild type under DON-inducing conditions. Of these total numbers of down-regulated genes, 87 genes were uniquely down-regulated in wild type, 484 in Tri104 strain and 164 in Tri64 strain. A total number of 412 genes were down-regulated in both Tri104 and Tri64 strains, with 39 down-regulated genes shared by Tri104 and wild type and only four by Tri64 and wild type. There were 25 genes commonly down-regulated in all three strains. 
A)

WT DON-ind. vs. non DON-ind.

Total: 628
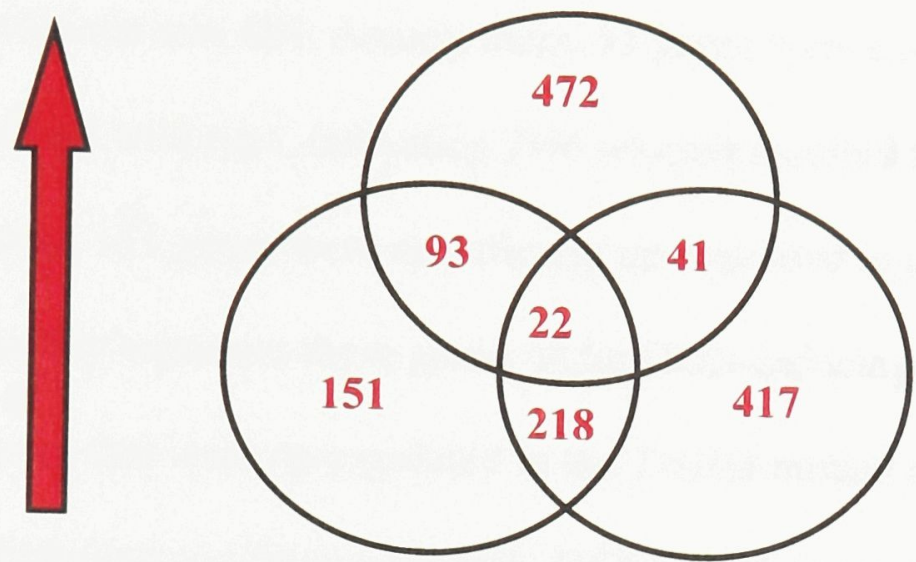

Tri64 vs. WT

Total: 484

Tri10A vs. WT

Total: 698

B)

WT DON-ind. vs. non DON-ind.

Total: 155
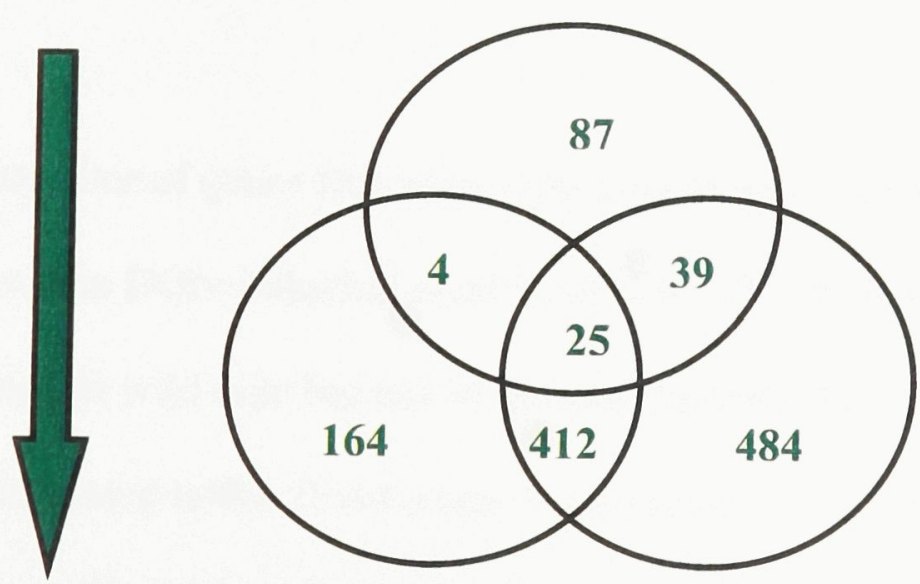

Tri6A vs. WT

Total: 605

Tri104 vs. WT

Total: 960 
The number of genes that were activated in wild type under DON-inducing conditions vs. non DON-inducing conditions was 628. Of those, 472 genes were specifically up regulated in wild type but not in the two mutants. The total number of genes that were up-regulated in the Tri64 mutant compared to wild type under DONinducing conditions was 484. Among them, 93 genes were commonly up-regulated in the Tri64 mutant and wild type, indicating Tri6 was not required for the activation of these genes. However, 151 genes were specifically up-regulated in the Tri64 strain, suggesting that Tri6 normally represses these genes under DON-inducing conditions. The total number of genes that were up-regulated in the Tri104 mutant compared to wild type under DON-inducing conditions was 698. Of those, 41 genes did not require Tri10 for activation, as they were up-regulated in both Tri104 strain and wild type. The majority, 417 genes, were up-regulated in Tril04 strain only, indicating that these genes were normally repressed in the presence of Tri10 under DON-inducing conditions. Both Tri10 and Tri6 were required for the repression of 218 genes, however, neither was responsible for the activation of 22 genes.

The number of genes that were repressed in wild type under DON-inducing conditions vs. non DON-inducing conditions was 155 . Of those, 87 genes were uniquely down-regulated in wild type but not in the two mutants. The total number of genes that were down-regulated in the Tri64 mutant compared to wild type under DON-inducing conditions was 605 . Among them, four genes were commonly down-regulated in Tri64 mutant and wild type, suggesting that Tri6 does not repress these genes. However, 164 genes were uniquely down-regulated in the Tri64 strain, suggesting that Tri6 normally 
activates their expression under DON-inducing conditions. The total number of genes that were down-regulated in the Tri10 1 mutant compared to WT under DON-inducing conditions were 960 . Of those, 39 genes were not repressed by Tri 10 under normal circumstances, as they were down-regulated in both Tri10 1 strain and wild type. 484 genes were down-regulated in Tril04 mutant only, suggesting that these genes were normally activated in the presence of Tri10 under DON-inducing conditions. Both Tri10 and Tri6 were required for the activation of 412 genes, but neither was responsible for the repression of 25 genes.

\subsubsection{Fg Tri10 and Fg Tri6 co-regulate genes involved in secondary metabolism}

Secondary metabolism refers to the synthesis of metabolites that are not essential to the growth of the organism, but contribute to their overall fitness. These pathways are typically induced under nutrient-limiting conditions or during certain developmental stages to produce and secrete toxic metabolites, hence strengthening the organism's ability to survive by eliminating nearby competitors or weakening a host plant. Biosynthetic genes involved in secondary metabolic pathways often occur in gene clusters in filamentous fungi, for example, the penicillin gene clusters in $P$. chrysogenum and A. nidulans, aflatoxin gene clusters in Aspergillus sp., and melanin gene clusters in A. alternata (Keller and Hohn, 1997). Within the Fusarium genus, some species consist of a cluster of 15 genes involved in the production of fumonisins while others contain another 15 gene-cluster encoding for genes required for the zearalenone biosynthesis pathway (Desjardins, 2006). 
Our analysis of the genes up-regulated in wild type under DON-inducing conditions but down-regulated in both Tri10 10 and Tri6 $\Delta$ mutant strains revealed regulation of two distinctive secondary metabolite clusters. The first cluster to be identified belonged to the trichothecene biosynthetic pathway leading to the production of DON and the second cluster contained genes involved in the production of butenolide (Harris et al., 2007). It is understood that Fg Tril0 and Fg Tri6 regulate the trichothecene biosynthesis pathway (Seong et al., 2009); however, regulation of the butenolide pathway was not previously known. Of the eight genes involved in the butenolide pathway, five were up-regulated in wild type under DON-inducing conditions but downregulated in both Tri104 and Tri64 mutants compared to wild type.

\subsubsection{Fg Tri10 and Fg Tri6 regulate the Tri gene cluster}

As mentioned in the Introduction, there are a total of 15 genes involved in the trichothecene biosynthesis pathway in $F$. graminearum. While 12 genes reside in the Tri gene cluster on chromosome 2, three other genes, Tril, Tri101 and Tri15, are located outside the cluster on different chromosomes (McCormick et al., 2004, Kimura et al., 1998, Desjardins, 2006). Expression levels of Tri genes in wild type and the two mutant strains can be found in Table 2. Eleven of the Tri genes were down-regulated by at least two-fold in both Tri104 and Tri64 strain, and they include Tri1 ( $\mathrm{fg} 00071)$, Tri3 (fg03534), Tri4 (fg03535), Tri5 (fg03537), Tri6 (fg03536), Tri8 (fg03532), Tri9 (fg03539), Trill (fg03540), Tri12 (fg12013), Tri14 (fg03543) and Tri101 (fg07896). It appears that Tri3 was the most strongly down-regulated Tri gene in both mutant strains, with a 100 -fold $\left(\log _{2}=-6.65\right)$ down-regulation in Tri64 and 247 -fold $\left(\log _{2}=-7.95\right)$ 
decrease in Tri104 compared to WT under DON-inducing conditions (Table 2).

Although residing outside of the Tri gene cluster, Tril was equally down-regulated as other Tri genes in both mutant strains, with a 51 -fold $\left(\log _{2}=-5.68\right)$ decrease in the Tri64 mutant and a 82 -fold $\left(\log _{2}=-6.36\right)$ decrease in the Tri104 mutant, compared to WT under DON-inducing conditions. The two Tri genes that are differentially regulated in either mutant strains are Tri10 and Tri15. Tri10 was up-regulated both in wild type by 24-fold $\left(\log _{2}=4.59\right)$ and in Tri64 strain by 4.89 -fold $\left(\log _{2}=2.29\right)$. This suggested that activation of Tri10 in DON-inducing conditions is not regulated by Tri6. Tri15 is commonly down-regulated in wild type, Tri104 and Tri64 (Table 2). In wild type grown under DON-inducing conditions, Tri15 was down-regulated by 2.5 -fold $\left(\log _{2}=-1.33\right)$, however in Tri104 mutant it was significantly down-regulated by 111 -fold $\left(\log _{2}=-6.80\right)$ and in Tri64, by 126 -fold $\left(\log _{2}=-6.98\right)$. 
Table 2: Expression levels of Tri genes in WT, Tri104 and Tri64 under DON-inducing conditions

\begin{tabular}{|c|c|c|c|c|c|}
\hline $\begin{array}{c}\text { Fg } \\
\text { Number }\end{array}$ & Tri gene & $\begin{array}{c}\text { Annotated } \\
\text { Function/Description }\end{array}$ & $\begin{array}{c}\text { Tri64 vs } \\
\text { WT } \\
\text { (DON- } \\
\text { inducing } \\
\text { conditions) } \\
*\end{array}$ & $\begin{array}{c}\text { Tril04 vs } \\
\text { WT } \\
\text { (DON- } \\
\text { inducing } \\
\text { conditions) } \\
*\end{array}$ & $\begin{array}{c}\text { WT DON- } \\
\text { inducing } \\
\text { vs WT non } \\
\text { DON } \\
\text { inducing } \\
\text { conditions } \\
*\end{array}$ \\
\hline fg00071 & Tril & $\begin{array}{c}\text { cytochrome } \mathrm{P} 450 \\
\text { oxygenase }\end{array}$ & $\begin{array}{c}-5.68+/- \\
1.64\end{array}$ & $\begin{array}{c}-6.36+/- \\
1.64\end{array}$ & $\begin{array}{c}5.28+/- \\
0.55\end{array}$ \\
\hline fg03534 & Tri3 & $\begin{array}{l}\text { trichothecene } 15-\mathrm{O}- \\
\text { acetyltransferase }\end{array}$ & $\begin{array}{c}-6.65+/- \\
0.53\end{array}$ & $\begin{array}{l}-7.95+/- \\
1.09\end{array}$ & $\begin{array}{c}6.32+/- \\
0.27\end{array}$ \\
\hline fg03535 & Tri4 & $\begin{array}{l}\text { trichodiene oxygenase } \\
\text { [cytochrome P450] }\end{array}$ & $\begin{array}{c}-2.02+/- \\
0.21\end{array}$ & $\begin{array}{c}-6.45+/- \\
0.50\end{array}$ & $\begin{array}{c}5.26+/- \\
0.48\end{array}$ \\
\hline fg03537 & Tri5 & $\begin{array}{l}\text { trichodiene synthase } \\
\text { [sesquiterpene cyclase] }\end{array}$ & $\begin{array}{c}-5.91+/- \\
0.61\end{array}$ & $\begin{array}{c}-6.40+/- \\
1.05\end{array}$ & $\begin{array}{c}5.40+/- \\
0.25\end{array}$ \\
\hline fg03536 & Tri6 & $\begin{array}{l}\text { trichothecene } \\
\text { biosynthesis positive } \\
\text { transcription factor }\end{array}$ & $\begin{array}{c}-3.30+/- \\
0.77\end{array}$ & $\begin{array}{c}-3.95+/- \\
0.55\end{array}$ & $\begin{array}{c}3.64+/- \\
0.04\end{array}$ \\
\hline fg03532 & Tri8 & $\begin{array}{l}\text { trichothecene 3-O- } \\
\text { esterase }\end{array}$ & $\begin{array}{c}-5.06+/- \\
0.15 \\
\end{array}$ & $\begin{array}{c}-6.34+/- \\
0.52 \\
\end{array}$ & $\begin{array}{c}2.44+/- \\
0.14 \\
\end{array}$ \\
\hline fg03539 & Tri9 & hypothetical protein & $\begin{array}{c}-3.57+/- \\
0.11\end{array}$ & $\begin{array}{c}-5.50+/- \\
0.13\end{array}$ & $\begin{array}{c}2.67+/- \\
0.16\end{array}$ \\
\hline fg03538 & Trilo & regulatory protein & $\begin{array}{c}2.29+/- \\
0.06\end{array}$ & $\begin{array}{l}-4.69+/- \\
0.50\end{array}$ & $\begin{array}{l}4.59+/- \\
0.05\end{array}$ \\
\hline fg03540 & Trill & $\begin{array}{l}\text { isotrichodermin C-15 } \\
\text { hydroxylase }\end{array}$ & $\begin{array}{l}-4.35+/- \\
0.06\end{array}$ & $\begin{array}{l}-7.10+/- \\
0.59 \\
\end{array}$ & $\begin{array}{c}4.86+/- \\
0.95\end{array}$ \\
\hline fg12013 & Tril2 & $\begin{array}{l}\text { trichothecene efflux } \\
\text { pump }\end{array}$ & $\begin{array}{l}-4.26+/- \\
0.11\end{array}$ & $\begin{array}{l}-6.85+/- \\
0.56\end{array}$ & $\begin{array}{c}3.09+/- \\
0.09\end{array}$ \\
\hline fg03543 & Tril4 & $\begin{array}{l}\text { putative trichothecene } \\
\text { biosynthesis gene }\end{array}$ & $\begin{array}{l}-5.63+/- \\
0.56 \\
\end{array}$ & $\begin{array}{c}-6.80+/- \\
0.54\end{array}$ & $\begin{array}{c}4.44+/- \\
0.12\end{array}$ \\
\hline fgl1025 & Tril5 & $\begin{array}{l}\text { putative } \mathrm{C} 2 \mathrm{H} 2 \text { zinc } \\
\text { finger transcription } \\
\text { factor }\end{array}$ & $\begin{array}{l}-6.98+/- \\
1.26\end{array}$ & $\begin{array}{c}-6.80+/- \\
0.48\end{array}$ & $\begin{array}{l}-1.33+/- \\
0.14\end{array}$ \\
\hline fg07 & Tril01 & $\begin{array}{l}\text { trichothecene 3-O- } \\
\text { acetyltransferase }\end{array}$ & $\begin{array}{l}-5.28+/- \\
0.24\end{array}$ & $\begin{array}{c}-5.14+/- \\
0.22\end{array}$ & $\begin{array}{l}0.05+/- \\
0.01\end{array}$ \\
\hline
\end{tabular}

\footnotetext{
* value represented as $\log _{2}$.
} 


\subsubsection{Fg Trilo and Fg Tri6 regulate the butenolide pathway in vitro}

The butenolide gene cluster spans $17 \mathrm{~kb}$ on chromosome 2 of $F$. graminearum and codes for eight genes required for the butenolide biosynthesis pathway (Harris et al., 2007). These eight genes, $f g 08077$ - $f g 08084$, synthesize a secondary metabolite known as butenolide, which has been associated with the fescue foot syndrome in cattle.

Affected cattle experience swelling and lesions in their hooves, weight loss and in severe cases, the loss of feet (Bacon, 1995). Previous secondary metabolite analysis using HPLC indicated the secretion of both $15-\mathrm{ADON}$ and butenolide by $F$. graminearum into the medium under DON-inducing conditions (Harris et al., 2007). Our microarray data suggests that along with the Tri gene cluster, Tri10 and Tri6 also regulate the butenolide gene cluster in wild type during DON-inducing conditions. According to Table 3, six genes from the butenolide gene cluster, $f g 08077$ and $f g 08079$ - $f g 08083$, were downregulated in both Tri104 and Tri64 mutants. For example, $f g 08079$ codes for a benzoate 4-monooxygenase cytochrome P450 that was up-regulated in wild type under DONinducing conditions by 7.31 -fold $\left(\log _{2}=2.87\right)$ and down-regulated in Tri104 and Tri64 by 48 -fold $\left(\log _{2}=-5.59\right)$ and 16 -fold $\left(\log _{2}=-4.02\right)$, respectively. Fg08078 was not down-regulated in Tri64 by two-fold, and expression levels of fg08084 were not available. 
Table 3: Expression levels of genes involved in the butenolide biosynthetic pathway under DON-inducing conditions

\begin{tabular}{|c|c|c|c|c|}
\hline Fg Number & $\begin{array}{c}\text { Annotated } \\
\text { Function/Description }\end{array}$ & $\begin{array}{c}\text { Tri64 vs WT } \\
\text { (DON- } \\
\text { inducing } \\
\text { conditions)* }\end{array}$ & $\begin{array}{c}\text { Tri104 vs } \\
\text { WT } \\
\text { (DON- } \\
\text { inducing } \\
\text { conditions)* }\end{array}$ & $\begin{array}{c}\text { WT DON- } \\
\text { inducing vs } \\
\text { WT non } \\
\text { DON } \\
\text { inducing } \\
\text { conditions * }\end{array}$ \\
\hline fg08077 & $\begin{array}{c}\text { related to flavin } \\
\text { oxidoreductase }\end{array}$ & $-1.59+/-0.15$ & $-2.68+/-0.39$ & $0.52+/-0.08$ \\
\hline fg08078 & $\begin{array}{c}\text { related to general } \\
\text { amidase }\end{array}$ & $-0.90+/-0.04$ & $-2.03+/-0.07$ & $0.96+/-0.03$ \\
\hline fg08079 & $\begin{array}{c}\text { probable benzoate } 4- \\
\text { monooxygenase } \\
\text { cytochrome P450 }\end{array}$ & $-4.02+/-0.54$ & $-5.59+/-0.91$ & $2.87+/-0.58$ \\
\hline fg08080 & $\begin{array}{c}\text { conserved } \\
\text { hypothetical protein }\end{array}$ & $-1.10+/-0.07$ & $-3.54+/-0.26$ & $1.93+/-0.00$ \\
\hline fg08081 & $\begin{array}{c}\text { related to gibberellin } \\
20-\text {-oxidase }\end{array}$ & $-1.64+/-0.09$ & $-5.00+/-0.83$ & $3.63+/-0.56$ \\
\hline fg08082 & $\begin{array}{c}\text { conserved } \\
\text { hypothetical protein }\end{array}$ & $-1.68+/-0.06$ & $-5.04+/-0.17$ & $3.23+/-0.15$ \\
\hline fg08083 & $\begin{array}{c}\text { related to glutamic } \\
\text { acid decarboxylase }\end{array}$ & $-2.27+/-0.12$ & $-4.80+/-1.18$ & $2.53+/-0.82$ \\
\hline fg08084 & \multicolumn{2}{|c|}{ N/A } \\
\hline
\end{tabular}

* value represented as $\log _{2}$. 


\subsubsection{Fg Tri10 and Fg Tri6 regulate cytochrome P450 oxygenases in vitro}

Cytochrome P450 oxygenases are a superfamily of heme-containing enzymes that introduce oxygen into substrate molecules, thus generating a wide range of reactions. In the trichothecene biosynthesis pathway, these enzymes typically hydroxylate a particular carbon on the trichothecene backbone. Four Tri genes code for cytochrome P450 oxygenases: Tri1 hydroxylates carbon 8 in type B trichothecenes to produce 8hydroxycalonectrin (Figure 14), Tri4 hydroxylates multiple carbons, while Tril1 hydroxylates carbon 15, and finally Tri 13 hydroxylates carbon 4 of trichothecene only in the NIV-producing Fg strains. 
Figure 14: Potential action of a cytochrome P450 oxygenase in the trichothecene biosynthesis pathway. (Image taken from McCormick et al., 2004).

In this selected reaction from the trichothecene biosynthesis pathway, Tril gene product hydroxylates carbon 8 of calonectrin to produce 8-hydroxycalonectrin. However, the enzyme driving the subsequent hydroxylation reaction from 8-hydroxycalonectrin to 7,8dihydroxylcalonectrin has not been identified. 


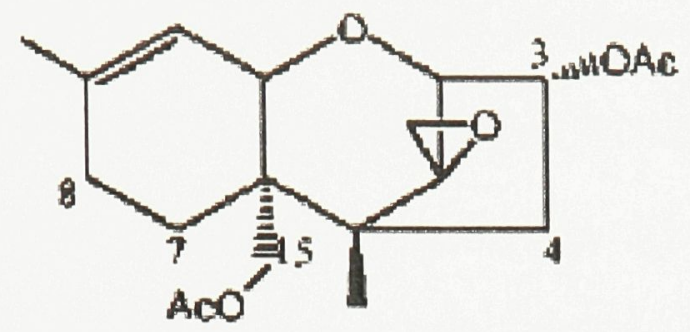

Calonectrin

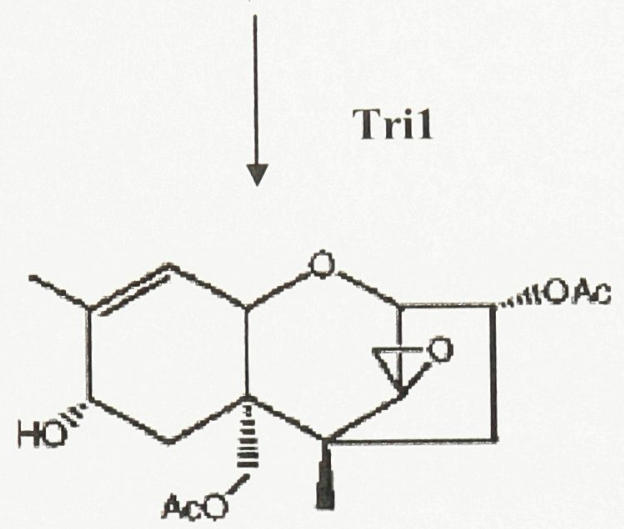

8-hydroxycalonectrin

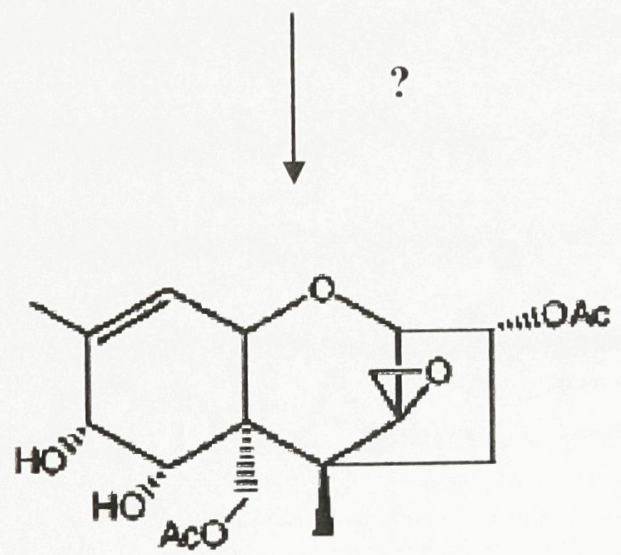

7, 8-dihydroxycalonectrin 
In addition to regulating the three oxygenase-encoding Tri genes (Tril, Tri4 and Trill) described above, Tri10 and Tri6 also regulate three additional cytochrome P450 oxygenases, namely, $f g 00007, f g 08023$ and $f g d 224-50$. Since not all the enzymes catalyzing the trichothecene biosynthesis pathway have been characterized, these oxygenases could be potential candidates in that pathway. Also, cytochrome P450s have been documented to detoxify phytoalexins to promote fitness of the organism, and it is possible these three cytochrome P450s function to promote fitness of the fungus by way of this.

Of the three oxygenases, the expression level of $f g 00007$ was the most reduced in Tri104 and Tri64. This gene was down-regulated by 191 -fold $\left(\log _{2}=-7.58\right)$ in Tri104 and by 108 -fold $\left(\log _{2}=-6.76\right)$ in Tri64 mutant strains (Table 4). The second P450 gene, fg08023, was up-regulated by 2 -fold $\left(\log _{2}=1.03\right)$ in WT, but down-regulated by 26.7 fold $\left(\log _{2}=-4.74\right)$ in Tri104 and by 12.7 -fold $\left(\log _{2}=-3.67\right)$ in Tri64 strains. The third gene, $F g d 224-50$, increased in expression by 2 -fold $\left(\log _{2}=1.03\right)$ in WT grown under DON-inducing conditions, but was decreased in expression by 2.9-fold $\left(\log _{2}=-1.55\right)$ in Tri104 and by 2.4 -fold $\left(\log _{2}=-1.25\right)$ in Tri64 when compared to WT. 
Table 4: Expression levels of genes encoding for cytochrome P450 oxygenases regulated by Tril0 and Tri6 under DON-inducing conditions

\begin{tabular}{|c|c|c|c|c|}
\hline Fg Number & $\begin{array}{c}\text { Annotated } \\
\text { Function/Description }\end{array}$ & $\begin{array}{c}\text { Tri64 vs WT } \\
\text { (DON- } \\
\text { inducing } \\
\text { conditions)* }\end{array}$ & $\begin{array}{c}\text { Tri104 vs } \\
\text { WT } \\
\text { (DON- } \\
\text { inducing } \\
\text { conditions)* }\end{array}$ & $\begin{array}{c}\text { WT DON- } \\
\text { inducing vs } \\
\text { WT non } \\
\text { DON } \\
\text { inducing } \\
\text { conditions * }\end{array}$ \\
\hline $\begin{array}{c}\text { fg00071 } \\
(\text { Tri } 1)\end{array}$ & $\begin{array}{c}\text { cytochrome P450 } \\
\text { oxygenase }\end{array}$ & $-5.68+/-1.64$ & $-6.36+/-1.64$ & $5.28+/-0.55$ \\
\hline $\begin{array}{c}\text { fg03535 } \\
(\text { Tri4 })\end{array}$ & $\begin{array}{c}\text { trichodiene oxygenase } \\
\text { (cytochrome P450] }\end{array}$ & $-2.02+/-0.21$ & $-6.45+/-0.50$ & $5.26+/-0.48$ \\
\hline $\begin{array}{c}\text { fg03540 } \\
(\text { Trill })\end{array}$ & $\begin{array}{c}\text { isotrichodermin C-15 } \\
\text { hydroxylase }\end{array}$ & $-4.35+/-0.06$ & $-7.10+/-0.59$ & $4.86+/-0.95$ \\
\hline fg00007 & $\begin{array}{c}\text { related to O- } \\
\text { methylsterigmatocystin } \\
\text { oxidoreductase }\end{array}$ & $-6.76+/-0.16$ & $-7.58+/-0.24$ & $2.71+/-0.43$ \\
\hline fg08023 & $\begin{array}{c}\text { related to } \\
\text { isotrichodermin C-15 } \\
\text { hydroxylase } \\
\text { (cytochrome P-450 } \\
\text { monooxygenase } \\
\text { CYP65A1) }\end{array}$ & $-3.67+/-0.14$ & $-4.74+/-0.20$ & $1.03+/-0.03$ \\
\hline & $\begin{array}{c}\text { related to benzoate } 4- \\
\text { monooxygenase } \\
\text { cytochrome P450 }\end{array}$ & $-1.25+/-0.05$ & $-1.55+/-0.06$ & $1.03+/-0.00$ \\
\hline fgd224-50
\end{tabular}

* value represented as $\log _{2}$. 


\subsubsection{Differential regulation of genes by Tri10 and Tri6}

Our analysis thus far has focused on genes that are co-regulated by both Tril0 and Tri6. In order to identify genes up-regulated in wild type during DON-inducing conditions that are regulated uniquely by either Tri10 or Tri6, two comparisons were carried out. The first comparison was between the 472 genes uniquely up-regulated in wild type to the 484 down-regulated genes specific to Tri10 10 mutant, and the second comparison was between the same 472 up-regulated in wild type and the 164 downregulated genes specific to the Tri6 $\Delta$ mutant strain. Our analysis revealed that while there were 180 genes uniquely regulated by Tri10 (Table A21) under DON-inducing conditions in vitro, only ten genes could be associated with Tri6 regulation (Table A22). With the significant difference in the number of affected genes, we were interested to know the functional category of genes that are specifically influenced by Tri10 and Tri6. As displayed in Table 5, among the annotated genes, the major category regulated by Tri10 or Tri6 belongs to the genes regulating cellular metabolism, specifically genes involved in carbon and nitrogen metabolism. However, the data also revealed that Tri10 exerts its influence on genes involved in cellular transport, especially in ion transport, as well as genes involved in defense and virulence. This categorization supports the fact that Tri10 and Tri6 play differential roles outside of the trichothecene biosynthesis pathway. 
Table 5: MIPS Functional category* of genes potentially activated by Tri10 or Tri6 under DON-inducing conditions

\begin{tabular}{|c|c|c|}
\hline Functional Category & $\begin{array}{l}\text { Genes potentially } \\
\text { activated by Trilo }\end{array}$ & $\begin{array}{l}\text { Genes potentially } \\
\text { activated by Tri6 }\end{array}$ \\
\hline Metabolism & 45 & 6 \\
\hline Energy & 9 & 0 \\
\hline $\begin{array}{l}\text { Cell cycle and DNA } \\
\text { processing }\end{array}$ & 3 & 1 \\
\hline Transcription & 1 & 0 \\
\hline $\begin{array}{l}\text { Protein fate (folding, } \\
\text { modification, destination) }\end{array}$ & 6 & 2 \\
\hline $\begin{array}{l}\text { Protein with binding function } \\
\text { or co-factor requirement }\end{array}$ & 11 & 1 \\
\hline Cellular transport & 18 & 1 \\
\hline $\begin{array}{l}\text { Cell rescue, defense and } \\
\text { virulence }\end{array}$ & 17 & 2 \\
\hline $\begin{array}{l}\text { Systemic interaction with } \\
\text { environment }\end{array}$ & 1 & 2 \\
\hline $\begin{array}{l}\text { Cell fate and cell type } \\
\text { differentiation }\end{array}$ & 4 & 0 \\
\hline $\begin{array}{l}\text { Biogenesis of cellular } \\
\text { components }\end{array}$ & 6 & 1 \\
\hline $\begin{array}{l}\text { Regulation of metabolism } \\
\text { and protein function }\end{array}$ & 0 & 1 \\
\hline $\begin{array}{l}\text { Cellular communication/ } \\
\text { Signal transduction } \\
\text { mechanism }\end{array}$ & 0 & 2 \\
\hline Unclassified & 112 & 4 \\
\hline
\end{tabular}

* MIPS = Munich information center for protein sequences (http://mips.helmholtzmuenchen.de/genre/proj/fusarium) 


\subsection{Confirmation of Microarray Data by RNA Blot Analysis of Selected Genes}

Northern analysis was performed on RNA samples extracted from WT, Tril0 deleted mutant (Tri104), Tril0 complemented (Tri104-AB), Tri6 deleted mutant (Tri64), and Tri6 complemented (Tri6 $\triangle-A B$ ) strains of $F$. graminearum harvested after 24 hours incubation in first stage non DON-inducing media and 12 hours in second stage DONinducing media. Figure 15 shows gene expression of the four genes, Tri1, Fg00007, Tri6, and Tri10, grown in non DON-inducing conditions (panel A) and DON-inducing conditions (panel B). While Tril, Fg00007 and Tri10 probes span the entire coding region of the genes, the Tri6 probe only spans 562 bp of the Tri6 coding region beginning at the $86^{\text {th }}$ bp from the ATG start site.

As demonstrated in Figure 15, the two P450 genes, Tril and fg00007, exhibited similar profiles. Both are induced under DON-inducing conditions in the wild type (compare panel A and B, Tril and fg00007, Figure 15) and similarly both are downregulated in the Tri10 and Tri6 mutant strains (lanes 2 and 4, Tril and fg00007, panel B, Figure 15). Gene expression of Tril and $f g 00007$ were recovered to wild type levels in both complemented strains, Tri10 $-A B$ and Tri6 $4-A B$ (compare lanes 1, 3 and 5, Tril and fg00007, panel B, Figure 15).

Northern analysis confirmed that Tril0 expression was strongly induced under nutrient limiting conditions (lane 1, Tri10, panel B, Figure 15). Tri10 expression was equally induced in the Tri64 mutant strain. This data, corroborated by our microarray analysis, strongly indicated that activation of Tri10 was not dependent on the presence of 
Tri6 gene. Our analysis of Tri6 expression was contradictory to the data obtained from our microarray analysis. The microarray analysis (Table 2) suggested that Tri6 expression was down regulated in the Tri10 10 mutant strain, however, Northern analysis indicated that expression of Tri6 is unaffected by the absence of Tri10 (lane 4, Tri6, panel B, Figure 15).

Although Northern analysis showed that Tri6 expression was significantly upregulated in nutrient-limiting conditions with RNA corresponding to the correct size (white arrow, lane 1, Tri6, panel B, Figure 15), we also observed that in the Tri64 mutant strain, a new RNA species with a high molecular weight strongly hybridized to the Tri6 probe (red arrow, lane 2 and 3, Tri6, panel B, Figure 15). Since the Tri6 probe overlaps with the portion of Tri6 that was not replaced by Hygromycin, as indicated by the red line in Figure 10a, we speculate that this is a transcript read through into the Hygromycin gene. More significantly, the Tri6 gene is apparently induced in the Tri64 mutant strain even in non DON-inducing media (lane 2, Tri6, panel A, Figure 15). Therefore, this suggests that the expression of Tri6 is negatively regulated by its own product. Given the fact that there are two putative binding sites of Tri6 in its own promoter, this is a likely scenario. 
Figure 15: Gene expressions of Tril, Fg00007, Tril0 and Tri6 under non DON-inducing conditions and DON-inducing conditions.

Lanes 1-5 represents $10 \mu \mathrm{g}$ of total RNA extracted from WT, Tri6 6 , Tri6 $\Delta-A B, \operatorname{Tri} 10 \Delta$, Tri10 $\triangle-A B$ strains of $F$. graminearum, respectively. Equal loading of RNA was monitored by ethidium bromide straining adjacent to the RNA blot. Panel A represents expression profiles of Tril, Fg00007, Tril0 and Tri6 under non DON-inducing conditions. Under these conditions, neither Tril nor Fg00007 were

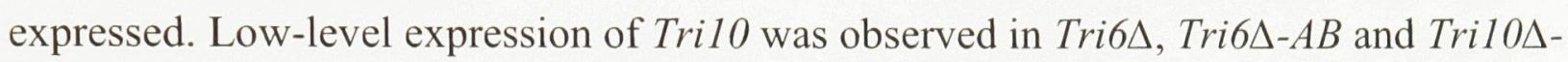
$A B$ (lanes 2, 3 and 5). High-level expression of Tri6 was observed in Tri6 $\triangle$ and Tri6 $\Delta-A B$ (lanes 2 and 3).

Panel B represents expression profiles of Tril, Fg00007, Tril0 and Tri6 under DONinducing conditions. Under these conditions, both Tril and Fg00007 were not expressed

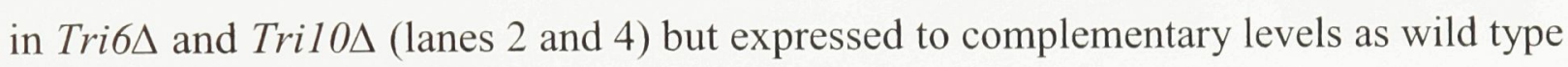
in Tri6 $\triangle-A B$ and Tri10 $\triangle-A B$ strains (lanes 1,3 and 5). Under DON-inducing conditions, Tril0 was expressed to high levels in WT, Tri6 6 and both addback strains (lanes 1, 2, 3 and 5). However, its expression was absent in Tri10ム. Under similar conditions, Tri6 was highly expressed in WT, Tri10 $\Delta$ and the two addback strains (white arrow, lanes 1, 3, 4 and 5). However, in Tri6 $\Delta-A B$ strain, an additional species was observed (red arrow, lane 3). This higher molecular weight RNA species is also observed in Tri6 $\Delta$ strain (lane 2). This likely represents the transcript read-through of the hygromycin gene initiating from the Tri6 ATG start codon. 


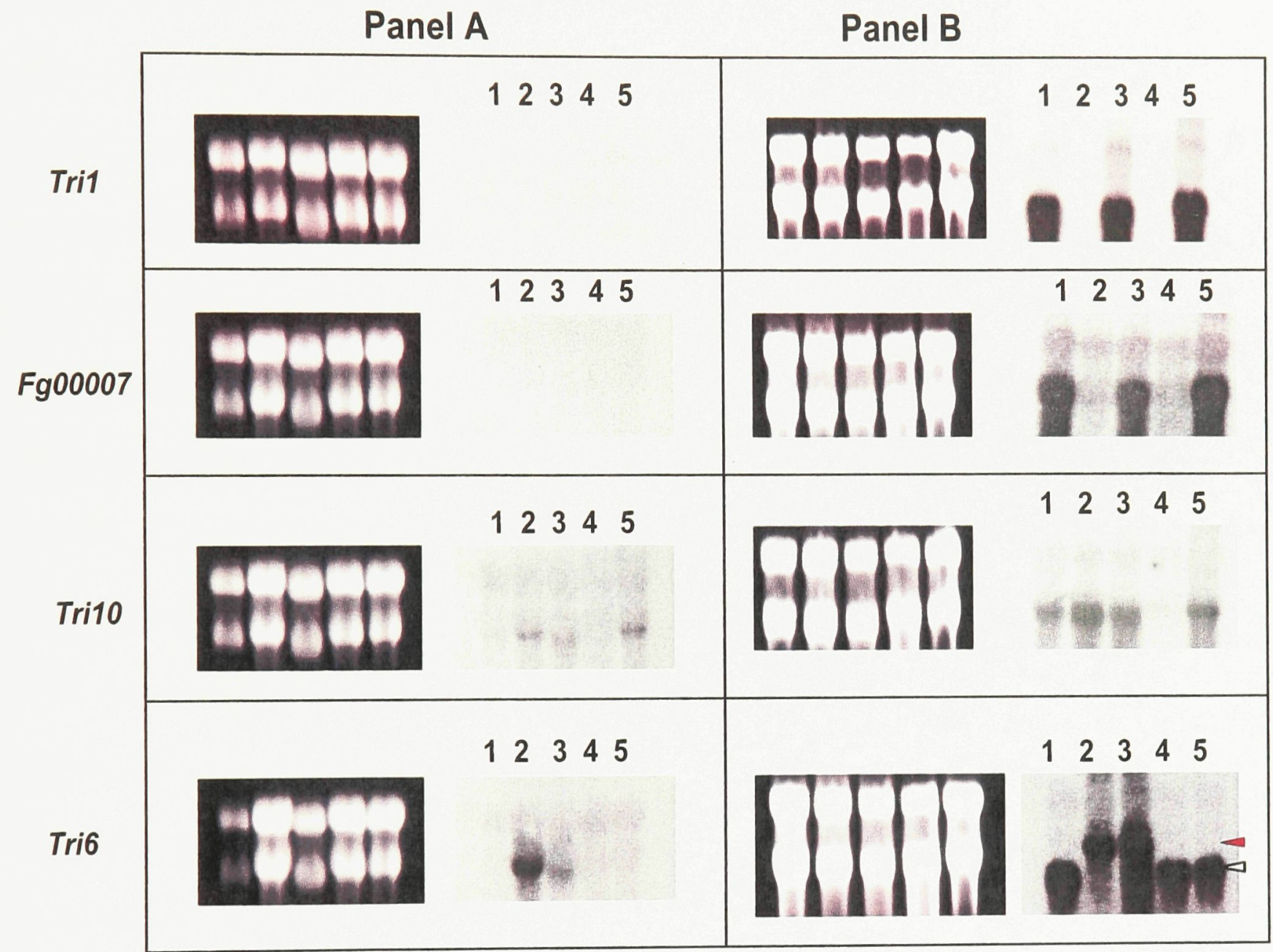




\section{Chapter 4 - Discussion}

Fusarium graminearum is a remarkably successful fungal pathogen. The pathogen colonizes a wide host range encompassing many habitats. One component of its success could be attributed to its ability to produce a wide range of secondary metabolites, many of whose functions are uncharacterized. One of the well characterized secondary metabolites are the trichothecenes. With inhibitory effects on protein synthesis, it compromises the host's defense system, thus providing the fungus with an advantage in the host -pathogen battle. Although trichothecenes play such an important role in the pathogenicity of $F$. graminearum, their activation and regulation is not fully understood. Many past studies have demonstrated that Tri10 and Tri6 act as positive regulators of the mycotoxin production pathway and up-regulate genes involved in the trichothecene pathway under nutrient-limiting conditions (Proctor et al., 1995, Tag et al., 2001, Peplow et al., 2003, Seong et. al., 2009). The existing model suggests that regulation of the trichothecene biosynthesis pathway occurs in a linear fashion, where Tri10 regulates Tri6, which in turn activates other Tri genes (Tag et al., 2001). In contrast, this study revealed that the regulation of Tri10 and Tri6 is independent of each other. This is also supported by gene expression profiling data performed on infected wheat heads by the same mutants (Seong et al., 2009). Our microarray analysis has provided evidence that the regulatory roles of Tri10 and Tri6 in F. graminearum extend their control beyond the trichothecene biosynthetic pathway. The analysis showed that another secondary metabolic cluster involved in the production of butenolide is also co-regulated by Tri10 and Tri6. Finally, coordinated expression of genes involved in primary metabolism by $\mathrm{Fg}$ 
TrilO and Fg Tri6 identifies a regulatory network that links these primary and secondary metabolic pathways.

\subsection{Coordinated and non-coordinated expression of Tri genes by Tri10 and Tri6}

Our microarray data indicated that eleven out of the thirteen functional Tri genes (Tri1, 3, 4, 5, 6, 8, 9, 11, 12, 14, 101) are regulated by both Tri10 and Tri6 in DONproducing $F$. graminearum. The two Tri genes that are differentially regulated in Tri10د and Tri64 mutant strains are Tril5 and Tri10. The expression of Tril5 was decreased by 2.5-fold ( $\left.\log _{2}=-1.33\right)$ in wild type under DON-inducing conditions, indicating it might not be required for the production of trichothecenes. In the sister species $F$. sporotrichioides, Tri 15 codes for a $\mathrm{Cys}_{2} \mathrm{His}_{2}$ zinc finger protein and was described as a negative regulator of the pathway based on the fact that this gene was strongly expressed when the fungal culture was treated with the end product T2-toxin (Alexander et al., 2004). However, the disruption of this gene did not affect trichothecene production (Alexander et al., 2004). Similarly, disruption of this gene in F. graminearum did not affect the production of 15-ADON in culture (unpublished results). Our microarray analysis showed that Fg Tril5 is positively regulated by both TrilO and Tri6, as its expression was down regulated by 111 -fold $\left(\log _{2}=-6.80\right)$ in Tri10 4 strain and 125 -fold $\left(\log _{2}=-6.98\right)$ in Tri6 4 strain. Currently, the role of Tril5 in the trichothecene pathway is unclear. Since the expression pattern of the two secondary metabolic pathways (trichothecene and butenolide) are co-regulated by Tril0 and Tri6, it would be worthwhile examining the expression of Tri15 in a F. graminearum mutant strain that does not produce butenolide. 
In contrast to the expression patterns of most Tri genes, Tril0 expression is not typical. The expression of Tril0 is induced in the Tri64 mutant by 4.89 -fold $\left(\log _{2}=\right.$ 2.29). This was confirmed by the RNA blot analysis, where we showed that Trilo was expressed in low amounts in Tri64, Tri64-AB and Tri10 $-A B$ strains under non DONinducing conditions (lanes 2 and 3, Tri10, panel A, Figure 15). Under DON-inducing conditions, Tril0 transcripts accumulated to higher levels in the Tri64, suggesting that Tri6 may act as a negative regulator of Tri10. This idea is supported by the data obtained in F. sporotrichioides, where deletion of Tri6 also resulted in the over-expression of Tril0 (Tag et al., 2001). Since the promoter of Tril0 does not possess the Tri6 binding site, the regulation of Tril0 by Tri6 is likely indirect.

Microarray analysis also indicated that the expression of Tri6 was down regulated in the Tril0 $\Delta$ mutant strain, thus implicating the requirement of Tril0 for Tri6 activation. This observation corresponds to the regulatory model of trichothecene biosynthesis pathway in F. sporotrichioides proposed by Tag et al. (2001), where Tri10 acts upstream of Tri6. However, our RNA blot analysis does not agree with this linear regulation of Tri6 by Tri10. Tri6 transcripts accumulated to comparable amounts in both the wild type strain and Tri10 mutant strain (lanes 1 and 4, Tri6, panel B, Figure 15), implying that activation of Tri6 expression does not require Tri10. The discrepancy between results generated from microarray and Northern analysis suggests a temporal regulation of this gene. The microarray was performed with RNA extracted after four hours under DONinducing conditions, whereas the Northern analysis was performed with RNA extracted after twelve hours under the same conditions. Recent results from our laboratory 
(Subramaniam, personal communications) indicate a complex auto-regulation of Tri6 expression. This is borne out in the RNA blot analysis of Tri6 in the non DON-inducing conditions, where expression of Tri6 is elevated even in the Tri6 $\Delta$ mutant strain (lane 2,

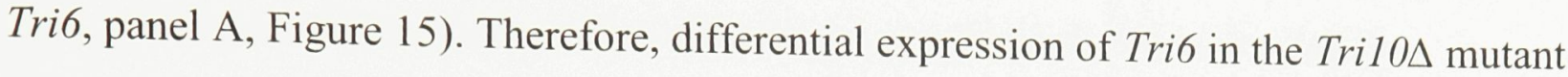
strain could be attributed to the auto-regulation of Tri6 gene, rather than any dependence on Tri10. In a recent study by Seong et al., who performed expression profile analysis in

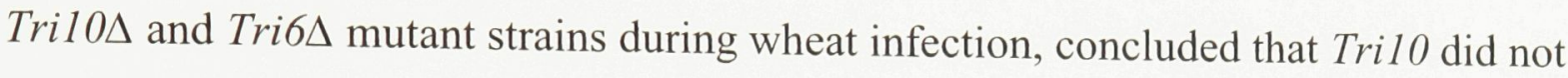
regulate Tri6 since Tri6 expression was not reduced in the Tri10 $\Delta$ strain during plant infection (Seong et al., 2009). Together, this study and Seong et al. (2009) have provided evidence that Tri10 and Tri6 are regulated independently of each other in vitro and in planta.

\subsection{Comparison of in vitro and in planta gene expression profiles}

In the same study performed by Seong et al., a total of 39 genes were down regulated in the Tri10 $\Delta$ mutant strain whereas a total of 224 genes were down regulated in the Tri6 6 mutant strain. Among them, 27 genes were co-regulated by Tri10 and Tri6 including ten Tri genes, Tri1, 3, 4, 5, 8, 11, 12, 14, 15, 101 (Seong et al., 2009). Similar to our observations, they also observed that neither Tril 10 nor Tri6 are regulated by each other. Interestingly, while we observed Tri9 was equally affected in both Tri10 10 and Tri6 6 mutants along with the other ten Tri genes listed in culture conditions, the expression of this gene was only affected in the Tri6 $6 \Delta$ mutant strain during wheat infection only. Since Seong et al. (2009) did not provide the expression level of this gene, 
it is difficult to conclude if the exclusion of Tri9 in their gene expression profile did not meet the two-fold cut-off criteria or that it is indeed not regulated by Trilo.

The genes of the butenolide cluster have been documented to be closely coordinated with the expression of the trichothecene genes (Harris et al., 2007). Our microarray analysis corroborated this observation and indicated that both gene clusters are co-regulated by Tril0 and Tri6. Surprisingly, none of the eight genes in the butenolide cluster was down regulated in Tri10 $\Delta$ and Tri6 $\Delta \Delta$ mutant strains during wheat infection. This in planta result suggests that the expression of this cluster may be related to the type of host that $F$. graminearum infects. Toxic effects of trichothecene have been documented to be host-specific, for instance, non DON-inducing F. graminearum Tri5 mutant strain cannot cause disease in wheat but remains virulent in barley, indicating that while DON acts as a virulence factor on wheat, it is not on barley (Maier et al., 2006).

Among the 97 cytochrome P450s expressed in F. graminearum, six are regulated by both Tri10 and Tri6 in our study, including the three genes (Tri 1, 4,11) involved in the trichothecene biosynthesis pathway. All three trichothecene-associated P450 genes are also regulated by Tri 10 and Tri6 in planta. Of the three other genes coding for P450 monooxygenase regulated in culture, only fg00007 was also down regulated in planta (Seong et al., 2009). As alluded to earlier, host specificity may play a role in the expression of Fusarium genes. This is clearly evident when we compare the number of genes that are affected by these mutant strains. Comparison of in vitro and in planta expression profiles indicated a large discrepancy between the number of genes that are 
differentially regulated in culture by the two mutant strains compared to the genes in the infected wheat heads. There are 960 genes that are down regulated in the Tri $10 \Delta$ mutant in vitro, compared to only 39 genes that are down regulated in planta upon infection with this mutant (Seong et al., 2009). Likewise, the number of down regulated genes in the Tri6 $\Delta$ mutant in vitro is 605 , compared to the 224 down regulated genes in planta (Seong et al., 2009).

\subsection{Roles of Tri10 and Tri6 in secondary metabolism}

One of the major findings of this research is that the regulatory effects imposed by Trilo and Tri6 extend beyond the control of a single secondary metabolite cluster. In addition to the trichothecene biosynthesis pathway, Tril0 and Tri6 also regulate six of the eight genes residing in the butenolide gene cluster on chromosome 2. The seventh gene, fg08078, was down regulated by four-fold in Tri104, but only by 1.8 -fold in Tri64, suggesting that its expression is differentially regulated by Tril0 with respect to our twofold cut-off criteria. Like trichothecene biosynthesis pathway, butenolide production is activated under nutrient limiting conditions, but at an earlier time point (Harris et al., 2007). Although the expression data suggests a direct relationship between these two pathways, the product of each pathway shows an opposite relationship (Harris et al., 2007). In a $F$. graminearum strain that accumulates $15-\mathrm{ADON}$ to high levels, there is a corresponding decrease in butenolide production (Harris et al., 2007). Deletion analysis of $f g 08079$, which codes for a cytochrome $\mathrm{P} 450$, resulted in the loss of butenolide production but not trichothecene synthesis (Harris et al., 2007). Wheat infection assays of fg080794 mutant strain indicated that butenolide production is not required for the infection and spreading of the fungal pathogen in wheat, as the mutant was as virulent as 
wild type (Harris et al., 2007). As mentioned, expression of Tril 5 was negatively affected by exogenous addition of T2-toxin in F. sporotrichioides (Alexander et al., 2004), however, its role in the trichothecene biosynthesis pathway was not conclusive. Given the inverse relationship between the accumulation of 15-ADON and butenolide, it would be interesting to ascertain a role for Tri 15 in the context of butenolide production.

Antibiotic assays of the two mycotoxins butenolide and T2-toxin showed that butenolide at $200 \mu \mathrm{g}$ could inhibit the growth of nine bacteria but had no effect on fungi, whereas T2-toxin at $50 \mu \mathrm{g}$ was toxic to six different fungi but exerted no deleterious effects on bacteria (Burmeister and Hesseltine, 1970). This suggested that Fusarium spp. produces different mycotoxins to eliminate different groups of potential competitors in nature, ultimately promoting its own fitness and survival. In addition to eliminating competitors, butenolide was also demonstrated to act synergistically with another mycotoxin, enniatin, to cause lesions on the leaves of a flowering plant 'spotted knapweed' (Centura maculosa) (Hershenhorn et al., 1992). Fusarium avenaceum produces both butenolide and enniatin to cause necrotic lesions on spotted knapweed, thus promoting its virulence (Hershenhorn et al., 1992). When $5 \mu \mathrm{g}$ of butenolide and 22 $\mu \mathrm{g}$ of enniatin were applied onto detached leaves of spotted knapweed, necrotic lesions similar to those caused by $F$. avenaceum were observed, however, when individually applied onto the leaves, necrotic symptoms were not observed, suggesting that both mycotoxins act synergistically and promote pathogenicity (Hershenhorn et al., 1992). Although a direct role of butenolide in F. graminearum has not been ascertained, it is 
possible that it acts synergistically with other toxins, including trichothecenes to increase fitness through the elimination of competitors.

\subsection{Other candidates of the trichothecene biosynthesis pathway}

As described in Figure 3, a number of genes involved in the biosynthesis of DON have yet to be identified. Our microarray analysis revealed that in addition to regulating the three cytochrome P450s that participate in the trichothecene biosynthesis pathway, Tri10 and Tri6 also regulate another three cytochrome P450s, namely, fg00007, fg08023 and $f g d 224-50$. In the trichothecene biosynthesis pathway, P450 monooxygenases are involved in various hydroxylation steps. As indicated in Figure 14, the synthesis of 7, 8dihydroxycalonectrin is speculated to be catalyzed by two P450s. However, only one, namely Tril, has been identified. It is conceivable that any of the remaining P450s identified in our microarray data could participate in this catalyzation step.

In addition to playing a role in the DON biosynthesis pathway, cytochrome P450s also participate in the detoxification of phytoalexins produced by plants during infection. For example, the fungal pathogen Nectria haematococca (anamorphic state Fusarium solani) produces the enzyme pisatin demethylase, a cytochrome P450 that allows the fungus to demethylate pisatin, a phytoalexin produced by pea plants (Desjardins et al., 1984). The demehtylated pisatin is reduced in toxicity compared to pisatin, therefore allowing the fungus to tolerate and survive through the infection process (Desjardins et al., 1984). Hence, it is worthwhile to investigate the function of these three cytochrome $\mathrm{P} 450$ s, even if they do not participate in the trichothecene biosynthesis pathway. 


\subsection{Differential regulation of genes by Tri10 and Tri6}

Our microarray analysis showed that the total number of genes differentially regulated by Tri10 is 1650 compared to 1089 by Tri6. We observed that $62 \%$ (677) of the genes regulated by Tri6 are also regulated by Tri10. However, a substantial number of genes (60\% or 979 genes) are uniquely affected by Tri 10 alone and similarly $40 \%$ of the genes are uniquely regulated by Tri6. MIPS functional category suggested that while Tri10 and Tri6 seem to commonly regulate genes involved in primary metabolism, Tri10 also appear to exert its influence on genes involved in cellular transport and in defense and virulence. Functional categories of genes assigned by MIPS have overlapping functions and therefore, we cannot conclude that genes regulated by Tri10 or Tri6 are limited to distinct category. Experiments with over-expression of Tril0 in a Tri64 mutant strain and vice versa might tease out genes that are differentially regulated by Trilo and Tri6. 


\section{References}

Abbas H and Boyette C. (1992). Phytotoxicity of Fumonisin $\mathrm{B}_{1}$ on weed and crop species. Weed Technology 6: 548-552.

Alexander N, McCormick S, Holm T. (1999). Tri12, a trichothecene efflux pump from Fusarium sporotrichioides: gene isolation and expression in yeast. Molecular and General Genetics 261: 977-984.

Alexander N, McCormick S, Larson T and Jurgenson J. (2004). Expression of Tril 5 of Fusarium sporotrichioides. Current Genetics 45: 157-162.

ARO: Laboratory for Food and Residue Analyses. (2003). Worldwide regulations for mycotoxins in food and feed in 2003. National Institute for Public Health and the Environment, Netherlands.

Bacon C. (1995). Toxic endophyte-infected tall fescue and range grasses: a historical perspective. Journal of Animal Science 73: 861-870.

Bennett G, Shotwell O and Hesseltine C. (1980). Destruction of Zearalenone in contaminated corn. The Journal of the American Oil Chemists' Society 57: 245-247.

Boutigny A, Richard F and Barreau C. (2008). Natural mechanisms for cereal resistance to the accumulation of Fusarium trichothecenes. European Journal of Plant Pathology 121: $411-423$.

Brayer K and Segal D. (2008). Keep your fingers off my DNA: Protein-protein interactions mediated by $\mathrm{C} 2 \mathrm{H} 2$ zinc finger domains. Cell Biochemistry and Biophysics 50: $111-131$.

Brown D, McCormick S, Alexander N, Proctor R, and Desjardins A. (2001). A genetic and biochemical approach to study trichothecene diversity in Fusarium sporotrichioides and Fusarium graminearum. Fungal Genetics and Biology 32: 121-133.

Brown D, McCormick S, Alexander N, Proctor R and Desjardins A. (2002). Inactivation of a cytochrome P-450 is a determinant of trichothecene diversity in Fusarium species. Fungal Genetics and Biology 36: 224-233.

Brown D, Dyer R, McCormick S, Kendra D and Plattner R. (2004). Functional demarcation of the Fusarium core trichothecene gene cluster. Fungal Genetics and Biology 41: 454-462.

Brown, R. (2005). Zinc finger proteins: getting a grip on RNA. Current Opinion in Structural Biology 15: 94-98. 
Burmeister H and Hesseltine C. (1970). Biological assays for two mycotoxins produced by Fusarium tricinctum. Applied Microbiology 20: 437-440.

Cappellini R and Peterson J. (1965). Macroconidium formation in submerged cultures by a non-sporulating strain of Gibberella zeae. Mycologia 57: 962-966.

Champeil A, Dore T and Fourbet J. (2004). Fusarium head blight: epidemiological origin of the effects of cultural practices on head blight attacks and the production of mycotoxins by Fusarium in wheat grains. Plant Science 166: 1389-1415.

Couture L, Dhont C, Chalifour F, Drapeau R, Tremblay G, Castonguay Y, Belanger G and Nadeau P. (2002). Fsarium root and crown rot in alfalfa subjected to autumn harvests. Canadian Journal of Plant Science 82: 621-624.

De Nijs M, Rombouts F and Notermans S. (1996). Fusarium molds and their mycotoxins. Journal of food safety 16: 15-58.

Desjardins A, Matthews D and VanEtten H. (1984). Solubilization and reconstitution of pisatin demethylase, a cytochrome P-450 from the pathogenic fungus Nectria haematococca. Plant Physiology 75: 611-616.

Desjardins A, Plattner D and Spencer G. (1988). Inhibition of trichothecene toxin biosynthesis by naturally occurring shikimate aromatics. Phytochemistry 27: 767-771.

Desjardins, A. (2006). Fusarium mycotoxins: chemistry, genetics and biology. St Paul, Minnesota: The American Phytopathological Society.

Desjardins A and Proctor R. (2007). Molecular biology of Fusarium mycotoxins. International Journal of Food Microbiology 119: 47-50.

Foroud N and Eudes F. (2009). Trichothecenes in cereal grains. International Journal of Molecular Sciences 10: 147-173.

Gardiner D, Kazan K and Manners J. (2009). Nutrient profiling reveals potent inducers of trichothecene biosynthesis in Fusarium graminearum. Fungal Genetics and Biology 46: 604-613.

Gelderblom W, Kriek N, Marasas W and Thiel P. (1991). Toxicity and carcinogenicity of Fusarium moniliforme metabolite, fumonisins $\mathrm{B}_{1}$ in rats. Carcinogenesis 12: 1247-1251.

Glynn N, Ray R, Edwards S, Hare M, Parry D, Barnett C and Beck J. (2007).

Quantitative Fusarium spp. And Microdochium spp. PCR assays to evaluate seed treatments for the control of Fusarium seedling blight of wheat. Journal of Applied Microbiology 102: 1645-1653. 
Goswami R and Kistler H. (2004) Heading for disaster: Fusarium graminearum on cereal crops. Molecular Plant Pathology 5: 515-525.

Guenther J and Trail F. (2005). The development and differentiation of Gibberella zeae (anamorph: Fusarium graminearum) during colonization of wheat. Mycologia 97: 229237.

Hallen H, Huebner M, Shiu S, Guldener U and Trail F. (2007). Gene expression shifts during perithecium development in Gibberella zeae (anamorph Fusarium graminearum), with particular emphasis on ion transport proteins. Fungal Genetics and Biology 44: 1146-1156.

Harris L, Alexander N, Saparno A, Blackwell B, McCormick S, Desjardins A, Robert L, Tinker N, Hattori J, Piche C, Schernthaner J, Watson R and Ouellet T. (2007). A novel gene cluster in Fusarium graminearum contains a gene that contributes to butenolide synthesis. Fungal Genetics and Biology 44: 293-306.

Hershenhorn J, Park S, Stierle A and Strobel G. (1992). Fusarium avenaceum as a novel pathogen of spotted knapweed and its phytotoxins, acetamido-butenolide and enniatin B. Plant Science 86: 155-160.

Hohn T, Krishna R and Proctor R. (1999). Characterization of a transcriptional activator controlling trichothecene toxin biosynthesis. Fungal Genetics and Biology 26: 224-235.

Hou Z, Xue C, Peng Y, Katan T, Kistler C and Xu J. (2002). A mitogen-activated protein kinase gene (MGVI) in Fusarium graminearum is required for female fertility, heterokaryon formation and plant infection. Molecular Plant-Microbe Interactions 15: 1119-1127.

IARC: International Agnecy for Research on Cancer. (1993). Some naturally occurring substances: food items and constituents, heterocyclic aromatic amines and mycotoxins. Vol 56. World Health Organization. Oxford, England: Oxford University Press.

Iuchi S. (2001). Review: three classes of $\mathrm{C}_{2} \mathrm{H}_{2}$ zinc finger proteins. Cellular and Molecular Life Sciences 58: 625-635.

Keller N and Hohn T. (1997). Review: metabolic pathway gene clusters in filamentous fungi. Fungal Genetics and Biology 21: 17-29.

Khan M, Fischer S, Egan D and Doohan F. (2006). Biological control of Fusarium seedling blight disease of wheat and barley. Phytopathology 96: 386-394.

Kimura M, Kaneko I, Komiyama M, Takatsuki A, Koshinol H, Yoneyama K and Yamaguchi I. (1998). Trichothecene 3-O-Acetyltransferase protects both the producing organism and transformed yeast from related mycotoxins: Cloning and characterization of Tri101. The Journal of Biological Chemistry 273: 1654-1661. 
Kimura M, Tokai T, O’Donnell K, Ward T, Fujimura M, Hamamoto H, Shibata T and Yamaguchi I. (2003). The trichothecene biosynthesis gene cluster of Fusarium graminearum F15 contains a limited number of essential pathway genes and expressed non-essential genes. FEBS Letters 539: 105-110.

Kimura M, Tokai T, Takahashi-Ando N, Ohsato S and Fujimura M. (2007). Molecular and genetic studies of Fusarium trichothecene biosynthesis: pathways, genes and evolution. Bioscience, Biotechnology and Biochemistry 71: 2105-2123.

Kordic B, Pribicevic S, Muntanola-Cvetkovic M, Nicolic P, and Nicolic B. (1992). Experimental studies of the effects of known quantities of zearalenone on swine reproduction. Journal of Environmental Pathology, Toxicology and Oncology 11: 53-55.

Krishna S, Majumdar I, Grishin N. (2003). Survey and summary: structural classification of zinc fingers. Nucleic Acids Research 31: 532-550.

Krizek B, Amann B, Kilfoil V, Merkle D and Berg J. (1991). A consensus zinc finger pepetide: design, high-affinity, metal binding, a $\mathrm{pH}$-dependent structure, and a His to Cys sequence variant. Journal of the American Chemical Society 113: 4518-4523.

Kuiper-Goodman T, Scott P and Watanabe H. (1987). Risk assessment of the mycotoxin zearalenone. Regulatory Toxicology and Pharmacology 7: 253-306.

Lee T, Han YK, Kim KH, Yun SH, and Lee YW. (2002). Tri13 and Tri7 determine deoxynivalenol- and nivalenol- producing chemotypes of Gibberella zeae. Applied and Environmental Microbiology 68: 2148-2154.

Lombaert G, Pellaers P, Orscoe V, Mankotia M, Neil R, Scott P. (2003). Mycotoxins in infant cereal foods from the Canadian retail market. Food Additives and Contaminants 20: 494-504.

Maier F, Miedaner T, Hadeler B, Felk A, Salomon S, Lemmens M, Kassner H and Schafer W. (2006). Involvement of trichothecenes in fusarioses of wheat, barley and maize evaluated by gene disruption of the trichodiene synthase (Tri5) gene in three field isolates of different chemotype and virulence. Molecular Plant Pathology 7: 449-461.

Maniatis T, Fritsch E and Sambrook J. (1989). Molecular Cloning: A Laboratory Manual. New York: Cold Spring Harbor Laboratory Press.

Matsumoto G, Wuchiyama J, Shingu Y, Kimura M, Yoneyama K and Yamaguchi I. (1999). The trichothecene biosynthesis regulatory gene from the type B producer Fusarium strains: sequence of Tri6 and its expression in Escherichia coli. Bioscience, Biotechnology, and Biochemistry 63: 2001-2004. 
McCormick S, Harris L, Alexander N, Ouellet T, Saparno A, Allard S and Desjardins A. (2004). Tril in Fusarium graminearum encodes a P450 oxygenase. Applied and Environmental Microbiology 70: 2044-2051.

Mehrotra R and Aneja K. (1990). An Introduction to Mycology. New Delhi: New Age International Limited.

Michielse C and Rep M. (2009). Pathogen profile update: Fusarium oxysporum. Molecular Plant Pathology 19: 311-324.

Miller J and Arnison P. (1986). Degradation of deoxynivalenol by suspension cultures of the fusarium head blight resistant wheat cultivar Frontana. Canadian Journal of Plant Pathology 8: 147-150.

Mills, J. (1989). Mycotoxins and toxigenic fungi on cereal grains in western Canada. Canadian Journal of Physiology and Pharmacology 68: 982-986.

Namiki F, Matsunaga M, Okuda M, Inoue I, Nishi K, Fujita Y and Tsuge T. (2001). Mutation of an arginine biosynthesis gene causes reduced pathogenicity in Fusarium oxysporum f. sp. melonis. Molecular Plant-Microbe Interactions 14: 580-584.

Nissen P, Hansen J, Ban N, Moore PB and Steitz TA. (2000). The structural basis of ribosome activity in peptide bond synthesis. Science 289: 920-930.

O’Donnell K, Kistler H, Tacke B and Casper H. (2000). Gene genealogies reveal global phylogeographic structure and reproductive isolation among lineages of Fusarium graminearum, the fungus causing wheat scab. Proceedings of the National Academy of Sciences of the United States of America 97: 7905-7910.

O'Donnell K, Ward T, Geiser D, Kistler H and Aoki T. (2004). Genealogical concordance between the mating type locus and seven other nuclear genes supports formal recognition of nine phylogenetically distinct species within the Fusarium graminearum clade. Fungal Genetics and Biology 41: 600-623.

Oshlack A, Emslie D, Corcoran L and Smyth G. (2007). Normalization of boutique twocolor microarrays with a high proportion of differentially expressed probes. Genome Biology 8: R2.

Parry D. (1990). Plant pathology in agriculture. New York: Cambridge University Press.

Parry D, Jenkinson P, and McLeod L. (1995). Fusarium ear blight (scab) in small grain cereals - a review. Plant Pathology 44: 207-238.

Peplow W, Tag A, Garifullina G and Beremand M. (2003). Identification of new genes positively regulated by Tri10 and a regulatory network for trichothecene mycotoxin production. Applied and Environmental Microbiology 69: 2731-2736. 
Pereira C, Chen YH and McDonald F. (2009). Total synthesis of sphingolipid biosynthesis inhibitor Fumonisin $\mathrm{B}_{1}$. Journal of American Chemical Society 131: 6066-
6067 .

Pestka J, El-Bahrawy A and Hart P. (1985). Deoxynivalenol and 15-monoacetyl deoxynivalenol production by Fusarium graminearum R6576 in liquid media. Mycopathologia 91:23-28.

Pestka J and Smolinski A. (2005). Deoxynivalenol: toxicology and potential effects on humans. Journal of Toxicology and Environmental Health, Part B 8: 39-69.

Pitt J. (2000). Toxigenic fungi: which are important? Medical Mycology 38: 17-22.

Poppenberger B, Berthiller F, Lucyshyn D, Sieberer T, Schuhmacher R, Krska R, Kuchler K, Glossl J, Luschnig C and Adam G. (2003). Detoxification of the Fusarium mycotoxin deoxynivalenol by a UDP-glycosyltransferase from Arabidopsis thaliana. The Journal of Biological Chemistry 278: 47905-47914.

Proctor R, Hohn T, McCormick S and Desjardins A. (1995). Tri6 encodes an unusual zinc finger protein involved in regulation of trichothecene biosynthesis in Fusarium sporotrichioides. Applied and Environmental Microbiology 61: 1923-1930.

Robson G, Wiebe M, Cunliffe B and Trinci A. (1995). Choline- and acetylcholineinduced changes in the morphology of Fusarium graminearum: evidence for the involvement of choline transport system and acetylcholinesterase. Microbiology 141: 1309-1314.

Schroeder HW and Christensen JJ. (1963). Factors affecting the resistance of wheat to scab caused by Gibberella zeae. Phytopathology 53: 831-818.

Seong KY, Pasquali M, Zhou X, Song J, Hilburn K, McCormick S, Dong Y, Xu JR and C Kistler. (2009) Global gene regulation by Fusarium transcription factors Tri6 and Tri10 reveals adaptations for toxin biosynthesis. Molecular Microbiology 72: 354-367.

Shimada T and Otani M. (1990). Effects of Fusarium mycotoxins on the growth of shoots and roots at germination in some Japanese wheat cultivars. Cereal Research Communications 18: 229-232.

Stack R. (1989). A comparison of the inoculum potential of ascospores and conidia of Gibberella zeae. Canadian Journal of Plant Pathology 11: 137-142.

Sudakin D. (2003). Trichothecenes in the environment: relevance to human health. Toxicology Letters 143: 97-107. 

Suzuki M and Muranaka T. (2007). Molecular genetics of plant sterol backbone
synthesis. Lipids 42: 47-54.

Tag A, Garifullina G, Peplow A, Ake C, Phillips T, Hohn T and Beremand M. (2001). A novel regulatory gene, Tri10, controls trichothecene toxin production and gene expression. Applied and Environmental Microbiology 67: 5294-5302.

Taylor J, Jacobson D, Kroken S, Kasuga T, Geiser D, Hibbett D and Fisher M. (2000). Phylogenetic species recognition and species concepts in Fungi. Fungal Genetics and Biology 31: 21-32.

Taylor R, Saparno A, Blackwell B, Anoop V, Gleddie S, Tinker N and Harris L. (2008). Proteomic analysis of Fusarium graminearum grown under mycotoxin-inducing conditions. Proteomics 8: 2256-2265.

Trail F, Xu H, Loranger R and Gadoury D. (2002). Physiological and environmental aspects of ascospore discharge in Gibberella zeae (anamorph Fusarium graminearum). Mycologia 94: 181-189.

Trail F, Gaffoor I and Vogel S. (2005). Ejection mechanics and trajectory of the ascospores of Gibberella zeae (anamorph Fusarium graminearum). Fungal Genetics and Biology 42: 528-533.

Trail, F. (2009). For blighted waves of grain: Fusarium graminearum in the postgenomics era. Plant Physiology 149: 103-110.

Urlacher V and Eiben S. (2006). Cytochrome P450 monooxygenases: perspectives for synthetic application. Trends in Biotechnology 24: 324-330.

Wiebe M, Novakova M, Miller L, Blakebrough M, Robson G, Punt P and Trinci A. (1997). Protoplast production and transformation of morphological mutants of the Quorn myco-protein fungus, Fusarium graminearum A3/5, using the hygromycin B resistance plasmid pAN7-1. Mycological Research 101: 871-877.

Windels, C. (2000). Economics and social impacts of Fusarium Head Blight: Changing farms and rural communities in the Northern Great Plains. Phytopathology 1: 17-21.

Yazar S and Omurtag G. (2008). Fumonisins, Trichothecenes and Zearalenone in cereals. International Journal of Molecular Sciences 9: 2062-2090. 
Appendix

Table A1: Primer Sequences used for Creating Tri10 Disruption Construct

\begin{tabular}{cl}
\hline Primer & \multicolumn{1}{c}{ Sequence $\mathbf{5}^{\prime} \rightarrow \mathbf{3}^{\prime}$} \\
\hline P1 & GACGCTTGCATATTCAGGC \\
P2 & GATGTCAGGCCATTTTCACCGATACCCGAAGTCTTTC \\
P3 & GAAAGACTTCGGGTATCGGTGAAAATGGCCTGACATC \\
P4 & CACTCGTCTAAGCAGATGG \\
P5 & TGAAAATGGCCTGACATCAT \\
P6 & AGCTCTTGTTCGGTCGGCATCTAC \\
\hline
\end{tabular}

Table A2: Primer Sequences used for Creating Tri6 Disruption Construct

\begin{tabular}{cl}
\hline Primer & \multicolumn{1}{c}{ Sequence 5, $\boldsymbol{\rightarrow}$ 3, } \\
\hline P1 & GTCCTCAGTATCGCCGTGTCAG \\
P2 & GATGTCAGGCCATTTTCAGAGAGTATGTGGGGAAGTTGTCAAAG \\
P3 & TGCCGACCGAACAAGAGCTGACAGTGCGGTGCCCTTGG \\
P4 & GCCAATGCCAGCAAAAAGTATC \\
P5 & CTTTGACAACTTCCCCACATACTCTCTGAAAATGGCCTGACATC \\
P6 & CCAAGGGCACCGCACTGTCAGCTCTTGTTCGGTCGGCA \\
\hline
\end{tabular}

Table A3: Primer Sequences used for Creating Tri6 and Tri10 Addback Constructs

\begin{tabular}{cl}
\hline Primer & \multicolumn{1}{c}{ Sequence $\mathbf{5}^{\prime} \boldsymbol{\rightarrow}$; } \\
\hline Tri10 AB F & GACGCTTGCATATTCAGGC \\
Tri10AB R & CACTCGTCTAAGCAGATGG \\
Tri6 AB F & GTCCTCAGTATCGCCGTGTCAG \\
Tri6 AB R & GCCAATGCCAGCAAAAAGTATC \\
\hline
\end{tabular}

Table A4: Primers used for confirmation Tri104 and Tri64 Deleted Mutant Strains

\begin{tabular}{llc}
\hline \multicolumn{1}{c}{ Primer } & \multicolumn{1}{c}{ Sequence 5' $\rightarrow$ 3' } & Gene \\
\hline Tri10 orf F & GAATTCTAGTCATCATGGATTTCC & Trilo \\
Tri10 orf R & GAATTCATCCCTACCAAAGTAGCACT & Trilo \\
Hyg P5 & TGAAAATGGCCTGACATCAT & Hygromycin \\
Hyg P6 & AGCTCTTGTTCGGTCGGCATCTAC & Hygromycin \\
Geneticin ENTR & CACCATGCCAGTTGTTCCCAG & Geneticin \\
Geneticin Down & CATATCGATCTTGGGTAGAAT & Geneticin \\
T6 Fsp & CATGCCAAGGACTTTGTCCC & Tri6 \\
T6 End R & GTGTATCCGCCTATAGTGAT & Tri6 \\
\hline
\end{tabular}


Table A5: Primers used to Amplify Non-Radiolabeled Probe Templates

\begin{tabular}{llc}
\multicolumn{1}{c}{ Primer } & \multicolumn{1}{c}{ Sequence 5 $^{\rightarrow} \rightarrow$ 3 $^{\prime}$} & Gene \\
\hline Tri1 RT F & GTATCTGTCCACACCACCACC & Tril \\
Tri1 RT R & GTCATCCTGTACCAATTCC & Tril \\
Fg07 RT F & CCCAGAGGTCCTCAAGAAAGCCC & Fg00007 \\
Fg07 RT R & CAGTACGACCATATCCTGCTGG & Fg00007 \\
T6 Fsp & CATGCCAAGGACTTTGTCCC & Tri6 \\
T6 End R & GTGTATCCGCCTATAGTGAT & Tri6 \\
Tri10 orf F & GAATTCTAGTCATCATGGATTTCC & Trilo \\
Tri10 orf R & GAATTCATCCCTACCAAAGTAGCACT & Trilo \\
Hyg P5 & TGAAAATGGCCTGACATCAT & Hygromycin \\
Hyg P6 & AGCTCTTGTTCGGTCGGCATCTAC & Hygromycin \\
\hline
\end{tabular}

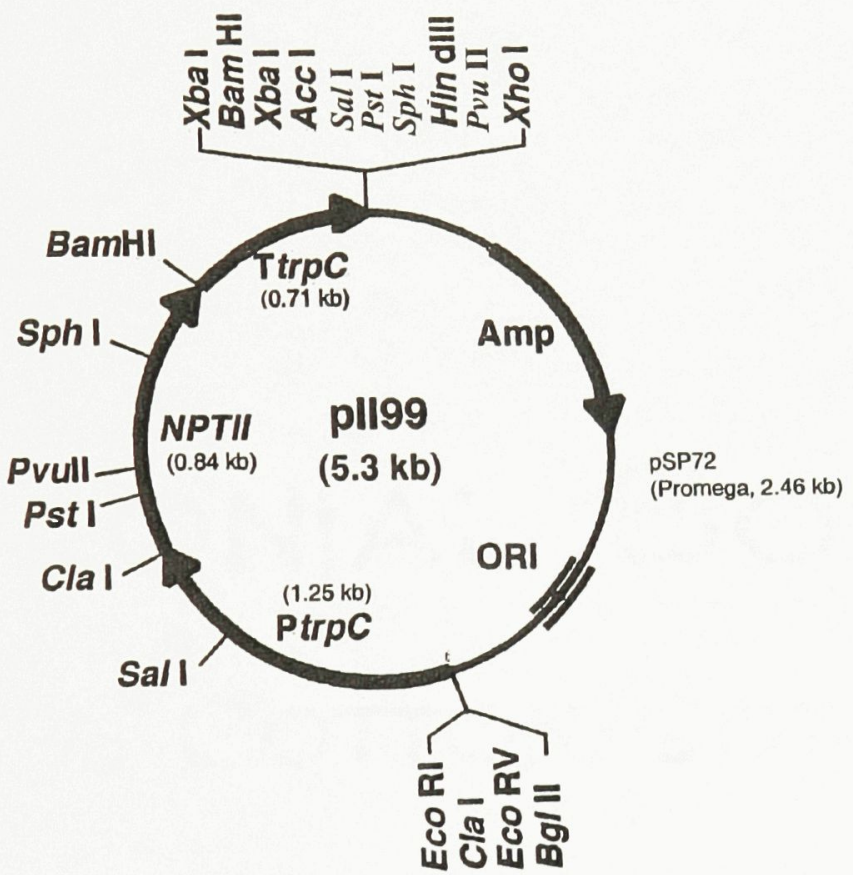

Figure A1: pII99 Vector Map (Namiki et al., 2001) 


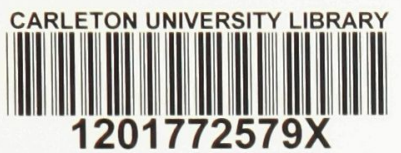

Differential roles of TRI10 and TRI6 in Fusarium graminearum

THS

M.SC.

2009

L48

DISC 